Pure and Applied Mathematics Quarterly

Volume 4, Number 2

(Special Issue: In honor of

Fedor Bogomolov, Part 1 of 2$)$

$237-318,2008$

\title{
A Degree Doubling Formula for Braid Monodromies and Lefschetz Pencils
}

\author{
Denis Auroux ${ }^{1}$ and Ludmil Katzarkov ${ }^{2}$
}

Contents

1. Introduction 238

1.1. Braid monodromy invariants 240

1.2. The degree doubling process 244

1.3. Degree doubling for symplectic Lefschetz pencils 248

2. Stably quasiholomorphic coverings 249

2.1. Quasiholomorphic coverings and braided curves 249

2.2. Stably quasiholomorphic coverings 251

2.3. Proof of Proposition $1 \quad 260$

3. The degree doubling formula for braid monodromies 264

3.1. Generalities about the braid group 264

3.2. The folding process 266

3.3. The $V_{2}$ branch curve 271

Received February 27, 2006.

${ }^{1}$ Partially supported by a Sloan research fellowship and NSF Grant DMS-0244844.

${ }^{2}$ Partially supported by a Sloan research fellowship and NSF Grant DMS-9875383. 
3.4. Regeneration of the mutual intersections 275

3.5. The assembling rule 284

3.6. The degree doubling formula 292

4. The degree doubling formula for Lefschetz pencils 305

4.1. Braid groups and mapping class groups 305

4.2. The degree doubling formula for Lefschetz pencils 309

$\begin{array}{ll}\text { References } & 317\end{array}$

\section{INTROduCtion}

It was shown in [1] that every compact symplectic 4 -manifold $(X, \omega)$ can be realized as an approximately holomorphic branched covering of $\mathbb{C P}^{2}$ whose branch curve is a symplectic curve in $\mathbb{C P}^{2}$ with cusps and nodes as only singularities (however the nodes may have reversed orientation). Such a covering is obtained by constructing a suitable triple of sections of the line bundle $L^{\otimes k}$, where $L$ is a line bundle obtained from the symplectic form (its Chern class is given by $c_{1}(L)=\frac{1}{2 \pi}[\omega]$ when this class is integral), and where $k$ is a large enough integer. Moreover, it was shown in [5] that the braid monodromy techniques introduced by Moishezon and Teicher in algebraic geometry (see e.g. [12, 14, 20]) can be used in this situation to derive, for each large enough value of the degree $k$, monodromy invariants which completely describe the symplectic 4-manifold $(X, \omega)$ up to symplectomorphism. These invariants are also related to those constructed by Donaldson and arising from the monodromy of symplectic Lefschetz pencils [9], which also are defined only for large values of $k$.

The monodromy invariants arising from branched coverings or symplectic Lefschetz pencils give, in principle at least, complete information about the topology of a symplectic manifold. Given a suitable way to extract numerical invariants from them, one can hope to use them to symplectically tell apart certain pairs of mutually homeomorphic algebraic surfaces of general type, such as the Horikawa manifolds, which no currently available symplectic invariant can distinguish. However, their practical usefulness is immensely limited by the difficulties 
involved in their calculation, even though the computations by Moishezon, Teicher and Robb of the braid monodromies for certain simple types of algebraic surfaces $\left(\mathbb{C P}^{2}, \mathbb{C P}^{1} \times \mathbb{C P}^{1}\right.$, complete intersections) $[20]$ give some reason to be hopeful (see also [4] for other examples). Moreover, the difficulty of comparing two braid group or mapping class group factorizations up to Hurwitz equivalence is a major obstacle. In fact, the general Hurwitz problem in braid groups is known to be undecidable [11]; however the argument does not seem to apply to the specific case where the factors are powers of half-twists.

One of the main technical problems arising in this program is that the monodromy only becomes a symplectic invariant when the degree is large enough, which makes it necessary to handle whole sequences of braid factorizations. Even when the entire sequence can be obtained directly out of Moishezon-Teicher style calculations $[20,4]$, it is very difficult to understand how to extract meaningful information out of the monodromy data, due to the lack of a clear relationship between the monodromies arising for different values of the twisting parameter $k$.

The aim of this paper is to describe an explicit formula relating the braid monodromy invariants obtained for a given degree $k$ to those obtained for the degree $2 k$. The interest of such a formula is obvious from the above considerations, especially as direct computations of braid monodromy often become intractable for large degrees. We also give a similar formula for the monodromy of symplectic Lefschetz pencils; this formula, which may have even more applications than that for braid monodromies, answers a question first considered by Donaldson and for which a partial (non-explicit) result has been obtained by Smith [19]. Although the formula for pencils is much simpler than that for branched coverings, the currently available technology for monodromy calculations seems insufficient to allow a direct proof.

The techniques introduced in this paper suggest a wide range of applications. First of all, calculations similar to those in this paper appear in any situation involving iterated branched coverings ; for example, the invariants defined by Moishezon and Teicher should become effectively computable for a much larger class of algebraic surfaces (see e.g. $\S 7$ of [4]).

An obvious class of applications is to study the properties of high-degree branched coverings or Lefschetz pencils. For example, Smith has shown using 
a degree doubling argument that any compact symplectic 4-manifold admits a symplectic Lefschetz pencil without reducible singular fibers [19]. Although this specific result can be obtained just from the universality property of degree doubling rather than from the actual formula, other applications require a more detailed understanding of the degree doubling process.

More importantly, the degree doubling formula provides precise information on the behavior of various monodromy-related invariants as the parameter $k$ increases. For example, it is in principle possible to describe how the fundamental group of the complement of the branch curve, or more generally any other invariant directly related to the monodromy group of the branched covering or Lefschetz pencil, depends on the parameter $k$. It is likely that the conjectures formulated in [4] can be approached from this perspective.

In a similar direction, it is reasonable to expect the degree doubling formula to yield a stability result for the "directed Fukaya categories" introduced by Seidel as invariants of Lefschetz pencils [17]. Unlike the direct calculation methods following Moishezon and Teicher, our formula makes it immediately apparent how Lagrangian spheres lying in standard position inside the degree $k$ pencil automatically lie inside the degree $2 k$ pencil; the explicit description of the additional vanishing cycles makes it possible to hope that, under certain assumptions, the degree $2 k$ pencil can be shown to contain no other such spheres.

Yet another question to which our result may give an answer is that of whether every branched covering over $\mathbb{C P}^{2}$ (or every symplectic Lefschetz pencil) is "of Donaldson type" (see the remark at the end of $\S 1.2$ ).

Finally, extensions to higher-dimensional settings of the stabilization procedure described here are theoretically possible, even though it remains uncertain whether it is actually possible to carry out the calculations.

The remainder of this section is devoted to an overview of braid monodromy invariants $(\S 1.1)$, followed by a sketch of our approach to the degree doubling process and a statement of the main results ( $§ 1.2$ and $\S 1.3)$.

1.1. Braid monodromy invariants. We start by recalling the notations and results (see [5] or [2] for details). Let $f: X \rightarrow \mathbb{C P}^{2}$ be an approximately holomorphic branched covering map as in [1] and [5] : its topology is mostly described by 
that of the branch curve $D \subset \mathbb{C P}^{2}$, which is symplectic and approximately holomorphic. The only singularities of $D$ are double points (with either orientation) and cusps (with the complex orientation only) ; the branching is of order 2 at every smooth point of $D$. Fix a generic projection $\pi: \mathbb{C P}^{2}-\{p t\} \rightarrow \mathbb{C P}^{1}$ whose pole does not belong to $D$. We can assume that $D$ is transverse to the fibers of $\pi$ everywhere except at a finite set of non-degenerate tangency points, where a local model is $x^{2}=y$ with projection to the $x$ component ; moreover, we can also assume that all the special points of $D$ (tangencies and singular points) lie in distinct fibers of $\pi$, and that none of them lies in the fiber above the point at infinity in $\mathbb{C P}^{1}$.

The idea introduced by Moishezon in the case of a complex curve is that, restricting oneself to the preimage of the affine subset $\mathbb{C} \subset \mathbb{C P}^{1}$, the monodromy of $\pi_{\mid D}$ around its critical levels can be used to define a map from $\pi_{1}(\mathbb{C}-$ crit $)$ with values in the braid group $B_{d}$ on $d=\operatorname{deg} D$ strings, called braid monodromy (see e.g. [12]) ; this monodromy is encoded by a factorization of the central element $\Delta_{d}^{2}$ of the braid group $B_{d}$. Namely, the monodromy around the point at infinity in $\mathbb{C P}^{1}$, which is given by the central braid $\Delta_{d}^{2}$, decomposes as the product of the monodromies around the critical levels of the projection to $\mathbb{C P}^{1}$, each of these being conjugate to a power of a half-twist. This construction naturally depends on the choice of an ordered set of generating loops for the free group $\pi_{1}(\mathbb{C}-$ crit $)$.

The same techniques extend almost immediately to the symplectic setting, and the resulting braid factorizations are of the form

$$
\Delta_{d}^{2}=\prod_{j}\left(Q_{j}^{-1} X_{1}^{r_{j}} Q_{j}\right)
$$

where $X_{1}$ is the first standard generator of $B_{d}$ (a positive half-twist), $Q_{j}$ are arbitrary braids and $r_{j} \in\{-2,1,2,3\}$.

The case $r_{j}=1$ corresponds to a tangency point, where the curve $D$ is smooth and tangent to the fiber of the projection $\pi$; the case $r_{j}=2$ corresponds to a nodal point of $D$; the case $r_{j}=-2$ is the mirror image of the previous one, and corresponds to a negative self-intersection of $D$ (this is the only type of point which does not occur in the algebraic case) ; and finally the case $r_{j}=3$ corresponds to a cusp singularity of $D$. 
The above-described braid factorization completely determines the topology of the curve $D$. However it is well-defined only up to the following two algebraic operations. A Hurwitz move is the replacement of two consecutive factors $A$ and $B$ by $A B A^{-1}$ and $A$ respectively (we will say that the factor $A$ has been "moved to the right"; the opposite move, which amounts to replacing $A$ and $B$ by $B$ and $B^{-1} A B$ respectively, will be referred to as "moving $B$ to the left"). Another possibility is global conjugation, i.e. conjugating all factors simultaneously by a given braid. A Hurwitz move amounts to an elementary change in the choice of generating loops for the free group $\pi_{1}(\mathbb{C}-$ crit $)$, while a global conjugation amounts to a change of trivialization of the reference fiber of $\pi_{\mid D}$. Two factorizations represent the same curve $D$ if and only if they are Hurwitz and conjugation equivalent.

To recover a map $X \rightarrow \mathbb{C P}^{2}$ from the monodromy invariants we also need a geometric monodromy representation. Let $D \subset \mathbb{C P}^{2}$ be a curve of degree $d$ with cusps and nodes (possibly negative), and let $\mathbb{C} \subset \mathbb{C P}^{2}$ be a fiber of the projection $\pi: \mathbb{C P}^{2}-\{p t\} \rightarrow \mathbb{C P}^{1}$ which intersects $D$ in $d$ distinct points $q_{1}, \ldots, q_{d}$. Then, the inclusion of $\mathbb{C}-\left\{q_{1}, \ldots, q_{d}\right\}$ into $\mathbb{C P}^{2}-D$ induces a surjective homomorphism on the fundamental groups. Small loops $\gamma_{1}, \ldots, \gamma_{d}$ around $q_{1}, \ldots, q_{d}$ in $\mathbb{C}$ generate $\pi_{1}\left(\mathbb{C P}^{2}-D\right)$, with relations coming from the cusps, nodes and tangency points of $D$. These $d$ loops will be called geometric generators of $\pi_{1}\left(\mathbb{C P}^{2}-D\right)$.

Recall that there exists a natural right action of $B_{d}$ on the free group $F_{d}=$ $\pi_{1}\left(\mathbb{C}-\left\{q_{1}, \ldots, q_{d}\right\}\right) ;$ denote this action by $*$, and recall the following definition $[14]:$

Definition 1. A geometric monodromy representation associated to a curve $D \subset$ $\mathbb{C P}^{2}$ is a surjective group homomorphism $\theta$ from the free group $F_{d}=\pi_{1}(\mathbb{C}-$ $\left.\left\{q_{1}, \ldots, q_{d}\right\}\right)$ to the symmetric group $S_{n}$ of order $n$, such that the $\theta\left(\gamma_{i}\right)$ are transpositions (thus also the $\theta\left(\gamma_{i} * Q_{j}\right)$ ) and

$$
\begin{aligned}
& \theta\left(\gamma_{1} \ldots \gamma_{d}\right)=1, \\
& \theta\left(\gamma_{1} * Q_{j}\right)=\theta\left(\gamma_{2} * Q_{j}\right) \text { if } r_{j}=1, \\
& \theta\left(\gamma_{1} * Q_{j}\right) \text { and } \theta\left(\gamma_{2} * Q_{j}\right) \text { are distinct and commute if } r_{j}= \pm 2, \\
& \theta\left(\gamma_{1} * Q_{j}\right) \text { and } \theta\left(\gamma_{2} * Q_{j}\right) \text { do not commute if } r_{j}=3 .
\end{aligned}
$$


In this definition, $n$ corresponds to the number of sheets of the covering $X \rightarrow \mathbb{C P}^{2}$; the various conditions imposed on $\theta\left(\gamma_{i} * Q_{j}\right)$ express the natural requirements that the map $\theta: F_{d} \rightarrow S_{n}$ should factor through the group $\pi_{1}\left(\mathbb{C P}^{2}-D\right)$ and that the branching phenomena should occur in disjoint sheets of the covering for a node and in adjacent sheets for a cusp. The surjectivity of $\theta$ corresponds to the connectedness of the 4-manifold $X$; more precisely, the image of $\theta$ is a subgroup of $S_{n}$ generated by transpositions and acting transitively on $\{1, \ldots, n\}$, which implies surjectivity.

Operations such as Hurwitz moves and global conjugations should be considered simultaneously on the level of braid factorizations and on that of the corresponding geometric monodromy representations : a Hurwitz move does not affect the geometric monodromy representation, but when performing a global conjugation by a braid $Q$ it is necessary to compose $\theta$ with the automorphism of $F_{d}$ induced by $Q$.

In the symplectic case the curve $D$ can have negative nodes, and as a consequence the uniqueness result obtained in [1] only holds up to cancellation of pairs of nodes. An additional possibility is therefore a pair cancellation move in the braid factorization, where two consecutive factors which are the exact inverse of each other are removed from the factorization. The converse move (a pair creation) is also allowed, but only when it is compatible with the geometric monodromy representation : adding $\left(Q^{-1} X_{1}^{-2} Q\right) \cdot\left(Q^{-1} X_{1}^{2} Q\right)$ somewhere in the braid factorization is only legal if $\theta\left(\gamma_{1} * Q\right)$ and $\theta\left(\gamma_{2} * Q\right)$ are commuting disjoint transpositions.

Definition 2. Two braid factorizations (and the corresponding geometric monodromy representations) are m-equivalent if there exists a sequence of operations which turn one into the other, each operation being either a global conjugation, a Hurwitz move, or a pair cancellation or creation.

We now summarize the main results of [5] :

Theorem 1 ([5]). The compact symplectic 4-manifold $X$ is uniquely characterized by the sequence of braid factorizations and geometric monodromy representations corresponding to the approximately holomorphic coverings of $\mathbb{C P}^{2}$ canonically obtained from sections of $L^{\otimes k}$ for $k \gg 0$, up to m-equivalence. 
It was also shown in [5] that conversely, given a (cuspidal negative) braid factorization and a geometric monodromy representation, one can recover in a canonical way a symplectic 4-manifold (up to symplectomorphism).

1.2. The degree doubling process. We now turn to the topic at hand, namely the phenomena that occur when the degree $k$ is changed to $2 k$.

In all the following, we will assume that $k$ is large enough for the uniqueness properties of Theorem 1 to hold (if the considered coverings happen to be algebraic this assumption is unnecessary). This makes it possible to choose the most convenient process for constructing the branch curve for degree $2 k$ while ensuring that the resulting branch curve is indeed equivalent to the canonical one. As observed in [5], one especially interesting way to obtain the covering map $f_{2 k}: X \rightarrow \mathbb{C P}^{2}$ is to start with the covering map $f_{k}: X \rightarrow \mathbb{C P}^{2}$ and compose it with the Veronese covering $V_{2}: \mathbb{C P}^{2} \rightarrow \mathbb{C P}^{2}$ given by three generic homogeneous polynomials of degree 2 (this is a 4:1 covering whose branch curve has degree 6 , see below). The map $V_{2} \circ f_{k}$ is clearly an approximately holomorphic covering given by sections of $L^{\otimes 2 k}$, and its branch curve is the union of the image by $V_{2}$ of the branch curve $D_{k}$ of $f_{k}$ and $n=\operatorname{deg} f_{k}$ copies of the branch curve $C_{2}$ of $V_{2}$ (the branch curve $C_{2}$ is present with multiplicity $n$ because branching occurs at every preimage by $f_{k}$ of a branch point of $\left.V_{2}\right)$. However at every point where $V_{2}\left(D_{k}\right)$ intersects $C_{2}$ the map $V_{2} \circ f_{k}$ presents a non-generic singular behavior : e.g., composing the branched coverings $(x, y) \mapsto\left(x^{2}, y\right)$ and $(x, y) \mapsto\left(x, y^{2}\right)$ yields the singular map $(x, y) \mapsto\left(x^{2}, y^{2}\right)$, which needs to be perturbed in order to obtain a generic behavior. Further small perturbations are required in order to separate the multiple copies of $C_{2}$; nevertheless, $f_{2 k}$ is obtained as a small perturbation of $V_{2} \circ f_{k}$ and its branch curve $D_{2 k}$ is obtained as a small perturbation of $V_{2}\left(D_{k}\right) \cup n C_{2}$.

For all large enough values of $k$, the approximate holomorphicity and transversality properties of the above-described perturbation of $V_{2} \circ f_{k}$ make it subject to the uniqueness results in [1] and [5] : the coverings constructed directly and those obtained by composition with $V_{2}$ and perturbation therefore become isotopic. So, for all large values of $k$ we can indeed hope to compute the braid factorization of $f_{2 k}$ by this method. 
Also observe that a generic isotopy (1-parameter deformation family) of the curve $D_{k}$ behaves "nicely" with respect to the chosen Veronese covering $V_{2}$, and therefore yields a generic isotopy of the curve $V_{2}\left(D_{k}\right)$. Since generic isotopies do not modify braid factorizations (up to Hurwitz and conjugation equivalences in the algebraic category, or up to $m$-equivalence in the symplectic category), we are allowed to perform a generic isotopy on the curve $D_{k}$ to place it in the most convenient position with respect to the ramification curve of $V_{2}$, and this will not affect the end result.

An important consequence of this observation is that the $k \rightarrow 2 k$ formula we are looking for is universal in the sense that it does not depend on the branch curve $D_{k}$ itself but only on its degree $d$ and on the degree $n$ of the covering $f_{k}$. Indeed, an isotopy can be used to make sure that all the special points of $D_{k}$ (cusps, nodes and tangencies) lie in a small ball $B \subset \mathbb{C P}^{2}$ located far away from $V_{2}^{-1}\left(C_{2}\right)$, and that $D_{k}$ looks like a union of $d$ lines outside of the ball $B$. For example, we can take $V_{2}$ to be a small perturbation of the non-generic quadratic map $V_{2}^{0}:(x: y: z) \mapsto\left(x^{2}: y^{2}: z^{2}\right)$, for which the ramification curve consists of three lines (the coordinate axes), and we can use a linear transformation to contract all the special points of $D$ (tangencies, nodes, cusps) into an arbitrarily small ball $B$ centered at the point $(1: 1: 1)$.

With this setup, the contribution of $D_{2 k} \cap V_{2}(B)$ to the braid monodromy is the same as that of $D_{k} \cap B$, and the braid monodromy coming from $D_{2 k} \cap\left(\mathbb{C P}^{2}-\right.$ $V_{2}(B)$ ) does not depend on the curve $D_{k}$ but only on its degree and on the geometric monodromy representation $\theta$. The braid factorization corresponding to $f_{2 k}$ is therefore of the form

$$
F_{k} \cdot \mathbf{U}_{d, n, \theta},
$$

where $F_{k}$ is the braid factorization for $f_{k}$ (after a suitable embedding of $B_{d}$ into the larger braid group $B_{\bar{d}}$ corresponding to $\left.D_{2 k}\right)$ and $\mathbf{U}_{d, n, \theta}$ is a word in $B_{\bar{d}}$ depending only on $d, n$ and $\theta\left(\bar{d}=2 d+6 n=\operatorname{deg} D_{2 k}\right)$.

From the above considerations, the strategy for obtaining the formula giving the braid factorization for $D_{2 k}$ in terms of the braid factorization for $D_{k}$ is the following. First one needs to understand the braid factorizations corresponding to the two curves $V_{2}\left(D_{k}\right)$ and $C_{2}$ taken separately. More specifically, the braid factorization for $V_{2}\left(D_{k}\right)$ is obtained from that for $D_{k}$ via a "folding" formula describing the effect of the quadratic map $V_{2}$; the braid factorization for $C_{2}$ (and 
consequently for $n$ copies of $C_{2}$ ) is obtained by degenerating it to the branch curve of the map $V_{2}^{0}$, which consists of three lines forming a triangle, giving rise to three similar-looking contributions from their mutual intersections. Next, one has to study the phenomena that arise near the intersections of $C_{2}$ with $V_{2}\left(D_{k}\right)$; these again give rise to three similar contributions (one for each line in the branch curve of $V_{2}^{0}$ ). Finally more calculations are required in order to combine these ingredients into a formula for $D_{2 k}$. The main result is the following (see $\S 3$ for notations):

Theorem 2. Let $f_{k}:\left(X^{4}, \omega\right) \rightarrow \mathbb{C P}^{2}$ be an approximately holomorphic branched covering given by three sections of $L^{\otimes k}$. Denote by $D_{k}$ the branch curve of $f_{k}$, and let $d=\operatorname{deg} D_{k}$ and $n=\operatorname{deg} f_{k}$. Denote by $F_{k}$ the braid factorization corresponding to $D_{k}$, and assume that $d \leq n(n-1)$. Then, with the notations of $\S 3$, the braid factorization corresponding to the branch curve $D_{2 k}$ of $f_{2 k}$ is given up to $m$-equivalence by a formula of the following type, provided that $k$ is large enough:

$$
\Delta_{2 d+6 n}^{2}=\mathbf{T}_{d} \cdot \iota\left(F_{k}\right) \cdot \mathbf{I}_{d, n, \theta}^{\alpha} \cdot \mathbf{I}_{d, n, \theta}^{\beta} \cdot \mathbf{V}_{n}^{\alpha \beta} \cdot \mathbf{V}_{n}^{\alpha \gamma} \cdot \mathbf{V}_{n}^{\beta \gamma} \cdot \mathbf{I}_{d, n, \theta}^{\gamma} \cdot
$$

In this formula, $F_{k}$ is viewed as a factorization in $B_{2 d+6 n}$ using a certain natural embedding $\iota: B_{d} \hookrightarrow B_{2 d+6 n}$. The other terms correspond to universal contributions (depending only on $d, n$ and $\theta$ ): more precisely, $\mathbf{T}_{d}$ arises from the folding of $D_{k}$ by the quadratic map $V_{2}$, while $\mathbf{V}_{n}^{\alpha \beta}, \mathbf{V}_{n}^{\alpha \gamma}, \mathbf{V}_{n}^{\beta \gamma}$ arise from the braid monodromy of $n$ parallel copies of the curve $C_{2}$, and $\mathbf{I}_{d, n, \theta}^{\alpha}, \mathbf{I}_{d, n, \theta}^{\beta}, \mathbf{I}_{d, n, \theta}^{\gamma}$ correspond to the intersections of $V_{2}\left(D_{k}\right)$ with $C_{2}$. The individual factors in each of these expressions are described in $\S 3.6$. The asymmetry in the ordering of terms in (1) is mostly a consequence of choices made to keep these individual factors as simple as possible, but can ultimately be traced to the choice of a specific projection from $\mathbb{C P}^{2}$ to $\mathbb{C P}^{1}$.

The proof of Theorem 2 is carried out in Sections 2 and 3 of this paper: the strategy of proof outlined above is carefully justified in $\S 2$ (cf. in particular Propositions 1 and 2); general properties of the braid group and notations are introduced in $\S 3.1 ; \S 3.2$ describes the folding formula which gives the braid factorization for $V_{2}\left(D_{k}\right)$; the braid factorization of the branch curve $C_{2}$ of $V_{2}$ is computed in $\S 3.3$; the local perturbation procedure to be performed near the intersections of $C_{2}$ with $V_{2}\left(D_{k}\right)$ is described in $\S 3.4 ; \S 3.5$ deals with the 
assembling procedure that yields the braid factorization for $D_{2 k}$ from the previous ingredients ; finally, the calculation is completed in $\S 3.6$.

Remark. More generally, this procedure applies to any situation involving iterated branched coverings : given two approximately holomorphic branched covering maps $f$ and $g$, the composed map $h=g \circ f$ has a non-generic behavior at each of the intersection points of the branch curves of $f$ and $g$; however, the perturbation procedure described in $\S 3.4$ also applies to this situation, and calculations similar to those of Section 3 can be used to compute the braid monodromy of a "generic" perturbation $\tilde{h}$ of $h$.

Also observe that, in the case of complex surfaces, the manner in which we perturb iterated coverings, even though it is not holomorphic, is very similar to the corresponding construction in complex geometry. In particular, even though our computations are always performed up to $m$-equivalence (allowing cancellations of pairs of nodes), in the case of complex manifolds a formula very similar to (1) holds up to Hurwitz and conjugation equivalence (without node cancellations). The only issue requiring particular attention is the manner in which the multiple copies of the curve $C_{2}$ are perturbed away from each other (see §3.6), where the most natural choice in the approximately holomorphic context may be slightly different from a holomorphic perturbation; still, evidence suggests that in practice Theorem 2 does hold up to Hurwitz and conjugation equivalence for most complex surfaces (see the end of $\S 3.6$ ).

Remark. The branched coverings constructed in [5] and the symplectic Lefschetz pencils constructed by Donaldson enjoy transversality properties which intuitively ought to make their topology very special among all possible coverings or pencils. It is therefore interesting to ask for criteria indicating whether a given covering map (or Leschetz pencil) is "of Donaldson type"; more precisely, the question is to decide whether, after stabilizing by repeatedly applying the degree doubling formula, the monodromy data of the given covering map $X \rightarrow \mathbb{C P}^{2}$ eventually coincides with the invariants of $X$ given by Theorem 1 . This question can be reformulated in two equivalent ways (similar statements about Lefschetz pencils can also be considered) : 
1. Given two sets of monodromy invariants representing branched coverings of $\mathbb{C P}^{2}$ with the same total space up to symplectomorphism, do they always become $m$-equivalent to each other by repeatedly applying the degree doubling formula?

2. Is the set of all compact symplectic 4-manifolds with integral symplectic class up to scaling of the symplectic form in bijection with the set of all possible braid factorizations and geometric monodromy representations up to $m$-equivalence and stabilization by degree doubling ?

1.3. Degree doubling for symplectic Lefschetz pencils. A direct application of the degree doubling formula for braid monodromies is a similar formula for the monodromy of the symplectic Lefschetz pencils constructed by Donaldson [10]. Indeed, recall from [10] that every compact symplectic 4-manifold admits a structure of Lefschetz pencil determined by two sections of $L^{\otimes k}$ for large enough $k$. The monodromy of such a Lefschetz pencil is described by a word in the mapping class group of a Riemann surface. As explained in [5], Lefschetz pencils and branched coverings are very closely related to each other, and the monodromy of the Lefschetz pencil can be computed explicitly from the braid factorization and the geometric monodromy representation describing the covering.

More precisely, the geometric monodromy representation $\theta$ determines a group homomorphism $\theta_{*}$ from a subgroup $B_{d}^{0}(\theta)$ of $B_{d}$ to the mapping class group $M_{g}$ of a Riemann surface of genus $g=1-n+(d / 2)$; the braid monodromy is contained in $B_{d}^{0}(\theta)$, and the monodromy of the Lefschetz pencil is obtained by composing the braid monodromy with $\theta_{*}$. It was shown in $\S 5$ of [5] that the nodes and cusps of the branch curve do not contribute to the monodromy of the Lefschetz pencil (the corresponding braids lie in the kernel of $\theta_{*}$ ), while the half-twists corresponding to the tangency points of the branch curve yield Dehn twists in $M_{g}$.

Using this description, we derive in Section 4 a degree doubling formula for Lefschetz pencils. The relation between braid groups and mapping class groups of Riemann surfaces with boundary components is described in more detail in $\S 4.1$, and the degree doubling formula is obtained in $\S 4.2$.

Acknowledgements. We are very grateful to S. Donaldson and M. Gromov for their constant attention to this work. The second author would also like to thank IHES for the extremely pleasant working conditions during part of the 
preparation of this work. Finally, we are very grateful to the referee for his careful reading of the manuscript.

\section{Stably QUASIHOLOMORPHIC COVERINGS}

2.1. Quasiholomorphic coverings and braided curves. We now describe in more detail the geometric properties of the covering maps and branch curves that we will be considering.

Definition 3. A real 2-dimensional singular submanifold $D \subset \mathbb{C P}^{2}$ is a braided curve if it satisfies the following properties : (1) the only singular points of $D$ are cusps (with positive orientation) and transverse double points (with either orientation) ; (2) the point $(0: 0: 1)$ does not belong to $D ;(3)$ the fibers of the projection $\pi:(x: y: z) \mapsto(x: y)$ are everywhere transverse to $D$, except at a finite set of nondegenerate tangency points where a local model for $D$ in orientation-preserving coordinates is $z_{2}^{2}=z_{1}$; (4) the cusps, nodes and tangency points are all distinct and lie in different fibers of $\pi$.

This notion is a topological analogue of the notion of quasiholomorphic curve as described in [5]. In fact, a singular curve in $\mathbb{C P}^{2}$ can be described by a braid factorization with factors of degree $1, \pm 2$, and 3 if and only if it is braided. As observed in [5], every braided curve is isotopic to a symplectic curve, as follows immediately from applying the transformation $(x: y: z) \mapsto(x: y: \epsilon z)$, with $\epsilon$ sufficiently small. However, the branch curves obtained from asymptotically holomorphic families of branched coverings satisfy much more restrictive geometric assumptions.

More precisely, recall that the notion of quasiholomorphicity only makes sense for a sequence of branch curves obtained for increasing values of the degree $k$, and that the resulting geometric estimates improve when $k$ increases. The geometric properties that follow immediately from the definitions and arguments in [1] and [5] are the following. Recall that $(X, \omega)$ is endowed with a compatible almostcomplex structure $J$ and the corresponding metric $g$, and that we rescale this metric to work with the metric $g_{k}=k g$. 
Definition 4. A sequence of sections $s_{k}$ of complex vector bundles $E_{k}$ over $X$ (endowed with Hermitian metrics and connections) is asymptotically holomorphic if there exist constants $C_{j}$ independent of $k$ such that $\left|\nabla^{j} s_{k}\right|_{g_{k}} \leq C_{j}$ and $\left|\nabla^{j-1} \bar{\partial} s_{k}\right|_{g_{k}} \leq C_{j} k^{-1 / 2}$ for all $j$.

The sections $s_{k}$ are uniformly transverse to 0 if there exists a constant $\gamma>0$ such that, at every point $x \in X$ where $\left|s_{k}(x)\right| \leq \gamma$, the covariant derivative $\nabla s_{k}(x)$ is surjective and has a right inverse of norm less than $\gamma^{-1}$ w.r.t. $g_{k}$ (we then say that $s_{k}$ is $\gamma$-transverse to 0 ).

If the sections $s_{k}$ are asymptotically holomorphic and uniformly transverse to 0 then for large $k$ their zero sets are smooth asymptotically holomorphic symplectic submanifolds.

Definition 5. A sequence of branched covering maps $f_{k}: X \rightarrow \mathbb{C P}^{2}$ determined by asymptotically holomorphic sections $s_{k}=\left(s_{k}^{0}, s_{k}^{1}, s_{k}^{2}\right)$ of $\mathbb{C}^{3} \otimes L^{\otimes k}$ for $k \gg 0$ is quasiholomorphic if there exist constants $C_{j}, \gamma, \delta$ independent of $k$, almostcomplex structures $\tilde{J}_{k}$ on $X$, and finite subsets $\mathcal{F}_{k} \subset X$, such that the following properties hold (using $\tilde{J}_{k}$ to define the $\bar{\partial}$ operator) :

(0) $\left|\nabla^{j}\left(\tilde{J}_{k}-J\right)\right|_{g_{k}} \leq C_{j} k^{-1 / 2}$ for every $j \geq 0 ; \tilde{J}_{k}=J$ outside of the $2 \delta$ neighborhood of $\mathcal{F}_{k} ; \tilde{J}_{k}$ is integrable over the $\delta$-neighborhood of $\mathcal{F}_{k}$;

(1) the norm of $s_{k}$ is everywhere bounded from below by $\gamma$; as a consequence, $\left|\nabla^{j} f_{k}\right|_{g_{k}} \leq C_{j}$ and $\left|\nabla^{j-1} \bar{\partial} f_{k}\right|_{g_{k}} \leq C_{j} k^{-1 / 2}$ for all $j ;$

(2) $\left|\nabla f_{k}(x)\right|_{g_{k}} \geq \gamma$ at every point $x \in X$;

(3) the (2,0)-Jacobian $\operatorname{Jac}\left(f_{k}\right)=\operatorname{det} \partial f_{k}$ is $\gamma$-transverse to 0 ; in particular it vanishes transversely along a smooth symplectic curve $R_{k} \subset X$ (the ramification curve).

$\left(3^{\prime}\right)$ the restriction of $\bar{\partial} f_{k}$ to Ker $\partial f_{k}$ vanishes at every point of $R_{k}$;

(4) the quantity $\partial\left(f_{k \mid R_{k}}\right)$, which can be seen as a section of a line bundle over $R_{k}$, is $\gamma$-transverse to 0 and vanishes at a finite subset $\mathcal{C}_{k} \subset \mathcal{F}_{k}$ (the cusp points of $f_{k}$ ) ; in particular $f_{k}\left(R_{k}\right)=D_{k}$ is an immersed symplectic curve away from the image of $\mathcal{C}_{k}$;

(5) $f_{k}$ is $\tilde{J}_{k}$-holomorphic over the $\delta$-neighborhood of $\mathcal{F}_{k}$; 
(6) the section $\left(s_{k}^{0}, s_{k}^{1}\right)$ of $\mathbb{C}^{2} \otimes L^{\otimes k}$ is $\gamma$-transverse to 0 ; as a consequence $D_{k}$ remains away from the point $(0: 0: 1)$;

(7) letting $\phi_{k}=\pi \circ f_{k}: R_{k} \rightarrow \mathbb{C P}^{1}$, the quantity $\partial\left(\phi_{k \mid R_{k}}\right)$ is $\gamma$-transverse to 0 over $R_{k}$, and it vanishes over the union of $\mathcal{C}_{k}$ with a finite set $\mathcal{T}_{k}$ (the tangency points of $D_{k}$ ) ; moreover, $\bar{\partial} f_{k}=0$ at every point of $\mathcal{T}_{k}$;

(8) the projection $f_{k}: R_{k} \rightarrow D_{k}$ is injective outside the singular points of $D_{k}$, and the branch curve $D_{k}$ is braided.

The main result of [5] is the existence, for large enough values of $k$, of quasiholomorphic covering maps $X \rightarrow \mathbb{C P}^{2}$ determined by sections of $\mathbb{C}^{3} \otimes L^{\otimes k}$, canonical up to isotopy. The braid monodromy invariants corresponding to these coverings are those mentioned in Theorem 1.

2.2. Stably quasiholomorphic coverings. We wish to construct and study branched covering maps which, in addition to being quasiholomorphic, behave nicely when composed with a quadratic holomorphic map from $\mathbb{C P}^{2}$ to itself. For this purpose, we extend in the following way the notions defined in the previous sections :

Definition 6. We say that the image $D \subset \mathbb{C P}^{2}$ of a smooth curve $R$ by a map $f$ is locally braided if there exists a finite number of open subsets $U_{j} \subset R$, whose union is $R$, such that for all $j$ the image $f\left(U_{j}\right) \subset D$ is a braided curve in $\mathbb{C P}^{2}$.

In other words, a locally braided curve is similar to a braided curve except that it is merely immersed outside its cusps, without any self-transversality property ; although the cusps and tangencies of a locally braided curve are still nondegenerate and well-defined, phenomena such as self-tangencies might occur. For example, if the definition of a quasiholomorphic covering is relaxed by removing condition (8), the branch curve $D_{k}$ is only locally braided.

Although a locally braided branch curve does not have a well-defined braid monodromy, an arbitrarily small perturbation ensures self-transversality and yields a braided curve ; it is easy to check that the braid monodromies of all possible resulting curves are m-equivalent, as the only phenomenon which can occur in a generic 1-parameter family is the cancellation of pairs of double points.

Definition 7. A sequence of branched covering maps $f_{k}: X \rightarrow \mathbb{C P}^{2}$ determined by asymptotically holomorphic sections $s_{k}=\left(s_{k}^{0}, s_{k}^{1}, s_{k}^{2}\right)$ of $\mathbb{C}^{3} \otimes L^{\otimes k}$ for $k \gg 0$ 
is stably quasiholomorphic if, with the same notations as in Definition 5, the following properties hold:

(1) the covering maps $f_{k}$ are quasiholomorphic;

(2) the sections $s_{k}^{0}, s_{k}^{1}$ and $s_{k}^{2}$ of $L^{\otimes k}$ are $\gamma$-transverse to 0 ;

(3) the sections $\left(s_{k}^{0}, s_{k}^{1}\right),\left(s_{k}^{0}, s_{k}^{2}\right)$ and $\left(s_{k}^{1}, s_{k}^{2}\right)$ of $\mathbb{C}^{2} \otimes L^{\otimes k}$ are $\gamma$-transverse to 0 ;

(4) let $\pi^{0}, \pi^{1}$ and $\pi^{2}$ be the projections $(x: y: z) \mapsto(y: z),(x: y: z) \mapsto(x: z)$ and $(x: y: z) \mapsto(x: y)$ respectively, and define $\phi_{k}^{i}=\pi^{i} \circ f_{k}$; the quantity $\partial\left(\left(\phi_{k}^{i}\right)_{\mid\left(s_{k}^{i}\right)^{-1}(0)}\right)$ is $\gamma$-transverse to 0 over $\left(s_{k}^{i}\right)^{-1}(0)$ for $i=0,1,2$;

(5) the quantity $\left|\partial \phi_{k}^{i}\right|_{g_{k}}$ is bounded from below by $\gamma$ over $\left(s_{k}^{i}\right)^{-1}(0)$;

(6) $\mathcal{F}_{k}=\mathcal{C}_{k} \cup \mathcal{T}_{k} \cup \mathcal{I}_{k}$, where $\mathcal{T}_{k}$ is the set of tangency points and $\mathcal{I}_{k}$ is the set of points of $R_{k}$ where one of the three sections $s_{k}^{i}$ vanishes.

We have the following extension of the main results of [1] and [5], which will be proved in $\S 2.3$ :

Proposition 1. For all large values of $k$, there exist asymptotically holomorphic sections $s_{k}$ of $\mathbb{C}^{3} \otimes L^{\otimes k}$ such that the corresponding projective maps $f_{k}: X \rightarrow \mathbb{C P}^{2}$ are stably quasiholomorphic coverings. Moreover, for large $k$ the topology of these covering maps is canonical up to isotopy and cancellations of pairs of nodes in the branch curve.

More precisely, the uniqueness statement means that, given two sequences of stably quasiholomorphic coverings, it is possible for large $k$ to find an interpolating 1-parameter family of covering maps, all of which are stably quasiholomorphic, except for finitely many parameter values where a cancellation or creation of a pair of nodes occurs in the branch curve.

The following result will be used in $\S 3.2$ to compute the braid monodromy of the folded branch curve $V_{2}^{\prime}\left(D_{k}\right)$ :

Lemma 1. Consider the two maps $V_{2}^{0}:(x: y: z) \mapsto\left(x^{2}: y^{2}: z^{2}\right)$ and $\psi_{a}:(x: y: z) \mapsto(x: a y+(1-a) x: a z+(1-a) x)$ from $\mathbb{C P}^{2}$ to itself, and let $f_{k}$ be a sequence of stably quasiholomorphic covering maps with branch curves $D_{k} \subset$ $\mathbb{C P}^{2}$. Then the curves $V_{2}^{0}\left(D_{k}\right)$ are locally braided. Moreover, if we assume that $(0: 1: 1) \notin D_{k}$ and that none of the nodes of $D_{k}$ lies on the line $L_{0}=\{(0: y: z)\}$, 
then for all sufficiently small non-zero values of $a \in \mathbb{C}$ the curves $V_{2}^{0}\left(\psi_{a}\left(D_{k}\right)\right)$ are locally braided and isotopic to $V_{2}^{0}\left(D_{k}\right)$ through locally braided curves.

Furthermore, these properties remain true if $V_{2}^{0}$ is replaced by any generic holomorphic quadratic map $V_{2}^{\prime}$ from $\mathbb{C P}^{2}$ to itself which differs from $V_{2}^{0}$ by less than $\gamma^{\prime}$ (in $C^{1}$ norm), for some constant $\gamma^{\prime}$ independent of $k$.

(Since sections of $O(2)$ form a finite-dimensional vector space, in the last statement one can use any norm to estimate the difference between $V_{2}^{\prime}$ and $V_{2}^{0}$; but as will be apparent from the proof, it is the $C^{1}$-estimate that is geometrically relevant.)

Proof. The ramification curve of $V_{2}^{0}$ consists of three lines $L_{0}=\{(0: y: z)\}$, $L_{1}=\{(x: 0: z)\}$ and $L_{2}=\{(x: y: 0)\}$. Moreover, $V_{2}^{0}$ maps each fiber of $\pi$ to a fiber of $\pi$. Therefore, let $C \subset \mathbb{C P}^{2}$ be a locally braided curve satisfying the following properties: (a) $C$ is transverse to the lines $L_{0}, L_{1}, L_{2}$ and avoids their intersection points; $(b)$ the cusps and tangency points of $C$ lie away from $L_{0}, L_{1}, L_{2} ;(c)$ at any point $p \in C \cap L_{i}$, the curve $C$ is transverse to the fiber of the projection $\pi^{i}$ through $p ;(d)$ the curve $C$ is holomorphic near its tangency points and near its intersections with $L_{0} \cup L_{1}$. Then we conclude that $V_{2}^{0}(C)$ is locally braided and holomorphic near its tangency points.

Indeed, conditions $(a)-(c)$ imply that the restriction of $V_{2}^{0}$ to $C$ is an immersion, because $V_{2}^{0}$ is a local diffeomorphism away from $L_{i}$, and $C$ is transverse to the kernel of the differential of $V_{2}^{0}$ at its intersection points with $L_{i}$. Moreover, (a) also implies that $V_{2}^{0}(C)$ avoids the point $(0: 0: 1)$. The cusps of $V_{2}^{0}(C)$ are exactly the images of those of $C$, while the tangency points of $V_{2}^{0}(C)$ are of two types: on one hand, the images of the tangency points of $C$, and on the other hand, the images of the intersection points of $C$ with either $L_{0}$ or $L_{1}$. Property $(d)$ implies that $V_{2}^{0}(C)$ is holomorphic near its tangency points, and because $C$ is locally braided and transverse to $L_{0} \cup L_{1}$, these tangencies are non-degenerate, which implies that $V_{2}^{0}(C)$ is locally braided.

We now check that, as a consequence of Definition 7 , the curves $D_{k}$ satisfy properties $(a)-(d)$. Indeed, property (3) of Definition 7 implies that $f_{k}$ is a local diffeomorphism wherever two of the components of $s_{k}$ are very small; therefore $D_{k}$ avoids the intersection points of $L_{0}, L_{1}, L_{2}$. Moreover, property (2) of Definition 7 implies that $\left(s_{k}^{i}\right)^{-1}(0)$ is smooth and $D_{k}$ is transverse to $L_{i}$ for $i=0,1,2$; 
so condition $(a)$ is satisfied. This transversality requirement also implies that the tangency points of $D_{k}$ do not lie on $L_{0}$ or $L_{1}$; in the case of $L_{2}$ we appeal to property (5) of Definition 7 to reach the same conclusion (recall that by definition $\partial \phi_{k}^{2}$ vanishes at tangency points). Furthermore, property (4) of Definition 7 means that the restriction of $\phi_{k}^{i}$ to $\left(s_{k}^{i}\right)^{-1}(0)$ has non-degenerate critical points, which implies that the intersection multiplicity of $R_{k}$ with $\left(s_{k}^{i}\right)^{-1}(0)$ at such a point is always 1 and prevents a cusp of $D_{k}$ from lying on $L_{i}$. Therefore $(b)$ holds.

Condition $(c)$ is a direct consequence of property (5) of Definition 7, observing that the points where $D_{k}$ is tangent to the fiber of $\pi^{i}$ are precisely the critical points of $\phi_{k}^{i}$. Finally, condition $(d)$ follows immediately from property (6) of Definition 7. Therefore $D_{k}$ satisfies $(a)-(d)$, which implies that $V_{2}^{0}\left(D_{k}\right)$ is locally braided and holomorphic near its tangency points.

We now consider the curve $V_{2}^{0}\left(\psi_{a}\left(D_{k}\right)\right)$. Observe that, when $a \rightarrow 0$, the linear map $\psi_{a}$ fixes the points of $L_{0}$ and collapses all other points towards $p_{0}=(1$ : $1: 1)$. Moreover, $\psi_{a}$ maps each fiber of $\pi$ to a fiber of $\pi$. If we assume that the nodes of $D_{k}$ don't lie on $L_{0}$, then for sufficiently small values of $a$ the curve $\psi_{a}\left(D_{k}\right)$ becomes arbitrarily close to a union of $d=\operatorname{deg} D_{k}$ lines, each joining a point of $D_{k} \cap L_{0}$ to $p_{0}$. The requirement $(0: 1: 1) \notin D_{k}$ ensures that none of these lines is a fiber of the projection $\pi^{0}$. The cusps and tangency points of $\psi_{a}\left(D_{k}\right)$ are the images of those of $D_{k}$ and hence all lie in a small ball centered at $p_{0}$; moreover the holomorphicity of $D_{k}$ near the points of $D_{k} \cap L_{0}$ implies that $\psi_{a}\left(D_{k}\right)$ is holomorphic outside of a small ball centered at $p_{0}$. Therefore $\psi_{a}\left(D_{k}\right)$ satisfies the conditions $(a)-(d)$ listed above, and $V_{2}^{0}\left(\psi_{a}\left(D_{k}\right)\right)$ is locally braided and holomorphic near its tangency points for all sufficiently small values of $a$.

Observing that properties $(a)-(c)$ are open conditions, one easily checks that, if the behavior of the curve $D_{k}$ is generic (which can be ensured by a small perturbation), then the curves $\psi_{a}\left(D_{k}\right)$ (or small perturbations thereof) satisfy $(a)-(c)$ for all but a discrete set of values of $a$. Therefore, observing that $\psi_{1}=\mathrm{Id}$ and choosing a suitable path $a(t)$, there exists an isotopy between $D_{k}$ and $\psi_{a}\left(D_{k}\right)$ through braided curves satisfying conditions $(a)-(c)$. Although the possible lack of holomorphicity of $\psi_{a(t)}\left(D_{k}\right)$ near its intersections with $L_{1}$ may prevent $(d)$ from holding, this specific requirement is actually not needed to ensure that $V_{2}^{0}\left(\psi_{a(t)}\left(D_{k}\right)\right)$ is locally braided. Therefore, $V_{2}^{0}\left(\psi_{a}\left(D_{k}\right)\right)$ is isotopic to $V_{2}^{0}\left(D_{k}\right)$ through locally braided curves. 
Finally, we consider a holomorphic quadratic map $V_{2}^{\prime}$ sufficiently close to $V_{2}^{0}$. Our main observation is that the curves $V_{2}^{\prime}\left(D_{k}\right)$ and $V_{2}^{0}\left(D_{k}\right)$ are $C^{1}$-close to each other. Therefore, because $V_{2}^{0}\left(D_{k}\right)$ is locally braided and holomorphic near its tangency points (which are all non-degenerate), the curve $V_{2}^{\prime}\left(D_{k}\right)$ is also locally braided; indeed, if $V_{2}^{\prime}$ is sufficiently close to $V_{2}^{0}$ then every point where $V_{2}^{\prime}\left(D_{k}\right)$ fails to be transverse to the fibers of $\pi$ necessarily lies close to a tangency point of $V_{2}^{0}\left(D_{k}\right)$. Furthermore, choosing a continuous deformation of $V_{2}^{0}$ into $V_{2}^{\prime}$, it is clear that $V_{2}^{0}\left(D_{k}\right)$ and $V_{2}^{\prime}\left(D_{k}\right)$ are isotopic to each other among locally braided curves.

The reason why we can obtain a uniform estimate $\gamma^{\prime}$ on the maximum admissible value of $\left\|V_{2}^{\prime}-V_{2}^{0}\right\|_{C^{1}}$ is the existence of uniform estimates on the geometry of $D_{k}$. Indeed, by carefully keeping track of the uniform estimates given by Definitions 5 and 7 , it is possible to derive uniform lower bounds for all geometrically relevant quantities, such as the distance from $D_{k}$ to the intersection points of the lines $L_{i}$, the transversality angle at the intersections of $D_{k}$ with $L_{i}$, the distance between $L_{i}$ and the cusps and tangency points of $D_{k}$, the second derivative of $\pi_{\mid D_{k}}$ at the tangency points of $D_{k}$ and its first derivative away from these points, ... This yields uniform estimates on the geometry of $V_{2}^{0}\left(D_{k}\right)$ near its tangency points and implies that the property of being locally braided remains valid up to a certain size of perturbation of $V_{2}^{0}$ which can be estimated explicitly in terms of the various bounds.

Moreover, recalling from above the behavior of $\psi_{a}$ for small values of $a$, we can similarly show that if $a$ is sufficiently small then $V_{2}^{\prime}\left(\psi_{a}\left(D_{k}\right)\right)$ is locally braided and isotopic to $V_{2}^{0}\left(\psi_{a}\left(D_{k}\right)\right)$ through locally braided curves; one simply needs to choose $V_{2}^{\prime}$ generic in order to ensure that the images by $V_{2}^{\prime}$ of the lines joining $p_{0}$ to the points of $D_{k} \cap L_{0}$ are smooth conics.

We conclude in particular that the images by $V_{2}^{0}$ and $V_{2}^{\prime}$ of $D_{k}$ and $\psi_{a}\left(D_{k}\right)$ are all mutually isotopic among locally braided curves, and their braid monodromies are m-equivalent to each other.

The following observation plays a crucial role in our strategy to prove Theorem 2: given a generic holomorphic quadratic map $V_{2}^{\prime}$ close to $V_{2}^{0}$, the composed maps $V_{2}^{\prime} \circ f_{k}$ already satisfy most of the properties expected of quasiholomorphic coverings except at the points where the branch curve of $f_{k}$ intersects that of $V_{2}^{\prime}$. 
Proposition 2. Let $f_{k}$ be a family of stably quasiholomorphic coverings, and let $V_{2}^{\prime}$ be a generic holomorphic quadratic map close to $V_{2}^{0}$. Then, given any fixed constant $d_{0}>0$, there exist constants $C_{j}, \gamma, \delta$ independent of $k$ (but depending on $V_{2}^{\prime}$ and on $d_{0}$ ) such that the composed maps $f_{2 k}^{\prime}=V_{2}^{\prime} \circ f_{k}$ satisfy all the properties of Definition 5, except for properties ( $\left.3^{\prime}\right)$ and (8), at every point of $X$ whose $g_{k}$-distance to $\mathcal{I}_{k}^{\prime}=R_{k} \cap f_{k}^{-1}\left(R_{2}^{\prime}\right)$ is larger than $d_{0}\left(R_{k}\right.$ and $R_{2}^{\prime}$ are the ramification curves of $f_{k}$ and $V_{2}^{\prime}$ respectively).

Proof. The projective map $f_{2 k}^{\prime}=V_{2}^{\prime} \circ f_{k}$ is defined by a section $Q\left(s_{k}\right)$ of $\mathbb{C}^{3} \otimes L^{\otimes 2 k}$, each of its three components being a quadratic expression $Q_{i}\left(s_{k}\right)(0 \leq i \leq 2)$ in the three sections defining $f_{k}$. It is therefore easy to show that the sections $Q\left(s_{k}\right)$ are asymptotically holomorphic.

Because the projective map $V_{2}^{\prime}$ induced by the polynomials $Q_{i}$ is well-defined, the inequality $|Q(s)| \geq c|s|^{2}$ holds for some constant $c>0$. Therefore, the existence of a uniform lower bound on $\left|s_{k}\right|$ at every point of $X$ implies that of a uniform lower bound on $\left|Q\left(s_{k}\right)\right|$, and so property (1) of Definition 5 is satisfied everywhere.

As observed above, by property (2) of Definition 7 the branch curve of $f_{k}$ is uniformly transverse to the ramification curve of $V_{2}^{0}$ and hence to that of $V_{2}^{\prime}$. Therefore, if a point $x \in X$ lies close both to $R_{k}$ and to $f_{k}^{-1}\left(R_{2}^{\prime}\right)$ then it always lies close to a point of $\mathcal{I}_{k}^{\prime}$.

Property (2) of quasiholomorphic coverings follows from the observation that, since the differentials of $f_{k}$ and $V_{2}^{\prime}$ both have complex rank at least 1 everywhere, $\nabla f_{2 k}^{\prime}(x)$ can only be small if the Jacobians of $f_{k}$ at $x$ and of $V_{2}^{\prime}$ at $f_{k}(x)$ are both small. These quantities vanish transversely $\left(f_{k}\right.$ is quasiholomorphic and $V_{2}^{\prime}$ is generic), so $x$ must lie close to both branch curves, and hence, by the above observation, close to $\mathcal{I}^{\prime}{ }_{k}$ (closer than $d_{0}$ if $\left|\nabla f_{2 k}^{\prime}(x)\right|$ is assumed small enough). In fact, $\left|\nabla f_{2 k}^{\prime}\right|$ remains bounded away from 0 even near $\mathcal{I}_{k}^{\prime}$, because, as observed in the proof of Lemma 1, property (5) of Definition 7 implies that $V_{2}^{0}$ (and hence also $\left.V_{2}^{\prime}\right)$ restricts to the branch curve of $f_{k}$ as an immersion.

We now turn to the third property. The $(2,0)$-Jacobian of $f_{2 k}^{\prime}$ is given by $\operatorname{Jac}\left(f_{2 k}^{\prime}\right)=\operatorname{Jac}\left(f_{k}\right) \cdot f_{k}^{*} \operatorname{Jac}\left(V_{2}^{\prime}\right)$. It can only be small when one of the two terms in the product is small, i.e. near one of the two branch curves. Moreover, $f_{k}^{*} \operatorname{Jac}\left(V_{2}^{\prime}\right)$ is bounded away from zero everywhere except near $f_{k}^{-1}\left(R_{2}^{\prime}\right)$, so the transverse 
vanishing of $\operatorname{Jac}\left(f_{k}\right)$ implies that of $\operatorname{Jac}\left(f_{2 k}^{\prime}\right)$ at these points. Similarly $\operatorname{Jac}\left(f_{k}\right)$ is bounded from below everywhere except near $R_{k}$, so the transverse vanishing of $f_{k}^{*} \mathrm{Jac}\left(V_{2}^{\prime}\right)$ implies the desired property at these points. As a consequence the transversality to 0 of $\operatorname{Jac}\left(f_{2 k}^{\prime}\right)$ holds everywhere except near $\mathcal{I}_{k}^{\prime}$ (note that the obtained transversality estimate has to be decreased when $d_{0}$ becomes smaller).

We now look at property (4). Away from $\mathcal{I}_{k}^{\prime}$ the branch curve of $f_{2 k}^{\prime}$ consists of two separate components, $R_{k}$ and $f_{k}^{-1}\left(R_{2}^{\prime}\right)$, so we work separately on each component. We first look at $R_{k}$ : we know that $\partial\left(f_{k \mid R_{k}}\right)$ is uniformly transverse to 0 , and given a point $p \in R_{k}$ at distance more than $d_{0}$ from $\mathcal{I}_{k}^{\prime}$, the complex linear map $\nabla V_{2}^{\prime}$ is an isomorphism at $f_{k}(p)$, with norm bounded from below (the constant depends on $\left.d_{0}\right)$. Composing $\partial\left(f_{k \mid R_{k}}\right)$ with $\nabla V_{2}^{\prime}$, we obtain that $\partial\left(f_{2 k \mid R_{k}}^{\prime}\right)$ is also uniformly transverse to 0 at all points of $R_{k}$ at distance more than $d_{0}$ from $\mathcal{I}_{k}^{\prime}$ (again, the constant depends on $d_{0}$ ). The argument works similarly on $f_{k}^{-1}\left(R_{2}^{\prime}\right)$ : away from $\mathcal{I}_{k}^{\prime}, \partial f_{k}$ is an isomorphism with norm bounded from below (the constant depends on $d_{0}$ ), and because $V_{2}^{\prime}$ has been chosen generic the quantity $\nabla\left(V_{2 \mid R_{2}^{\prime}}^{\prime}\right)$ vanishes transversely, so $\partial\left(f_{2 k \mid f_{k}^{-1}\left(R_{2}^{\prime}\right)}^{\prime}\right)$ is uniformly transverse to 0 at all points of $f_{k}^{-1}\left(R_{2}^{\prime}\right)$ at distance more than $d_{0}$ from $\mathcal{I}_{k}^{\prime}$.

Observe by the way that all cusp points of $f_{k}$ and of $V_{2}^{\prime}$ lie away from $\mathcal{I}_{k}^{\prime}$. Indeed, for the cusp points of $f_{k}$ it follows from property (4) in Definition 7 that they lie away from the branch curve of $V_{2}^{0}$ and hence from that of $V_{2}^{\prime}$, as observed in the proof of Lemma 1 . On the other hand, is easy to see that the cusp points of $V_{2}^{\prime}$ all lie close to one of the three singular points of $V_{2}^{0}$, while property (3) in Definition 7 implies that the branch curve of $f_{k}$ remains far away from these points.

Property (5) is easy to check: since compatible almost-complex structures on $X$ are sections of a bundle with contractible fiber, it is sufficient to work locally near a cusp point. The points we have to consider are either cusp points of $f_{k}$ or the preimages by $f_{k}$ of those of $V_{2}^{\prime}$. In the first case, it is sufficient to choose the same almost-complex structure $\tilde{J}_{k}$ as for $f_{k}$, because $V_{2}^{\prime}$ is holomorphic. In the second case, consider the pull-back $f_{k}^{*} \mathbb{J}_{0}$ of the standard complex structure of $\mathbb{C P}^{2}$ via the map $f_{k}$. Since all cusp points of $V_{2}^{\prime}$ lie far from the branch curve of $f_{k}$, the differential of $f_{k}$ is locally an isomorphism and satisfies a uniform lower bound. Therefore the asymptotic holomorphicity of the sections defining $s_{k}$ is enough to ensure that $f_{k}^{*} \mathbb{J}_{0}$ differs from $J$ by at most $O\left(k^{-1 / 2}\right)$ in any $C^{r}$ norm. 
A standard argument involving a smooth cut-off function can be used in order to define a smooth almost-complex structure which coincides with $f_{k}^{*} \mathbb{J}_{0}$ near the cusp point and with $J$ outside a small ball.

We now turn to property (6). Consider a point $x \in X$ where the first two sections defining $f_{2 k}^{\prime}$, namely $Q_{0}\left(s_{k}\right)$ and $Q_{1}\left(s_{k}\right)$, are both very small. Because the quadratic map $V_{2}^{\prime}$ is close to $V_{2}^{0}$, and because the only preimage of $(0: 0: 1)$ by $V_{2}^{0}$ is $(0: 0: 1)$ itself, the quantities $s_{k}^{0}(x)$ and $s_{k}^{1}(x)$ are also small. So, if we assume that $\left|V_{2}^{\prime}-V_{2}^{0}\right|_{C^{1}}$ is sufficiently small, the uniform transversality property of $\left(s_{k}^{0}, s_{k}^{1}\right)$ provides a lower bound on $\operatorname{Jac}\left(f_{k}\right)(x)$. On the other hand, if $V_{2}^{\prime}$ is chosen generic, then its branch curve avoids the point $(1: 0: 0)$ by a certain distance $\rho>0$. Therefore, if $Q_{0}\left(s_{k}\right)$ and $Q_{1}\left(s_{k}\right)$ are sufficiently small, then $f_{2 k}^{\prime}(x)$ lies at distance at least $\rho / 2$ from the branch curve of $V_{2}^{\prime}$. and we can obtain a uniform lower bound (depending on $\rho$ only) on the Jacobian of $V_{2}^{\prime}$ at $f_{k}(x)$. It follows that $\operatorname{Jac}\left(f_{2 k}^{\prime}\right)(x)=\operatorname{Jac}\left(f_{k}\right)(x) \operatorname{Jac}\left(V_{2}^{\prime}\right)\left(f_{k}(x)\right)$ is bounded from below by a fixed constant independently of $k$. Because of the $C^{1}$ bounds on $Q_{i}\left(s_{k}\right)$, we conclude that the covariant derivative of $\left(Q_{0}\left(s_{k}\right), Q_{1}\left(s_{k}\right)\right)$ at $x$ is surjective and bounded from below by a uniform constant. So property (6) holds.

We finally look at property (7), which actually is equivalent to the requirement that the branch curve be locally braided. Most of the work has already been done in the proof of Lemma 1. More precisely, after removing the intersection $\mathcal{I}_{k}^{\prime}$, the branch curve of $f_{2 k}^{\prime}$ splits into the two components $R_{k}$ and $f_{k}^{-1}\left(R_{2}^{\prime}\right)$, and we consider them separately. The critical points of $\psi_{k}^{0}=\left(\pi \circ V_{2}^{0} \circ f_{k}\right)_{\mid R_{k}}$ and $\psi_{k}^{\prime}=\left(\pi \circ f_{2 k}^{\prime}\right)_{\mid R_{k}}$ correspond to the cusps and tangency points of $V_{2}^{0}\left(D_{k}\right)$ and $V_{2}^{\prime}\left(D_{k}\right)$, respectively. Therefore, we have seen in the proof of Lemma 1 that all the critical points of $\psi_{k}^{0}$, and hence those of $\psi_{k}^{\prime}$, are non-degenerate, with a uniform estimate; moreover, they all lie in a neighborhood of $\mathcal{C}_{k} \cup \mathcal{T}_{k} \cup \mathcal{I}_{k}$, which implies that $f_{k}$ is locally holomorphic with respect to a suitable almost-complex structure.

We now look at the component $f_{k}^{-1}\left(R_{2}^{\prime}\right)$ away from the points of $\mathcal{I}_{k}^{\prime}$ : since $f_{k}$ is a local diffeomorphism at all such points, the expected uniform transversality of $\partial\left(\pi \circ f_{2 k}^{\prime}\right)$ is equivalent to the same property for $\partial\left(\pi \circ V_{2}^{\prime}\right)$ restricted to $R_{2}^{\prime}$. However it is easy to check that such a transversality property holds as soon as $V_{2}^{\prime}$ is chosen generic (actually, as soon as $V_{2}^{\prime}\left(R_{2}^{\prime}\right)$ is locally braided). Of course the transversality estimate on $\partial\left(\pi \circ f_{2 k}^{\prime}\right)$ depends on the distance $d_{0}$, because a 
lower bound on $\partial f_{k}$ is used when lifting the transversality property from $\pi \circ V_{2}^{\prime}$ to $\pi \circ f_{2 k}^{\prime}$. Also observe that the holomorphicity of $V_{2}^{\prime}$ implies that the differential of $\pi \circ V_{2 \mid R_{2}^{\prime}}^{\prime}$ vanishes completely at the tangency points of the branch curve of $V_{2}^{\prime}$ (these are genuine tangencies); this clearly implies the same property for $\pi \circ f_{2 k}^{\prime}$ at the tangency points coming from $f_{k}^{-1}\left(R_{2}^{\prime}\right)$. This concludes the proof.

Proposition 2 implies that we can proceed in the following way to construct quasiholomorphic coverings given by sections of $L^{\otimes 2 k}$ for large $k$ : first construct stably quasiholomorphic coverings $f_{k}$ as given by Proposition 1 ; then, define $f_{2 k}^{\prime}=V_{2}^{\prime} \circ f_{k}$ for a generic perturbation $V_{2}^{\prime}$ of $V_{2}^{0}$; and finally perturb $f_{2 k}^{\prime}$ in order to get quasiholomorphic coverings.

Following the arguments in [1] and [5] (see also [2] and the argument in $§ 2.3$ below), we can make the following observations concerning the process by which the maps $f_{2 k}^{\prime}$ are perturbed and made quasiholomorphic. The first step of the construction of quasiholomorphic coverings is to ensure that all the required uniform transversality properties are satisfied over all of $X$. This process is a purely local iterative construction, so that when one starts with $f_{2 k}^{\prime}$ it is sufficient to perturb the given sections of $L^{\otimes 2 k}$ near the points of $\mathcal{I}_{k}^{\prime}$, or equivalently near the points of $\mathcal{I}_{k}$; the required perturbation can be chosen smaller than any fixed given constant (independent of $k$ ), so that it does not significantly affect the topology of $f_{2 k}^{\prime}$ away from the points of $\mathcal{I}_{k}^{\prime}$. The next step in order to construct quasiholomorphic coverings is to ensure property (5) of Definition 5 at the cusp points as well as the last requirement of property (7) at the tangency points ; since the necessary perturbation is bounded by a fixed multiple of $k^{-1 / 2}$, it has no effect whatsoever on braid monodromy outside of a fixed small neighborhood of $\mathcal{I}_{k}^{\prime}$.

At this point in the construction, the branch curves are already locally braided and therefore have well-defined braid monodromies up to $m$-equivalence ; ensuring the remaining conditions $\left(3^{\prime}\right)$ and (8) has no effect on the monodromy data. More precisely, the self-transversality of the branch curves (condition (8)) is obtained by an arbitrarily small perturbation, which is precisely how one defines the braid factorization associated to a locally braided curve. Meanwhile, condition $\left(3^{\prime}\right)$ is obtained by a perturbation process which does not affect the branch curve (see [5]). Finally, notice that, once the covering maps $f_{2 k}^{\prime}$ are perturbed and made 
quasiholomorphic, the braid monodromy invariants associated to them must coincide with those associated to $f_{2 k}$, at least provided that $k$ is large enough : this is a direct consequence of the uniqueness result of [5].

As a consequence of these observations, by computing the braid factorization corresponding to the branch curve of $f_{2 k}^{\prime}$ (very singular, with components of large multiplicity), a great step towards computing the braid factorization for $f_{2 k}$ is already accomplished : the only remaining task is to understand the effect on braid factorizations of the perturbation performed near the points of $\mathcal{I}_{k}^{\prime}$. This justifies the strategy of proof used in $\S 3$.

2.3. Proof of Proposition 1. Proposition 1 can be proved using the same techniques as in [1] and [5] (see also [2]) ; however, the result of [3] can be used to greatly simplify the argument. Observe that the properties expected of $s_{k}$ are of two types : on one hand, uniform transversality properties, which are open conditions on the holomorphic part of the jet of $s_{k}$, and on the other hand, compatibility properties, involving the vanishing of certain antiholomorphic derivatives along the branch curve. The proof therefore consists of two parts. In the first part, successive perturbations of $s_{k}$ are performed in order to achieve the various required transversality properties ; each perturbation is chosen small enough in order to preserve the previously obtained transversality properties. In the second part, $s_{k}$ is perturbed along the curve $R_{k}$ by at most a fixed multiple of $k^{-1 / 2}$ in order to obtain the compatibility conditions.

The first part of the argument can be either carried out as in [1] and [5], or more efficiently by using the result of [3] in the following manner.

Let $E_{k}=\mathbb{C}^{3} \otimes L^{\otimes k}$, and consider the holomorphic jet bundles $\mathcal{J}^{2} E_{k}=E_{k} \oplus$ $T^{*} X^{(1,0)} \otimes E_{k} \oplus\left(T^{*} X^{(1,0)}\right)_{\mathrm{sym}}^{\otimes 2} \otimes E_{k}$. We define the holomorphic 2-jet $j^{2} s$ of a section $s \in \Gamma\left(E_{k}\right)$ as $\left(s, \partial s, \partial(\partial s)_{\mathrm{sym}}\right)$, discarding the antiholomorphic terms or the antisymmetric part of $\partial \partial s$ (these terms are bounded by $O\left(k^{-1 / 2}\right)$ for asymptotically holomorphic sections). Recall from [3] the notion of finite Whitney quasi-stratification of a jet bundle :

Definition 8. Let $(A, \prec)$ be a finite set carrying a binary relation without cycles (i.e., $a_{1} \prec \cdots \prec a_{p} \Rightarrow a_{p} \nprec a_{1}$ ). A finite Whitney quasi-stratification of $\mathcal{J}^{2} E_{k}$ indexed by $A$ is a collection $\left(S^{a}\right)_{a \in A}$ of smooth submanifolds of $\mathcal{J}^{2} E_{k}$, transverse to the fibers, not necessarily mutually disjoint, with the following properties : (1) 
$\partial S^{a}=\overline{S^{a}}-S^{a} \subseteq \bigcup_{b \prec a} S^{b} ;(2)$ given any point $p \in \partial S^{a}$, there exists $b \prec a$ such that $p \in S^{b}$ and such that either $S^{b} \subset \partial S^{a}$ and the Whitney regularity condition is satisfied at all points of $S^{b}$, or $p \notin \Theta_{S^{b}}$, where $\Theta_{S^{b}} \subset S^{b}$ is the set of points where the 2-jet of a section of $E_{k}$ can intersect $S^{b}$ transversely (in particular $\Theta_{S^{b}}=\emptyset$ whenever $\operatorname{codim}_{\mathbb{C}} S^{b}>2$ ).

As in [3], say that a sequence of finite Whitney quasi-stratifications $\mathcal{S}_{k}$ of $\mathcal{J}^{2} E_{k}$ is asymptotically holomorphic if all the strata are approximately holomorphic submanifolds of $\mathcal{J}^{2} E_{k}$, with uniform bounds on the curvature of the strata and on their transversality to the fibers of $\mathcal{J}^{2} E_{k}$.

It was shown in [3] that, given asymptotically holomorphic finite Whitney quasi-stratifications $\mathcal{S}_{k}$ of $\mathcal{J}^{2} E_{k}$, it is always possible for large enough $k$ to construct asymptotically holomorphic sections of $E_{k}$ whose 2-jets are uniformly transverse to the strata of $\mathcal{S}_{k}$; moreover, these sections can be chosen arbitrarily close to any given asymptotically holomorphic sections of $E_{k}$. The result also holds for one-parameter families of sections, which implies that the constructed sections are, for large $k$, canonical up to isotopy.

Using local approximately holomorphic sections of $L^{\otimes k}$ and coordinates over $X$, the fibers of $\mathcal{J}^{2} E_{k}$ can be identified with the space $\mathcal{J}_{2,3}^{2}$ of jets of holomorphic maps from $\mathbb{C}^{2}$ to $\mathbb{C}^{3}$. It was observed in [3] that, if a sequence of finite Whitney quasi-stratifications of $\mathcal{J}^{2} E_{k}$ is such that by this process the restrictions of $\mathcal{S}_{k}$ to the fibers of $\mathcal{J}^{2} E_{k}$ are all identified with a fixed given finite Whitney quasistratification of $\mathcal{J}_{2,3}^{2}$ by complex submanifolds, then the quasi-stratifications $\mathcal{S}_{k}$ are asymptotically holomorphic.

We define finite Whitney quasi-stratifications of $\mathcal{J}^{2} E_{k}$ in the following way. Consider the symmetric holomorphic part $j^{2} s(x)$ of the 2-jet of a section $s=$ $\left(s^{0}, s^{1}, s^{2}\right) \in \Gamma\left(E_{k}\right)$ at a point $x \in X$; if $s(x) \neq 0$, denote by $f$ the corresponding $\mathbb{C P}^{2}$-valued map, and by $\phi^{i}(i \in\{0,1,2\})$ its projections to $\mathbb{C P}^{1}$ along coordinate axes if they are well-defined. Finally, if Jac $f(x)=\wedge^{2} \partial f(x)=0$ and $\partial \mathrm{Jac} f(x)_{\mathrm{sym}}=(\partial \partial f(x))_{\mathrm{sym}} \wedge \partial f(x) \neq 0$, call $R_{x}$ the kernel of the $(1,0)$-form $\partial \mathrm{Jac} f(x)_{\text {sym }}$; one easily checks that $R_{x}$ is well defined in terms of $j^{2} s$ only and that it differs from the tangent space at $x$ to the ramification curve of $f$ by at most $O\left(k^{-1 / 2}\right)$. We define the following submanifolds of $\mathcal{J}^{2} E_{k}$ (in the last two 
definitions, $\{i, j, k\}=\{0,1,2\})$ :

$$
\begin{aligned}
Z & =\left\{j^{2} s(x), s(x)=0\right\} & \text { (codim. 3) } \\
Z_{i j} & =\left\{j^{2} s(x), s^{i}(x)=s^{j}(x)=0\right\} & \text { (codim. 2) } \\
Z_{i} & =\left\{j^{2} s(x), s^{i}(x)=0\right\} & \text { (codim. 1) } \\
\Sigma^{2} & =\left\{j^{2} s(x), s(x) \neq 0, \partial f(x)=0\right\} & \text { (codim. 4) } \\
\Sigma^{1} & =\left\{j^{2} s(x) \notin Z, \partial f(x) \neq 0, \operatorname{Jac} f(x)=0\right\} & \text { (codim. 1) } \\
\Sigma_{s}^{1} & =\left\{j^{2} s(x) \in \Sigma^{1}, \partial \mathrm{Jac} f(x)_{\operatorname{sym}}=0\right\} & \text { (codim. } 3) \\
\Sigma^{1,1} & =\left\{j^{2} s(x) \in \Sigma^{1}-\Sigma_{s}^{1}, \partial f(x)_{\mid R_{x}}=0\right\} & \text { (codim. 2) } \\
\Sigma_{t}^{1} & =\left\{j^{2} s(x) \in \Sigma^{1}-Z_{01}, \partial \phi^{2}(x)=0\right\} & \text { (codim. 2) } \\
\Sigma_{t}^{1,1} & =\Sigma^{1,1} \cap \Sigma_{t}^{1} & \text { (codim. 3) } \\
S_{i} & =\left\{j^{2} s(x) \in Z_{i}-Z_{j k}, \partial \phi^{i}(x)=0\right\} & \text { codim. 3) } \\
S_{i}^{\prime} & =\left\{j^{2} s(x) \in Z_{i}-Z_{j k}, \partial s^{i}(x) \neq 0, \partial \phi^{i}(x)_{\mid \operatorname{Ker} \partial s^{i}(x)}=0\right\} & \text { (codim. 2) }
\end{aligned}
$$

One easily checks that all these subsets are smooth submanifolds of $\mathcal{J}^{2} E_{k}$. Moreover, $Z, Z_{i}$ and $Z_{i j}$ are closed ; $\partial \Sigma^{2} \subseteq Z ; \partial \Sigma^{1}$ and $\partial \Sigma_{s}^{1}$ are contained in $\Sigma^{2} \cup Z ; \partial \Sigma^{1,1} \subseteq \Sigma_{s}^{1} \cup \Sigma^{2} \cup Z ; \partial \Sigma_{t}^{1} \subseteq \Sigma^{2} \cup Z \cup\left(Z_{01}-\Theta_{Z_{01}}\right) ; \partial \Sigma_{t}^{1,1} \subseteq$ $\Sigma_{s}^{1} \cup \Sigma^{2} \cup Z \cup\left(Z_{01}-\Theta_{Z_{01}}\right) ; \partial S_{i} \subseteq\left(Z_{j k}-\Theta_{Z_{j k}}\right) ; \partial S_{i}^{\prime} \subseteq\left(Z_{j k}-\Theta_{Z_{j k}}\right) \cup\left(Z_{i}-\Theta_{Z_{i}}\right)$. Therefore, these submanifolds define quasi-stratifications $\mathcal{S}_{k}$ of $\mathcal{J}^{2} E_{k}$. Note that, because $\Sigma_{s}^{1}=\Sigma^{1}-\Theta_{\Sigma^{1}}$, the stratum $\Sigma_{s}^{1}$ can in fact be eliminated from this description. Moreover, if one uses local approximately holomorphic coordinates and asymptotically holomorphic sections of $L^{\otimes k}$ to trivialize $\mathcal{J}^{2} E_{k}$, it is easy to see that the resulting picture is the same above every point of $X$ : the submanifolds in $\mathcal{S}_{k}$ are identified with holomorphic submanifolds of $\mathcal{J}_{2,3}^{2}$ defined by the same equations. Therefore, by [3] the quasi-stratifications $\mathcal{S}_{k}$ are asymptotically holomorphic.

It is easy to see that conditions (1), (2), (3), (4) and (6) of Definition 5 are equivalent to the uniform transversality of $j^{2} s_{k}$ to $Z, \Sigma^{2}, \Sigma^{1}, \Sigma^{1,1}$ and $Z_{01}$, respectively. Similarly, conditions (2) and (3) of Definition 7 correspond to the uniform transversality of $j^{2} s_{k}$ to $Z_{i}$ and $Z_{i j}$ respectively. Observing that $\partial\left(\phi_{k \mid R_{k}}\right)$ can only vanish at a point $x \in R_{k}$ if either $\partial \phi_{k}(x)=0$ or $\partial\left(f_{k \mid R_{k}}\right)$ vanishes at $x$, we can rephrase condition (7) of Definition 5 in terms of uniform transversality to the singular submanifold of $\mathcal{J}^{2} E_{k}$ consisting of the union of $\Sigma^{1,1}$ (cusp points) 
and $\Sigma_{t}^{1}$ (tangencies), intersecting regularly along $\Sigma_{t}^{1,1}$ ("vertical" cusp points). Therefore, it is equivalent to the uniform transversality of $j^{2} s_{k}$ to $\Sigma^{1,1}, \Sigma_{t}^{1}$, and $\Sigma_{t}^{1,1}$. Finally, conditions (4) and (5) of Definition 7 correspond to the uniform transversality of $j^{2} s_{k}$ to $S_{i}^{\prime}$ and $S_{i}$ respectively.

So, the uniform transversality of $j^{2} s_{k}$ to the quasi-stratifications $\mathcal{S}_{k}$, as given by the main result of [3] provided that $k$ is large enough, is equivalent to the various transversality requirements listed in Definitions 5 and 7 . Moreover, the sections of $\mathbb{C}^{3} \otimes L^{\otimes k}$ constructed in this manner are canonical up to isotopy, as follows from Theorem 3.2 of [3] : given any two sequences of such sections, it is possible for large enough $k$ to find one-parameter families of sections of $\mathbb{C}^{3} \otimes L^{\otimes k}$ interpolating between them and enjoying the same uniform transversality properties for all parameter values.

We now turn to the second part of the argument, namely obtaining the other required properties by perturbing the sections $s_{k}$ by at most $O\left(k^{-1 / 2}\right)$, which clearly affects neither holomorphicity nor transversality properties. The argument is exactly the same as in [5] ; the only difference is that the set $\mathcal{F}_{k}$ of points where the map $f_{k}$ must made holomorphic with respect to a slightly perturbed almostcomplex structure is now slightly larger : one now sets $\mathcal{F}_{k}=\mathcal{C}_{k} \cup \mathcal{I}_{k} \cup \mathcal{I}_{k}$ instead of $\mathcal{F}_{k}=\mathcal{C}_{k}$.

As in [1] and [5], one first chooses suitable almost-complex structures $\tilde{J}_{k}$ differing from $J$ by $O\left(k^{-1 / 2}\right)$ and integrable near the finite set $\mathcal{F}_{k}$. It is then possible to perturb $f_{k}$ near these points in order to obtain condition (5) of Definition 5 , by the same argument as in $\S 4.1$ of [1]. Next, a generic small perturbation yields the self-transversality of $D_{k}$ (property (8) of Definition 5). Finally, a suitable perturbation of $f_{k}$, supported near $R_{k}$ and vanishing near the points of $\mathcal{F}_{k}$, yields property $\left(3^{\prime}\right)$ of Definition 5 along the branch curve, without modifying $R_{k}$ and $D_{k}$, and therefore without affecting the previously obtained compatibility properties. As shown in [5] these various constructions can be performed in oneparameter families, except for property (8) of Definition 5 where cancellations of pairs of nodes must be allowed ; this yields the desired result of uniqueness up to isotopy, and completes the proof of Proposition 1. 


\section{The Degree Doubling Formula FOR BRAID MONODROMIES}

3.1. Generalities about the braid group. We begin by recalling general definitions and notations concerning the braid group on $d$ strings. Consider a set $P=\left\{p_{1}, \ldots, p_{d}\right\}$ of $d$ points in the plane, and recall that $B_{d}=\pi_{0} \operatorname{Diff}_{c}^{+}\left(\mathbb{R}^{2}, P\right)$ is by definition the group of equivalence classes of compactly supported orientationpreserving diffeomorphisms of the plane which leave invariant the set $P$, where two diffeomorphisms are equivalent if and only if they induce the same automorphism of $\pi_{1}\left(\mathbb{R}^{2}-P\right)$. Equivalently $B_{d}$ can be considered as the fundamental group of the configuration space of $d$ points in the plane : a braid corresponds to a motion of the points $p_{1}, \ldots, p_{d}$ such that they remain distinct at all times and eventually return to their original positions (but possibly in a different order) up to homotopy. An important subgroup of $B_{d}$ is the group of pure braids $P_{d}$ (the braids which preserve each of the points $p_{1}, \ldots, p_{d}$ individually) ; it is clear that $B_{d} / P_{d}$ is the symmetric group $S_{d}$.

We will place the points $p_{1}, \ldots, p_{d}$ in that order on the real axis, and denote by $X_{i}$ the positive (counterclockwise) half-twist along the line segment joining $p_{i}$ to $p_{i+1}$, for each $1 \leq i \leq d-1$. It is a classical fact that $B_{d}$ is generated by the $d-1$ half-twists $X_{i}$, and that the relations between them are $X_{i} X_{j}=X_{j} X_{i}$ whenever $|i-j|>1$ and $X_{i} X_{i+1} X_{i}=X_{i+1} X_{i} X_{i+1}$. The center of the braid group is generated by the element $\Delta_{d}^{2}=\left(X_{1} \ldots X_{d-1}\right)^{d}$, which corresponds to rotating everything by $2 \pi$.

We will be especially interested in the half-twists

$$
Z_{i j}=X_{j-1} \cdot \ldots \cdot X_{i+1} \cdot X_{i} \cdot X_{i+1}^{-1} \cdot \ldots \cdot X_{j-1}^{-1} \quad(1 \leq i<j \leq d) .
$$

In this expression of $Z_{i j}$ as a conjugate of $X_{i}$, when $j=i+1$ the conjugating element is a trivial product (ranging over the empty set), and we just have $Z_{i, i+1}=X_{i}$.

The braid $Z_{i j}$ is a positive half-twist along a path joining the points $p_{i}$ and $p_{j}$ and passing above all the points inbetween :

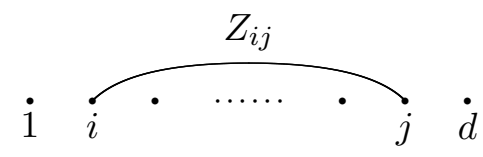


Note that $Z_{i j}$ commutes with $Z_{k l}$ whenever $i<j<k<l$ or $i<k<l<j$. Other useful relations are $Z_{i j} Z_{i k}=Z_{i k} Z_{j k}=Z_{j k} Z_{i j}$ whenever $i<j<k$ (these three expressions differ by a Hurwitz move).

The following factorization of $\Delta^{2}$ as a product of half-twists corresponds to the braid monodromy of a smooth curve of degree $d$ in $\mathbb{C P}^{2}$ (see [12]) :

$$
\Delta_{d}^{2}=\left(X_{1} \ldots X_{d-1}\right)^{d} .
$$

Another important factorization is

$$
\Delta_{d}^{2}=\prod_{i=1}^{d-1} \prod_{j=i+1}^{d} Z_{i j}^{2}=\prod_{i=2}^{d} \prod_{j=1}^{i-1} Z_{j i}^{2}
$$

(these two expressions are clearly Hurwitz equivalent). This factorization corresponds to the braid monodromy of a union of $d$ lines in generic position (see [12]).

We now turn to geometric monodromy representations. Consider the branch curve $D$ of an $n$-sheeted branched covering over $\mathbb{C P}^{2}$, and fix geometric generators $\gamma_{1}, \ldots, \gamma_{d}$ of $\pi_{1}\left(\mathbb{C P}^{2}-D\right)$ (small loops going around the $d=\operatorname{deg} D$ intersection points of $D$ with a given generic fiber of the projection $\pi$ ). It is then possible to define as in $\S 1.1$ the geometric monodromy representation $\theta: F_{d} \rightarrow S_{n}$ associated to the covering. As observed in $\S 1.1$, the fact that the product $\gamma_{1} \cdots \cdots \gamma_{d}$ is trivial in $\pi_{1}\left(\mathbb{C P}^{2}-D\right)$ implies that the product of the $d$ transpositions $\theta\left(\gamma_{1}\right), \ldots, \theta\left(\gamma_{d}\right)$ in $S_{n}$ is also trivial, and the connectedness of the considered covering of $\mathbb{C P}^{2}$ implies that these transpositions act transitively on $\{1, \ldots, n\}$ and hence generate $S_{n}$.

It is a well-known fact that any two factorizations of the identity element in $S_{n}$ as a product of the same number of transpositions generating $S_{n}$ are equivalent by a succession of Hurwitz moves (this can be seen e.g. by comparing the two corresponding $n$-sheeted simple branched covers of $\left.\mathbb{C P}^{1}\right)$. Therefore, after a suitable reordering of the sheets of the covering $\pi: D \rightarrow \mathbb{C P}^{1}$ (which amounts to a global conjugation of the braid factorization), one may freely assume that the permutations $\theta\left(\gamma_{i}\right)$ are equal to certain predetermined transpositions. Our choice of transpositions in the case of the branch curve of $f_{k}$ will be made explicit in $\S 3.6$. 
3.2. The folding process. We now compute the braid monodromy of the curve $V_{2}^{\prime}\left(D_{k}\right)$, where $D_{k}$ is the branch curve of one of the stably quasiholomorphic maps $f_{k}$ given by Proposition 1 and $V_{2}^{\prime}$ is a generic perturbation of $V_{2}^{0}$ as in $\S 2.2$. The idea is to use Lemma 1 to reduce oneself to the easy case where $D_{k}$ is a union of $d=\operatorname{deg} D_{k}$ lines through a point in $\mathbb{C P}^{2}$. In that case, $V_{2}^{\prime}\left(D_{k}\right)$ becomes a union of $d$ conics through a point, and its braid monodromy can be computed explicitly. The result is the following:

Proposition 3. The braid factorization corresponding to the curve $V_{2}^{\prime}\left(D_{k}\right)$ is given by the formula

$$
\Delta_{2 d}^{2}=\left(\prod_{i=1}^{d-1} \prod_{j=i+1}^{d} Z_{i^{\prime} j^{\prime}}^{2}\right) \cdot \prod_{i=1}^{d} Z_{i i^{\prime}} \cdot F_{k} \cdot\left(\prod_{i=1}^{d-1} \prod_{j=i+1}^{d} Z_{i^{\prime} j^{\prime}}^{2}\right)^{2} \cdot \prod_{i=1}^{d} Z_{i i^{\prime}},
$$

or equivalently

$$
\Delta_{2 d}^{2}=\prod_{i=1}^{d} \hat{Z}_{i i^{\prime}} \cdot F_{k} \cdot\left(\prod_{i=1}^{d-1} \prod_{j=i+1}^{d} Z_{i^{\prime} j^{\prime}}^{2}\right)^{3} \cdot \prod_{i=1}^{d} Z_{i i^{\prime}}
$$

where $F_{k}$ is the image of the braid factorization for $D_{k}$ via the embedding of the braid group $B_{d}$ in $B_{2 d}$ obtained by considering a ball containing only the first $d$ points, and $\hat{Z}_{i i^{\prime}}$ is a half-twist along the following path :

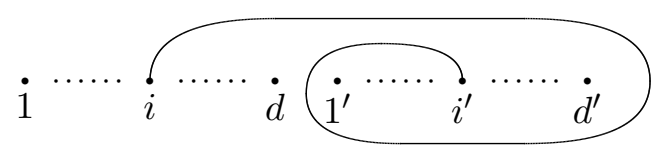

Equation (4) is an identity in the braid group $B_{2 d}$ acting on $2 d$ points labelled $1, \ldots, d, 1^{\prime}, \ldots, d^{\prime}$ (each pair $i, i^{\prime}$ corresponds to one of the $d$ conics).

Consider as in Lemma 1 the linear contraction map $\psi_{a}:(x: y: z) \mapsto(x:$ $a y+(1-a) x: a z+(1-a) x)$. When $a$ converges to 0 , the images of all the points outside of the line $L_{0}:\{x=0\}$ converge towards the point $p_{0}=(1: 1: 1)$. Since $\psi_{a}$ maps fibers of $\pi$ to fibers of $\pi$, the curves $\psi_{a}\left(D_{k}\right)$ are braided for all values of $a$. Moreover, $\psi_{a}$ restricts to the line $L_{0}:\{x=0\}$ as the identity, and when $a \rightarrow 0$ the image of any line intersecting $L_{0}$ transversely at a point $p=(0: y: z)$ converges to the line through $p$ and $p_{0}$.

By an arbitrarily small perturbation, and without losing the other properties of $D_{k}$, we can easily assume that the point $(0: 1: 1)$ does not belong to $D_{k}$, and that none of the nodes of $D_{k}$ lies on $L_{0}$. Therefore, by Lemma 1 the curve 
$V_{2}^{0}\left(\psi_{a}\left(D_{k}\right)\right)$ is locally braided for sufficiently small $a \neq 0$, and isotopic to $V_{2}^{\prime}\left(D_{k}\right)$ through locally braided curves. This implies that the braid factorizations for $V_{2}^{0}\left(\psi_{a}\left(D_{k}\right)\right)$ and for $V_{2}^{\prime}\left(D_{k}\right)$ are $m$-equivalent (in fact, when $D_{k}$ is a complex curve the isotopy can be carried out inside the complex category, so in that case the braid factorizations are even Hurwitz and conjugation equivalent).

When $a$ is sufficiently close to 0 , outside of a small ball centered at $p_{0}$ the curve $\psi_{a}\left(D_{k}\right)$ is arbitrarily close to the union of $d=\operatorname{deg} D_{k}$ lines joining the points of $D_{k} \cap L_{0}$ with $p_{0}$, and by construction the images by $V_{2}^{0}$ of these $d$ lines are distinct non-degenerate conics in $\mathbb{C P}^{2}$. Moreover, the restriction of $V_{2}^{0}$ to a neighborhood of $p_{0}$ is a diffeomorphism mapping fibers of $\pi$ to fibers of $\pi$. Therefore, the braid factorization of $V_{2}^{0}\left(\psi_{a}\left(D_{k}\right)\right)$, or equivalently that of $V_{2}^{\prime}\left(D_{k}\right)$ can be obtained by plugging the braid factorization of $D_{k}$ into the formula for the braid monodromy of a union of $d$ conics passing through the point $p_{0}$, i.e. by deleting a neighborhood of $p_{0}$ from this configuration and replacing it with a braided curve isotopic to the affine part of $D_{k}$ (suitably rescaled into a small ball).

As a first step, we therefore need to compute the braid monodromy of a union of $d$ conics passing through $p_{0}$. Observe that any configuration of $d$ non-degenerate conics in $\mathbb{C P}^{2}$ intersecting each other transversely at $p_{0}$ gives rise to a well-defined braid factorization as soon as none of the conics passes through the pole of the projection $\pi$ : any such configuration is a locally braided curve, and can be perturbed into a braided curve (a union of conics in general position) by an arbitrarily small perturbation. The connectedness of the space of configurations of conics implies that, up to Hurwitz and conjugation equivalence, it does not actually matter which conics are used for the computation of the braid monodromy.

Following Moishezon, the calculation can be carried out by simultaneously "degenerating" all the conics to pairs of lines, i.e. by considering a limit configuration where each of the conics is very close to a union of two lines [14]. However, for the purpose of proving Theorem 2 it is more efficient to perform a direct calculation using a specific configuration of conics. We consider $d$ conics with real coefficients, intersecting at the point $p_{0}$, and with their other mutual intersections lying close to three given points $p_{1}, p_{2}, p_{3}$, as in the following diagram (representing the intersection of the configuration with $\mathbb{R}^{2} \subset \mathbb{C}^{2}$, with the fibers of $\pi$ corresponding to vertical lines). 


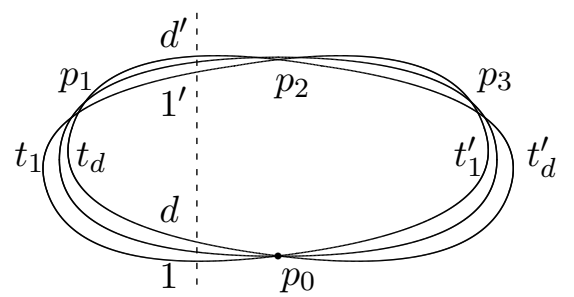

All the special points are sent to the real axis by the projection $\pi$; from left to right, there are $d$ tangency points $t_{1}, \ldots, t_{d}$, followed by $d(d-1) / 2$ nodes near $p_{1}$, the multiple point $p_{0}$, nodes near $p_{2}$, nodes near $p_{3}$, and finally $d$ tangency points $t_{1}^{\prime}, \ldots, t_{d}^{\prime}$. The base point is chosen on the real axis, immediately to the right of $\pi\left(p_{1}\right)$; the $d$ conics intersect the reference fiber of $\pi$ in $2 d$ points (all along the real axis in the fiber), labelled $1, \ldots, d, 1^{\prime}, \ldots, d^{\prime}$.

The system of generating loops that we use to define the braid factorization is given by paths joining the base point to the projections of the various tangencies and nodes as shown in the following diagram representing the base of the fibration $\pi:$

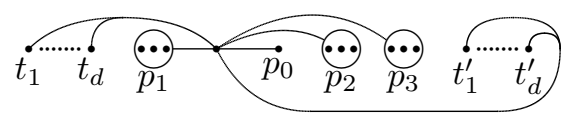

We order the various generating loops for $\pi_{1}(\mathbb{C}-$ crit $)$ counterclockwise around the base point, starting with the first of the arcs joining the base point to the projection of a node near $p_{1}$, and ending with the arc joining the base point to $\pi\left(t_{d}\right)$.

The contribution of each node or tangency point to braid monodromy can be calculated using a two-step process: first, one computes the local braid monodromy, i.e. the monodromy action on a fiber of $\pi$ very close to the critical point; this is the power of a half-twist exchanging two immediately adjacent intersection points of the considered fiber of $\pi$ with the configuration of $d$ conics. Next, the local configuration is brought back to the chosen fixed reference fiber of $\pi$ along a prescribed arc, and the desired braid is obtained as the image of the local monodromy under this "parallel transport" operation; for the purpose of the calculation, it is often efficient to perform a suitable homotopy in order to break down the given arc into a succession of half-circles centered on other critical 
values along the real axis, since parallel transport along such a half-circle can be explicitly described as a square root of the local monodromy.

The monodromy around the multiple intersection point $p_{0}$ is easily seen to be a full twist of a disc containing the $d$ intersection points labelled $1, \ldots, d$ in the reference fiber of $\pi$; we use the notation $\Delta_{d}^{2}$ for this element of $B_{2 d}$, which is actually the image of the central element $\Delta_{d}^{2} \in B_{d}$ under the natural embedding $B_{d} \hookrightarrow B_{2 d}$.

In the case of the nodes near $p_{1}$, the braid monodromy can be computed directly from the local picture; for a generic choice of the conics, the intersection points labelled $1^{\prime}, \ldots, d^{\prime}$ behave as in the case of $d$ lines in general position, and their $d(d-1) / 2$ intersections give rise to the braid monodromy factorization

$$
L_{d}^{\prime}=\prod_{i=1}^{d-1} \prod_{j=i+1}^{d} Z_{i^{\prime} j^{\prime}}^{2}
$$

or any Hurwitz equivalent expression (compare with equation (2)).

For the nodes near $p_{2}$, the local monodromy is the same as in the case of $p_{1}$, except that the ordering of the points $1, \ldots, d$ is reversed compared to the reference fiber of $\pi$ (these points are not affected by the local monodromy anyway). Since parallel transport along a half-circle around $p_{0}$ precisely amounts to a half-rotation of a disc containing the points labelled $1, \ldots, d$, the contribution to braid monodromy remains given by the same expression $L_{d}^{\prime}$ as above. Near $\pi\left(p_{3}\right)$, the local configuration is the same as for $p_{2}$ up to reversing the ordering of the points $1^{\prime}, \ldots, d^{\prime}$ inside the fibers of $\pi$; this discrepancy is taken care of by parallel transport along a half-circle centered at $p_{2}$, and so the contribution to braid monodromy is again $L_{d}^{\prime}$.

In the case of the tangency point $t_{d}$, the intersection of the $d$ conics with the fiber of $\pi$ above a point immediately to the right of $\pi\left(t_{d}\right)$ consists of $2 d$ points in the order $1, \ldots, d, d^{\prime}, \ldots, 1^{\prime}$ on the real axis, and the local monodromy is a halftwist exchanging the consecutive points $d, d^{\prime}$. Parallel transport along a clockwise half-circle around $\pi\left(p_{1}\right)$ induces a half-rotation of the disc containing $d^{\prime}, \ldots, 1^{\prime}$ in the clockwise direction, and therefore transforms this half-twist into $Z_{d d^{\prime}}$.

More generally, in a fiber immediately to the right of $t_{i}$, the local picture consists of $2 i$ points $1, \ldots, i, i^{\prime}, \ldots, 1^{\prime}$ on the real axis, while the points $d, \ldots,(i+$ $1),(i+1)^{\prime}, \ldots, d^{\prime}$ have moved to the pure imaginary axis, and the local monodromy 
around $t_{i}$ is a half-twist exchanging the consecutive points $i$ and $i^{\prime}$. Parallel transport along a clockwise half-circle around $\pi\left(t_{j}\right)$ for each $j>i$ rotates the two points $j$ and $j^{\prime}$ clockwise by $\frac{\pi}{2}$, which eventually yields the following half-twist in a fiber immediately to the right of $\pi\left(t_{d}\right)$ :

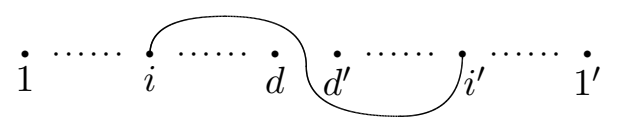

Finally, going around $\pi\left(p_{1}\right)$ we need to perform a clockwise half-rotation of a disc containing $d^{\prime}, \ldots, 1^{\prime}$, which yields the half-twist $Z_{i i^{\prime}}$ in the reference fiber of $\pi$. Therefore, the contribution of $t_{i}$ to the braid factorization is $Z_{i i^{\prime}}$.

The tangencies $t_{1}^{\prime}, \ldots, t_{d}^{\prime}$ are handled in the exactly the same manner; the calculations are slightly more tedious because of the more complicated choices of arcs joining $\pi\left(t_{i}^{\prime}\right)$ to the base point, but one easily checks that the braid monodromy around $t_{i}^{\prime}$ is again the half-twist $Z_{i i^{\prime}}$.

Putting the various contributions together in the correct order, we obtain that the braid monodromy for the chosen configuration of conics can be expressed by the factorization

$$
\Delta_{2 d}^{2}=L_{d}^{\prime} \cdot \prod_{i=1}^{d} Z_{i i^{\prime}} \cdot \Delta_{d}^{2} \cdot\left(L_{d}^{\prime}\right)^{2} \cdot \prod_{i=1}^{d} Z_{i i^{\prime}} .
$$

As explained at the beginning of this section, in order to get the braid factorization for $V_{2}^{\prime}\left(D_{k}\right)$ we need to replace in (5) the factor $\Delta_{d}^{2}$, corresponding to the local monodromy at the intersection point $p_{0}$, with the braid factorization corresponding to $D_{k}$, embedded into $B_{2 d}$ in the natural way by considering a disc containing the points $1, \ldots, d$ (see also the remark below). This immediately yields the formula (3).

The equivalent expression (4) is obtained from (3) by a sequence of Hurwitz moves, or equivalently, by a change in the choice of generators for $\pi_{1}(\mathbb{C}-$ crit $)$. Indeed, moving the factors in the first $L_{d}^{\prime}$ to the right across $\prod Z_{i i^{\prime}}$ and $F_{k}$ affects these latter factors by a conjugation by the inverse of the product of all the factors in $L_{d}^{\prime}$, i.e. by a clockwise full twist of the disc containing the points $1^{\prime}, \ldots, d^{\prime}$. As a result, $Z_{i i^{\prime}}$ is transformed into $\hat{Z}_{i i^{\prime}}$, while the factors in $F_{k}$ commute with those in $L_{d}^{\prime}$ and remain unaffected. This completes the proof of Proposition 3. 
Remark. As observed in $\S 1.1$, the braid factorization $F_{k}$ is only defined up to certain algebraic operations, among which global conjugation by an element of $B_{d}$. At first glance, the expressions obtained from (3) and (4) by replacing $F_{k}$ with its conjugate $\left(F_{k}\right)_{Q}$ by some braid $Q \in B_{d}$ appear to be inequivalent to the original unconjugated ones. Nonetheless, as suggested by the geometric intuition, all possible choices yield equivalent results for the braid factorization of $V_{2}^{\prime}\left(D_{k}\right)$. More precisely, defining $X_{r}=Z_{r, r+1}$ and $X_{r}^{\prime}=Z_{r^{\prime},(r+1)^{\prime}}$ for any $1 \leq r \leq d-1$, we claim that replacing $F_{k}$ by $\left(F_{k}\right)_{X_{r}}$ in the r.h.s. of (4) yields an expression which is Hurwitz and conjugation equivalent to the original one. This is proved by observing that the conjugated expressions $\left(L_{d}^{\prime}\right)_{X_{r}^{\prime}},\left(\prod \hat{Z}_{i i^{\prime}}\right)_{X_{r} X_{r}^{\prime}}$ and $\left(\prod Z_{i i^{\prime}}\right)_{X_{r} X_{r}^{\prime}}$ are Hurwitz equivalent to the unconjugated ones (checking these identities is an easy task left to the reader), so that a global conjugation by $X_{r} X_{r}^{\prime}$ and a sequence of Hurwitz moves can compensate for the conjugation of $F_{k}$.

3.3. The $V_{2}$ branch curve. We now compute the braid factorization corresponding to the branch curve $C_{2}$ of the quadratic map $V_{2}^{\prime}$ (or more generally of any generic quadratic holomorphic map from $\mathbb{C P}^{2}$ to itself). Elementary calculations show that $C_{2}$ is a curve of degree 6 with nine cusps, no nodal points, and tangent to the fibers of $\pi$ in three points.

The braid factorizations for branch curves of generic polynomial maps from $\mathbb{C P}^{2}$ to itself in any degree have been computed by Moishezon [15] (see also [16]), using a very technical and intricate argument. For the sake of completeness, we provide a direct calculation in the degree 2 case.

Proposition 4. The braid factorization for the branch curve of $V_{2}^{\prime}$ is given by the formula

(6) $\Delta_{6}^{2}=\left(Z_{13}^{3} Z_{14}^{3} Z_{12 ;(34)} Z_{23}^{3}\right) \cdot\left(Z_{15}^{3} Z_{16}^{3} Z_{12 ;(56)} Z_{25}^{3}\right) \cdot\left(Z_{35}^{3} Z_{36}^{3} Z_{34 ;(56)} Z_{45}^{3}\right)$, where $Z_{a b ;(c d)}=\left(Z_{b c}^{2} Z_{b d}^{2}\right) Z_{a b}\left(Z_{b c}^{2} Z_{b d}^{2}\right)^{-1}$ is a half-twist interchanging $a$ and $b$ along a path that goes around the points labelled $c$ and $d$, as follows:

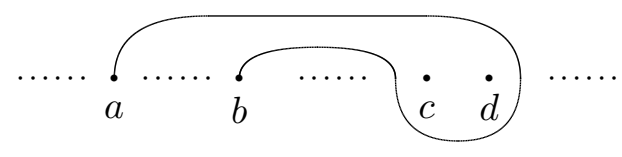

Proposition 4 is proved by studying the effect of a generic small deformation of the degenerate map $V_{2}^{0}:(x: y: z) \mapsto\left(x^{2}: y^{2}: z^{2}\right)$ on its branch curve. 
The ramification locus of $V_{2}^{0}$ in the source $\mathbb{C P}^{2}$ consists of three lines, which map two-to-one to three lines in the target $\mathbb{C P}^{2}$ : the branching divisor of $V_{2}^{0}$ therefore consists of three lines with multiplicity 2 (this behavior is extremely non-generic). The perturbation of $V_{2}^{0}$ into the generic map $V_{2}^{\prime}$ in particular affects the local behavior of the branch curve near the three points where the lines in the branch curve of $V_{2}^{0}$ intersect. It also affects the branch curve in a more global manner, since the multiplicity 2 lines making up the branch divisor of $V_{2}^{0}$ are deformed into a configuration without multiplicities; roughly speaking, away from the intersection points each line of multiplicity 2 is separated into two distinct lines lying close to each other (even though one must keep in mind that the curve $C_{2}$ is irreducible).

In order to avoid the pole of the projection $\pi$, we compose the map $V_{2}^{0}$ with the linear transformation $(x: y: z) \mapsto(x+\eta z: y+\eta z: z)$, for $\eta>0$ small. The resulting branch divisor still consists of three multiplicity 2 lines, intersecting the real slice $\mathbb{R}^{2} \subset \mathbb{C}^{2}$ in the following manner:

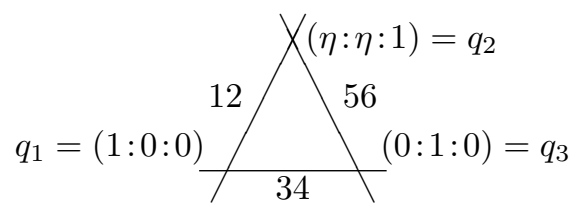

On this diagram, the fibers of $\pi$ correspond to vertical lines. We choose the reference fiber of $\pi$ far to the left on the real axis; after a generic perturbation, each of the three lines gives rise to two intersection points between $C_{2}$ and the reference fiber of $\pi$, for a total of 6 intersection points, all lying close to the real axis in the fiber. We label these points from 1 to 6 in the natural order along the real axis, namely we label 1 and 2 the two intersection points corresponding to the line $y=0$; we label 3 and 4 those corresponding to $z=0$, and finally 5 and 6 those corresponding to $x=0$.

The braid factorization is computed by considering the three intersection points, which obviously play very similar roles. The first intersection point $q_{1}$, for which we study the braid monodromy by considering paths close to the real axis in the base, involves the double lines $1-2$ and $3-4$, the first of which has the greatest slope ; computations in local coordinates yield a word in the braid group $B_{4}$, which needs to be embedded into $B_{6}$ simply by considering a disc containing the 
points 1,2,3,4 and centered on the real axis (the "parallel transport" operation is trivial in this case).

The second intersection point $q_{2}$ involves $1-2$ and $5-6$, the first of which again has the greatest slope ; because the local picture is the same, the local computation yields the same word in $B_{4}$ as for $q_{1}$. In the base of the fibration $\pi$, we choose to join $\pi\left(q_{2}\right)$ to the reference fiber via a path passing above the real axis; one easily checks that parallel transport along this path (going around $\pi\left(q_{1}\right)$ in the counterclockwise direction) amounts to a counterclockwise half-rotation of the points $1-2$ around $3-4$. As a consequence, we must now use an embedding of $B_{4}$ into $B_{6}$ corresponding to a domain containing the points $1,2,5,6$ and passing above the real axis near the points 3,4 . Finally, the third point $q_{3}$ involving $3-4$ and 5-6 again corresponds to the same local picture. We choose to join $\pi\left(q_{3}\right)$ to the base point by a path passing above the real axis, and one easily checks that, after parallel transport around $\pi\left(q_{1}\right)$ and $\pi\left(q_{2}\right)$, the relevant embedding of $B_{4}$ into $B_{6}$ is simply that given by a disc containing the points $3,4,5,6$ and centered on the real axis.

Consider any of the three intersection points $q_{1}, q_{2}, q_{3}$, where we want to compute the local contribution to braid monodromy after a small generic perturbation. Above such a point, the map $V_{2}^{0}$ is given in local affine coordinates by $(x, y) \mapsto\left(x^{2}, y^{2}\right)$; we choose to perturb it into the map

$$
f:(x, y) \mapsto\left(x^{2}+\alpha y, y^{2}+\beta x\right),
$$

where $\alpha$ and $\beta$ are small nonzero constants. The ramification curve is given by the vanishing of the Jacobian of $f$, which is $4 x y-\alpha \beta$; the branch curve of $f$ is therefore parametrized as

$$
\left\{\left(x^{2}+\frac{\alpha^{2} \beta}{4 x}, \frac{\alpha^{2} \beta^{2}}{16 x^{2}}+\beta x\right), x \in \mathbb{C}-\{0\}\right\} .
$$

We also need to specify the projection map in the local coordinates : it can be assumed to be $\left(z_{1}, z_{2}\right) \mapsto z_{1}+\epsilon z_{2}$ for a small nonzero value of the constant $\epsilon$.

With this setup, the branch curve of $f$ presents one tangency point and three cusps, and its braid monodromy can be determined explicitly by plotting the intersections of the branch curve with various fibers of the projection. The corresponding factorization in $B_{4}$ can be expressed as

$$
Z_{13}^{3} \cdot Z_{14}^{3} \cdot Z_{12 ;(34)} \cdot Z_{23}^{3}
$$


where $Z_{12 ;(34)}=\left(Z_{23}^{2} Z_{24}^{2}\right) Z_{12}\left(Z_{23}^{2} Z_{24}^{2}\right)^{-1}$ is the following half-twist :

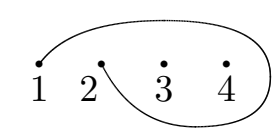

One can easily check that the product of the factors in (7) is equal to $Z_{12} Z_{34} Z_{13}^{2}$ $Z_{14}^{2} Z_{23}^{2} Z_{24}^{2}$, which amounts to the double lines $1-2$ and $3-4$ intersecting each other while the two lines in each double line ( 1 and 2 on one hand, 3 and 4 on the other hand) twist by a half-turn around each other : this is exactly the expected contribution (the presence of the half-twists is due to the fact that each double line is the image of a $2: 1$ covering branched at the singular point).

It is worth mentioning that the expression (7) is Hurwitz equivalent to its conjugates under the action of the half-twists $Z_{12}$ or $Z_{34}$ (or any combination of them). This "invariance property" is suggested by the geometric intuition, since the two points of each pair $1-2$ or $3-4$ arising from the perturbation of a double line play interchangeable roles; in fact, the diffeomorphisms $Z_{12}$ or $Z_{34}$ of the reference fiber of $\pi$ are induced by suitable changes in the parameters of the perturbation (from $\beta$ to $-\beta$ via $\left(e^{i \theta} \beta\right)_{0 \leq \theta \leq \pi}$, and similarly for $\alpha$, respectively). This observation explains why, although the three embeddings $B_{4} \rightarrow B_{6}$ described above are in fact naturally determined only up to certain conjugations, we need not worry about the lack of canonicality of our choices.

Finally, one obtains the braid factorization for $C_{2}$ by putting together the contributions of the three intersection points $q_{1}, q_{2}, q_{3}$, using the embeddings $B_{4} \rightarrow B_{6}$ described above. The images of (7) under these embeddings are exactly the three expressions appearing in the r.h.s. of (6). One easily checks that all the special points of $C_{2}$ are accounted for, either by using the Plücker formulas to show that $C_{2}$ only has 9 cusps and 3 tangency points, or by verifying directly that the product of the factors in the r.h.s. of (6) is equal to the central element $\Delta_{6}^{2}$. This completes the proof of Proposition 4 .

Let us point out that, although (7) looks very similar to the formula obtained by Moishezon for the braid monodromy at what he calls a "3-point" [13], the two geometric situations are very different : Moishezon's 3-points correspond to a generic projection of a very degenerate algebraic surface, with locally a covering map of degree 3 , while the points we describe here correspond to a very 
degenerate projection of a smooth algebraic surface, with locally a covering map of degree 4 . The fact that two very different geometric descriptions of the curve $C_{2}$ yield identical braid factorizations is one of the many remarkable properties of quadratic maps from $\mathbb{C P}^{2}$ to itself.

We finish this section by briefly describing the geometric monodromy representation $\theta_{V_{2}}: \pi_{1}\left(\mathbb{C P}^{2}-C_{2}\right) \rightarrow S_{4}$ corresponding to the factorization (6). Each double line in the branch curve of $V_{2}^{0}$ corresponds to two disjoint transpositions in $S_{4}$, while the transpositions corresponding to lines in different double lines are adjacent. Therefore, after a suitable reordering of the four sheets of the covering $V_{2}^{\prime}$, one may assume that the six geometric generators $\gamma_{1}, \ldots, \gamma_{6}$ (small loops going around each of the six points labelled $1, \ldots, 6$ in the reference fiber of $\pi$ ) are mapped to the transpositions (1 2), (3 4), (1 3), (2 4), (1 4) and (2 3) respectively. One easily checks that all the braids appearing in the factorization (6) satisfy the compatibility relations stated in the introduction (e.g., for the first factor $Z_{13}^{3}$, the transpositions $\theta_{V_{2}}\left(\gamma_{1}\right)=\left(\begin{array}{ll}1 & 2\end{array}\right)$ and $\theta_{V_{2}}\left(\gamma_{3}\right)=\left(\begin{array}{ll}1 & 3\end{array}\right)$ are indeed adjacent).

3.4. Regeneration of the mutual intersections. We now describe the contribution to the braid monodromy of $D_{2 k}$ of an intersection point of $V_{2}^{\prime}\left(D_{k}\right)$ with $C_{2}$. As observed in $\S 2.2$, the behavior of the map $f_{2 k}^{\prime}=V_{2}^{\prime} \circ f_{k}$ above such a point is not generic, and a perturbation is needed in order to obtain the generic map $f_{2 k}$. The local description of this perturbation is the following:

Lemma 2. Over a neighborhood of a point where $R_{k}$ intersects $f_{k}^{-1}\left(R_{2}^{\prime}\right)$, up to an isotopy of the branch curve among locally braided curves we can assume that $f_{2 k}^{\prime}$ and $f_{2 k}$ are given by the following models in local complex coordinates: $f_{2 k}^{\prime}(x, y)=\left(-x^{2}+y,-y^{2}\right)$, and $f_{2 k}(x, y)=\left(-x^{2}+y,-y^{2}+\epsilon x\right)$, where $\epsilon$ is a small non-zero constant, $\pi$ being the projection to the first component.

Proof. Provided that $k$ is large enough and given a point $p \in R_{k} \cap f_{k}^{-1}\left(R_{2}^{\prime}\right)$, the argument in $\S 3$ of [1] (see also [5],[3]) implies that a small perturbation term, localized near $p$, can be added to $f_{2 k}^{\prime}$ in order to make it generic and achieve the required transversality properties near $p$; the other transversality properties of $f_{2 k}^{\prime}$ are not affected if the perturbation is chosen small enough. Moreover, the one-parameter construction used in [1] to prove uniqueness up to isotopy implies that the space of admissible perturbations is path connected (once again provided that $k$ is large enough). 
Local models for the various maps can be obtained as follows. First observe that there exist local holomorphic coordinates $\left(z_{1}, z_{2}\right)$ on $\mathbb{C P}^{2}$ near $f_{k}(p)$ in which $V_{2}^{\prime}$ can be expressed as $\left(z_{1}, z_{2}\right) \mapsto\left(z_{1},-z_{2}^{2}\right)$. Moreover, it was shown in [1] that there exist local approximately holomorphic coordinates $(x, y)$ on $X$ and $\left(\tilde{z}_{1}, \tilde{z}_{2}\right)$ on $\mathbb{C P}^{2}$ in which $f_{k}$ is given by $(x, y) \mapsto\left(x^{2}, y\right)$.

Recall that $f_{k}$ satisfies properties (2) and (5) of Definition 7. Therefore, provided that $V_{2}^{\prime}$ is chosen sufficiently close to $V_{2}^{0}$ (which is always assumed to be the case), we know two things : first, by property (2), the branch curve $D_{k}=f_{k}\left(R_{k}\right)$ intersects the ramification curve $R_{2}^{\prime}$ of $V_{2}^{\prime}$ transversely ; second, by property (5), the tangent space to $D_{k}$ at $f_{k}(p)$ does not lie in the kernel of the differential of $V_{2}^{\prime}$, i.e. the image by $V_{2}^{\prime}$ of the branch curve of $f_{k}$ is locally immersed. Therefore, $D_{k}$, given by the equation $\tilde{z}_{1}=0$, is transverse at $f_{k}(p)$ to both axes of the coordinate system $\left(z_{1}, z_{2}\right)$ on $\mathbb{C P}^{2}$.

A first consequence is that $\left(\tilde{z}_{1}, z_{2}\right)$ are local approximately holomorphic coordinates on $\mathbb{C P}^{2}$; replacing the coordinate $y$ on $X$ by $\tilde{y}=f_{k}^{*}\left(z_{2}\right)$, we obtain that the expression of $f_{k}$ in the local coordinates $(x, \tilde{y})$ and $\left(\tilde{z}_{1}, z_{2}\right)$ remains $(x, y) \mapsto\left(x^{2}, y\right)$.

Another consequence is that the coefficients of $\tilde{z}_{1}$ and $z_{2}$ in the expression of $z_{1}$ as a function of $\tilde{z}_{1}$ and $z_{2}$ are both non-zero. Therefore, near the origin we can write $z_{1}=\tilde{z}_{1} \phi\left(\tilde{z}_{1}, z_{2}\right)+z_{2} \psi\left(z_{2}\right)+O\left(k^{-1 / 2}\right)$, where $\phi$ and $\psi$ are non-vanishing holomorphic functions and the last part corresponds to the antiholomorphic terms.

Working with coordinates $\left(z_{1}, z_{2}\right)$ on $\mathbb{C P}^{2}$, the expression of $f_{k}$ becomes $(x, y) \mapsto$ $\left(x^{2} \phi\left(x^{2}, y\right)+y \psi(y)+O\left(k^{-1 / 2}\right), y\right)$. Performing the coordinate change $(x, y) \mapsto$ $\left(i x \phi\left(x^{2}, y\right)^{1 / 2}, y\right)$ on $X$, we can reduce the model for $f_{k}$ to the simpler expression $(x, y) \mapsto\left(-x^{2}+y \psi(y)+O\left(k^{-1 / 2}\right), y\right)$. Decomposing $\psi$ into even and odd degree parts, we can write

$$
f_{2 k}^{\prime}(x, y)=\left(-x^{2}+y \psi_{0}\left(y^{2}\right)+y^{2} \psi_{1}\left(y^{2}\right)+O\left(k^{-1 / 2}\right),-y^{2}\right) .
$$

Composing with the coordinate change $(u, v) \mapsto\left(u+v \psi_{1}(-v), v \psi_{0}(-v)^{2}\right)$ on $\mathbb{C P}^{2}$, we reduce to $f_{2 k}^{\prime}(x, y)=\left(-x^{2}+y \psi_{0}\left(y^{2}\right)+O\left(k^{-1 / 2}\right),-y^{2} \psi_{0}\left(y^{2}\right)^{2}\right)$. Finally, the coordinate change $(x, y) \mapsto\left(x, y \psi_{0}\left(y^{2}\right)\right)$ on $X$ yields the expression $f_{2 k}^{\prime}(x, y)=$ $\left(-x^{2}+y+O\left(k^{-1 / 2}\right),-y^{2}\right)$. This expression differs from the desired one only by antiholomorphic terms, which are bounded by $O\left(k^{-1 / 2}\right)$ and therefore can be discarded without affecting the local braid monodromy computations. 
We know that for large enough $k$ the space of admissible asymptotically holomorphic local perturbations of $f_{2 k}^{\prime}$ near $p$ (i.e. perturbations satisfying the required uniform transversality properties) is path connected. Therefore, we are free to choose the perturbation which suits best our purposes ; fixing a constant $\epsilon \neq 0$, we set $f_{2 k}$ to be of the form $(x, y) \mapsto\left(-x^{2}+y,-y^{2}+\epsilon x\right)$. One easily checks that, provided that the chosen value of $\epsilon$ is bounded from below independently of $k$, this map locally satisfies all the required properties.

Concretely, this perturbation of $f_{2 k}^{\prime}$ can be performed in the same manner as in [1], by considering the very localized asymptotically holomorphic sections $s_{k, p}^{\text {ref }}$ of $L^{\otimes k}$ with exponential decay away from $p$ first introduced by Donaldson in [8]. It is easy to check that, by adding to one of the sections of $L^{\otimes 2 k}$ defining the covering map $f_{2 k}^{\prime}$ a small multiple of $x \cdot s_{2 k, p}^{\mathrm{ref}}$, where $x$ is the first coordinate function on $X$ near $p$, the map $f_{2 k}^{\prime}$ itself is affected by a perturbation which coincides at the first order with the desired one. In view of the local models, this is sufficient to ensure that the branch curve agrees with the prescribed one up to isotopy among locally braided curves, and hence to ensure that the braid monodromy is as desired. In fact, replacing the coefficient in front of $s_{2 k, p}^{\mathrm{ref}}$ by a suitable polynomial of higher degree in the coordinates, we can even make the perturbation of $f_{2 k}^{\prime}$ coincide with the desired one up to arbitrarily high order.

We finally consider the projection $\pi$ used to define braid monodromy. Recall that the various hypotheses made on $V_{2}^{\prime}$ and $f_{k}$ ensure that the branch curve of $V_{2}^{\prime}$ remains locally transverse to the fibers of $\pi$. Furthermore, over a neighborhood of the considered point, the tangent space to the branch curve of $f_{2 k}^{\prime}$ in $\mathbb{C P}^{2}$ remains very close to the direction determined by the branch curve of $V_{2}^{\prime}$ (in our local model, the first coordinate axis) ; an easy calculation shows that the same property remains true for $f_{2 k}$ (see also below). It follows that the local braid monodromy does not depend at all on choice of the projection $\pi$ as long as its fibers are locally transverse to the first coordinate axis. Therefore, performing if necessary an isotopy among locally braided curves by means of a suitable rotation, we can safely assume $\pi$ to be the projection to the first coordinate axis.

By Lemma 2 we know that the local braid monodromy of $f_{2 k}$ can be computed using for $f_{2 k}$ the local model

$$
(x, y) \mapsto\left(-x^{2}+y,-y^{2}+\epsilon x\right)
$$


where $\epsilon$ is a small non-zero constant. The Jacobian of this map is $4 x y-\epsilon$, and its branch curve can be parametrized as

$$
\left\{\left(-x^{2}+\frac{\epsilon}{4 x},-\frac{\epsilon^{2}}{16 x^{2}}+\epsilon x\right), x \in \mathbb{C}-\{0\}\right\} .
$$

The signs have been chosen in such a way that, taking $\epsilon$ along the positive real axis and taking the base point at a large negative real value of the first coordinate, the intersection of the branch curve with the reference fiber of $\pi$ consists of three points aligned along the real axis, the left-most one corresponding to the branch curve of $f_{k}$ while the two others correspond to the branch curve of $V_{2}^{\prime}$.

Projecting to the first component (or choosing any other generic projection), the only remarkable features of the branch curve near the origin are three cusps, and the corresponding braid factorization can be expressed as

$$
Z_{12}^{3} \cdot Z_{13}^{3} \cdot Z_{12 ;(3)}^{3},
$$

where the point labelled 1 corresponds to the branch curve of $f_{k}$ while the points labelled 2 and 3 correspond to the branch curve of $V_{2}^{\prime}$, and where $Z_{12 \text {;(3) }}=$ $Z_{23}^{2} Z_{12} Z_{23}^{-2}$ is a half-twist exchanging 1 and 2 along a path that goes around 3 :

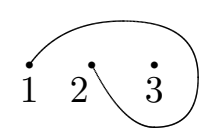

A short calculation in $B_{3}$ shows that the product of the factors in (8) is equal to $Z_{23}\left(Z_{12}^{2} Z_{13}^{2}\right)^{2}$, which amounts to the line labelled 1 twisting twice around 2 and 3 while these two lines undergo a half-twist. This is consistent with the geometric intuition, since the branch curve of $f_{k}$, folded onto itself by $V_{2}^{\prime}$, hits the branch curve of $V_{2}^{\prime}$ in a manner that can be represented by the following picture:

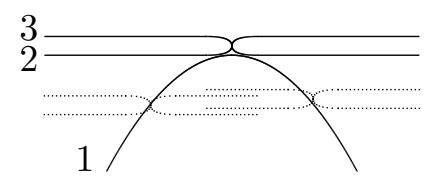

The line labelled 1 intersects 2 and 3 with multiplicity 2 because the image of $D_{k}$ by $V_{2}^{\prime}$ is necessarily tangent to the branch curve of $V_{2}^{\prime}$ wherever they intersect ; the lines 2 and 3 twist around each other by a half-turn because they arise as the 
two sheets of a 2:1 covering branched at the origin (they correspond to the two preimages by $f_{k}$ of each point where $V_{2}^{\prime}$ is ramified).

It is also worth observing that the expression (8) is easily shown to be Hurwitz equivalent to its conjugates under the action of the group generated by the halftwist $Z_{23}$ exchanging the two points labelled 2 and 3 , in agreement with the geometric intuition suggesting that their roles are interchangeable.

In order to understand how the braid monodromy given in (8) fits in the global picture, we now need to explain the labelling of the various components making up $D_{2 k}$ and the corresponding geometric monodromy representation.

Notations. As described above the branch curve $D_{2 k}$ is obtained by deforming the union of $V_{2}^{\prime}\left(D_{k}\right)$ and $n$ copies of $C_{2}$. Its degree is therefore $\bar{d}=2 d+6 n$. For braid group calculations in $B_{2 d+6 n}$, the $2 d+6 n$ intersection points of $D_{2 k}$ with the reference fiber of $\pi$ will be labelled as follows: we assign labels $1, \ldots, d$ and $1^{\prime}, \ldots, d^{\prime}$ to the $2 d$ intersection points which correspond to $V_{2}^{\prime}\left(D_{k}\right)$ (in the same manner as in $\S 3.2$ ), and $i_{\alpha}, i_{\alpha}^{\prime}, i_{\beta}, i_{\beta}^{\prime}, i_{\gamma}, i_{\gamma}^{\prime}$ for $1 \leq i \leq n$ to the $6 n$ intersection points corresponding to the $n$ copies of $C_{2}$. More precisely, recall that the branch curve of $V_{2}^{\prime}$ is obtained as a perturbation of the branch curve of $V_{2}^{0}$, which consists of three double lines : therefore the $n$ copies of $C_{2}$ can be thought of as three groups of $2 n$ lines. These three groups correspond to the three subscripts $\alpha, \beta$ and $\gamma$; for each value of $i$ the two labels $i_{\alpha}$ and $i_{\alpha}^{\prime}$ correspond to the perturbation of a double line in the $i$-th copy of the branch curve of $V_{2}^{0}$.

We will choose the reference fiber of $\pi$ and the configuration of the branch curve in such a way that the $2 d+6 n$ intersection points of the reference fiber with $D_{2 k}$ all lie on the real axis, in the order $1, \ldots, d, 1^{\prime}, \ldots, d^{\prime}, 1_{\alpha}, 1_{\alpha}^{\prime}, 2_{\alpha}, 2_{\alpha}^{\prime}, \ldots, n_{\alpha}, n_{\alpha}^{\prime}$, $1_{\beta}, 1_{\beta}^{\prime}, \ldots, n_{\beta}, n_{\beta}^{\prime}, 1_{\gamma}, 1_{\gamma}^{\prime}, \ldots, n_{\gamma}, n_{\gamma}^{\prime}$; in fact, we will actually choose a reference fiber yielding a slightly different configuration of intersection points, and then conjugate the obtained monodromy by a suitable braid. In any case, when using $Z_{i j}$ notations it will be understood that the $2 d+6 n$ intersection points of $D_{2 k}$ with the reference fiber of the projection $\pi$ are to be placed on the real axis in the above-given order.

In order to describe the geometric monodromy representation morphism $\theta_{2 k}$ : $\pi_{1}\left(\mathbb{C P}^{2}-D_{2 k}\right) \rightarrow S_{4 n}$, we first need to choose a set of geometric generators of $\pi_{1}\left(\mathbb{C P}^{2}-D_{2 k}\right)$. We choose the base point for $\pi_{1}\left(\mathbb{C P}^{2}-D_{2 k}\right)$ to lie in the reference 
fiber of $\pi$, far above the real axis which contains the $2 d+6 n$ intersection points with $D_{2 k}$, and we use a system of $2 d+6 n$ generating loops, each joining the base point to one of the intersection points along a straight line, circling once around the intersection point, and going back to the base point along the same straight line.

The $4 n$ sheets of the covering $f_{2 k}$ can be thought of as four groups of $n$ sheets, which we will label as $i_{a}, i_{b}, i_{c}, i_{d}$ for $1 \leq i \leq n$. Consider a situation similar to that of $\S 3.2$, where most of the branch curve of $f_{k}$ is concentrated into a small ball far away from the branch curve of $V_{2}^{\prime}$ : this results in a picture where the parts of the branch curve corresponding to $V_{2}^{\prime}\left(D_{k}\right)$ connect to each other the $n$ sheets of a single group $\left(1_{a}, \ldots, n_{a}\right.$ for example), while the copies of $C_{2}$ connect the various groups of $n$ sheets to each other. In particular, the transpositions in $S_{4 n}$ corresponding to the geometric generators around $1, \ldots, d, 1^{\prime}, \ldots, d^{\prime}$ are directly given by the geometric monodromy representation $\theta_{k}$ associated to $D_{k}$ : for any $1 \leq r \leq d$, if $\theta_{k}$ maps the $r$-th geometric generator to the transposition $(i j)$ in $S_{n}$ then, calling $\gamma_{r}$ and $\gamma_{r^{\prime}}$ the geometric generators in $\pi_{1}\left(\mathbb{C P}^{2}-D_{2 k}\right)$ corresponding to $r$ and $r^{\prime}$, one gets $\theta_{2 k}\left(\gamma_{r}\right)=\theta_{2 k}\left(\gamma_{r^{\prime}}\right)=\left(i_{a} j_{a}\right)$. Finally, each of the $n$ copies of $C_{2}$ connects four sheets to each other, one in each group of $n$, in the same manner as for $V_{2}^{\prime}$ itself : therefore $\theta_{2 k}$ maps the geometric generators around $i_{\alpha}, i_{\alpha}^{\prime}, i_{\beta}, i_{\beta}^{\prime}, i_{\gamma}$ and $i_{\gamma}^{\prime}$ to $\left(i_{a} i_{b}\right),\left(i_{c} i_{d}\right),\left(i_{a} i_{c}\right),\left(i_{b} i_{d}\right),\left(i_{a} i_{d}\right)$ and $\left(i_{b} i_{c}\right)$ respectively, for all $1 \leq i \leq n$.

A suitable choice of geometric configuration and reference fiber of $\pi$ yields a situation in which $\theta_{2 k}$ is as described above. Our choice of configuration will be made explicit in $§ 3.5$. A different set of geometric choices would lead to a different description of the braid monodromy and of $\theta_{2 k}$, but the final answer always remains the same up to Hurwitz and conjugation equivalence.

With this understood, we now describe the contribution to the braid monodromy of a point where a piece of $V_{2}^{\prime}\left(D_{k}\right)$, say e.g. the portion of conic labelled $r^{\prime}$ for some $1 \leq r \leq d$, hits one of the three groups of $2 n$ lines making up the $n$ copies of $C_{2}$, say e.g. the lines labelled $1_{\alpha}, 1_{\alpha}^{\prime}, \ldots, n_{\alpha}, n_{\alpha}^{\prime}$.

If one just considers the composed map $V_{2}^{\prime} \circ f_{k}$, the $n$ copies of the branch curve $C_{2}$ of $V_{2}^{\prime}$ all lie in the same position, and the curve $V_{2}^{\prime}\left(D_{k}\right)$ hits them tangently (and therefore with local intersection multiplicity +2 ). To obtain the generic map $f_{2 k}$ we add a small perturbation, which affects the situation by moving the 
$n$ copies of $C_{2}$ apart from each other and also by modifying the intersection of $R_{k}$ with $f_{k}^{-1}\left(R_{2}^{\prime}\right)$ in the manner explained above. More precisely, $R_{2}^{\prime}$ admits $2 n-2$ local lifts to $X$ which do not locally intersect the branch curve of $f_{k}$ (because they lie in different sheets of the covering) and thus do not require any special treatment, while the two other sheets of $f_{k}$ give rise to "lifts" of $R_{2}^{\prime}$ intersecting the branch curve of $f_{k}$ and each other. Therefore, when computing the braid factorization of $D_{2 k}$, we can locally consider the $n$ copies of $C_{2}$ as consisting of $2 n-2$ parallel lines, each intersected twice by $V_{2}^{\prime}\left(D_{k}\right)$ (giving rise to two nodes), and two "lines" parallel to the others which are hit by $V_{2}^{\prime}\left(D_{k}\right)$ in the manner previously explained.

The geometric monodromy representation $\theta_{2 k}$ maps the geometric generator around $r^{\prime}$ to a transposition of the form $\left(p_{a} q_{a}\right)$, for some $1 \leq p, q \leq n$. The two lines hit in a non-trivial manner are those labelled $p_{\alpha}$ and $q_{\alpha}$, which under the map $\theta_{2 k}$ correspond respectively to the transpositions $\left(p_{a} p_{b}\right)$ and $\left(q_{a} q_{b}\right)$ in $S_{4 n}$. The other $2 n-2$ lines ( $i_{\alpha}$ for $i \notin\{p, q\}$ and $i_{\alpha}^{\prime}$ for all $i$ ) lie in different sheets of the covering and their intersections with $r^{\prime}$ simply remain as nodes in the branch curve $D_{2 k}$.

Parallel transport of the local configuration along a given arc in the base of the fibration $\pi$ reveals the important role played by two specific paths in the reference fiber, namely the path along which the point labelled $r^{\prime}$ approaches the group of $2 n$ points $1_{\alpha}, \ldots, n_{\alpha}^{\prime}$ and the path along which two of these $2 n$ points approach each other. To phrase things differently, these two paths determine an embedded triangle with vertices at $r^{\prime}, p_{\alpha}, q_{\alpha}$ in the reference fiber, which collapses as one moves from the reference fiber towards the intersection point.

We assume the configuration to be such that, after parallel transport of the local configuration into the reference fiber of $\pi$, the path along which the point labelled $r^{\prime}$ approaches the $2 n$ other points is the simplest possible one passing above the real axis, while the two points $p_{\alpha}$ and $q_{\alpha}$ approach each other along a path isotopic to an arc contained in the upper half-plane. Equivalently, inside the reference fiber of $\pi$, we assume that the embedded triangle with vertices $r^{\prime}$, $p_{\alpha}$ and $q_{\alpha}$ which collapses as one approaches the considered singular point is the simplest possible one lying in the upper half-plane. Whether this is truly the case or whether the formula needs to be adjusted by a suitable global conjugation will be determined later on, when the contributions of the various points are put 
together into a global braid factorization in $B_{2 d+6 n}$; if the motions of $r^{\prime}, p_{\alpha}$ and $q_{\alpha}$ are different from the (purely arbitrary) above choice, then the formula giving the local monodromy will need to be conjugated by a certain element of $B_{2 d+6 n}$ (any braid that maps the triangle joining $r^{\prime}, p_{\alpha}, q_{\alpha}$ into the correct position can be used, as they all yield Hurwitz equivalent factorizations).

Proposition 5. The braid monodromy for the intersection of the portion of conic labelled $r^{\prime}$ with the $2 n$ lines $1_{\alpha}, 1_{\alpha}^{\prime}, \ldots, n_{\alpha}, n_{\alpha}^{\prime}$ is Hurwitz and conjugation equivalent to the following factorization:

(9) $\prod_{i=n}^{1}\left(\dot{Z}_{r^{\prime} i_{\alpha}^{\prime}}^{2}\left[\dot{Z}_{r^{\prime} i_{\alpha}}^{2}\right]_{i \notin\{p, q\}}\right) \cdot Z_{r^{\prime} p_{\alpha}}^{3} \cdot Z_{r^{\prime} q_{\alpha}}^{3} \cdot Z_{r^{\prime} p_{\alpha} ;\left(q_{\alpha}\right)}^{3}$.

$$
\prod_{i=n}^{1}\left(\grave{Z}_{r^{\prime} i_{\alpha}^{\prime}}^{2}\left[\grave{Z}_{r^{\prime} i_{\alpha}}^{2}\right]_{i \notin\{p, q\}}\right)
$$

where $\dot{Z}_{r^{\prime} \tau}$ and $\grave{Z}_{r^{\prime} \tau}$ are half-twists along the following paths:

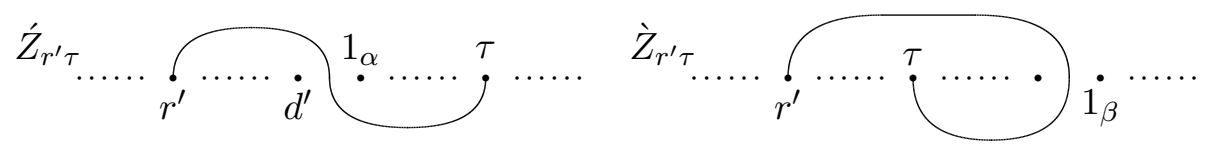

and $Z_{r^{\prime} p_{\alpha} ;\left(q_{\alpha}\right)}$ is a half-twist along the path

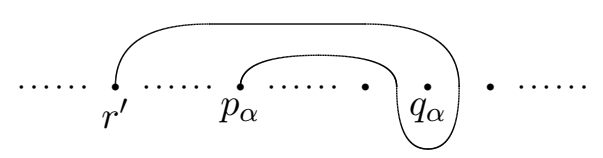

In (9), the products are to be performed in the reverse order (first $i=n$, finishing with $i=1)$, and the notation $[\ldots]_{i \notin\{p, q\}}$ means that the enclosed factor is not present for $i=p$ or $i=q$.

Proof. We start by considering a slightly simpler setup where, instead of being in their normal positions, the points $p_{\alpha}$ and $q_{\alpha}$ have been moved to the right of the $2 n-2$ other points $1_{\alpha}, \ldots, n_{\alpha}^{\prime}$ (i.e., further along the positive real axis in the reference fiber of $\pi$ ). More precisely, we assume that the points $p_{\alpha}$ and $q_{\alpha}$ have been moved into these positions along arcs in the upper half-plane, so that the point $r^{\prime}$ still reaches them by passing above the real axis and the vanishing 
cycle is the line segment joining $p_{\alpha}$ to $q_{\alpha}$. The situation is then described by the following picture in $\mathbb{R}^{2} \subset \mathbb{C}^{2}$ :

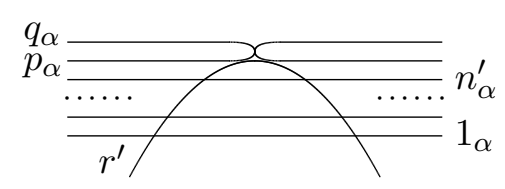

The reference fiber is once again placed to the left of the diagram, and the vertical direction corresponds to the real axis in the fibers of $\pi$.

Recalling that $V_{2}^{\prime}\left(D_{k}\right)$ hits $C_{2}$ tangently, the expected total contribution to the braid monodromy corresponds to $r^{\prime}$ twisting twice around each of the lines $1_{\alpha}, 1_{\alpha}^{\prime}, \ldots, n_{\alpha}, n_{\alpha}^{\prime}$. For the reasons explained above, a half-twist between the lines $p_{\alpha}$ and $q_{\alpha}$ is also to be expected.

In order to compute the braid monodromy, we observe that in the chosen configuration the singular fibers of $\pi$ all lie along the real axis, and choose the following system of generating paths in the base of the fibration $\pi$ : the first path connects the base point (far away on the negative real axis) to the first intersection of $r^{\prime}$ with $n_{\alpha}^{\prime}$ by passing below the real axis; the second one similarly joins the base point to the first intersection of $r^{\prime}$ with $n_{\alpha}$ by passing below the real axis; and so on, going from right to left, until all $2 n-2$ nodes in the left half of the diagram have been considered. The following three paths join the base point to the three cusp singularities arising from the perturbation of the singular point in the middle of the diagram, passing above the real axis. Finally, the remaining $2 n-2$ paths join the base point to the intersections in the right half of the diagram, passing above the real axis, and going from left to right (the first of these paths ends at the second intersection of $r^{\prime}$ with $n_{\alpha}^{\prime}$, the last one ends at the second intersection with $1_{\alpha}$ ). As should always be the case, the paths are ordered counterclockwise around the base point.

Observing that the conic labelled $r^{\prime}$ behaves similarly to the graph of the identity function in the left half of the diagram and similarly to the graph of -Id in the right half, one easily obtains the following expression for the local braid monodromy of our configuration:

$$
\prod_{i=n}^{1}\left(\dot{Z}_{r^{\prime} i_{\alpha}^{\prime}}^{2}\left[\dot{Z}_{r^{\prime} i_{\alpha}}^{2}\right]_{i \notin\{p, q\}}\right) \cdot F_{r^{\prime} p_{\alpha} q_{\alpha}} \cdot \prod_{i=n}^{1}\left(\grave{Z}_{r^{\prime} i_{\alpha}^{\prime}}^{2}\left[\grave{Z}_{r^{\prime} i_{\alpha}}^{2}\right]_{i \notin\{p, q\}}\right),
$$


where the notation $F_{r^{\prime}} p_{\alpha} q_{\alpha}$ represents an expression similar to (8), and $\dot{Z}_{r^{\prime} i_{\alpha}}$ and $\grave{Z}_{r^{\prime} i_{\alpha}}$ are the same half-twists as in the statement of Proposition 5.

We now bring the two points $p_{\alpha}$ and $q_{\alpha}$ back to their respective positions, moving them along paths passing above the real axis. The half-twists $\dot{Z}_{r^{\prime} \tau}$ and $\grave{Z}_{r^{\prime} \tau}$ are not affected by this motion; whereas $F_{r^{\prime} p_{\alpha} q_{\alpha}}$ is changed into $Z_{r^{\prime} p_{\alpha}}^{3} \cdot Z_{r^{\prime} q_{\alpha}}^{3}$. $Z_{r^{\prime} p_{\alpha} ;\left(q_{\alpha}\right)}^{3}$. Therefore, the expression (10) turns into (9).

Observe that the conjugates of the expression (9) by certain elements of $B_{2 n}$ (acting on $1_{\alpha}, \ldots, n_{\alpha}^{\prime}$ ) are Hurwitz equivalent to (9). Indeed, consider the subgroup $B_{2 n-2} \times B_{2} \subset B_{2 n}$ of braids which globally preserve the triangle formed by $r^{\prime}, p_{\alpha}$ and $q_{\alpha}$. The factor $B_{2}$ is generated by the half-twist $Z_{p_{\alpha} q_{\alpha}}$ interchanging $p_{\alpha}$ and $q_{\alpha}$, while the factor $B_{2 n-2}$ is generated by half-twists interchanging two of the $2 n-2$ other points along a path passing below the real axis. Conjugating (9) by $Z_{p_{\alpha} q_{\alpha}}$ simply amounts to a modification of the three central degree 3 factors of (9) by two Hurwitz moves. Similarly, conjugation by one of the half-twists generating $B_{2 n-2}$ (interchanging two consecutive points among the $2 n-2$ ) is equivalent to two Hurwitz moves, one among the $Z_{r^{\prime} \tau}^{2}$ factors and the other among the $\grave{Z}_{r^{\prime} \tau}^{2}$ factors. This is in agreement with the geometric intuition suggesting that, since all these conjugations do not affect the triangle joining $r^{\prime}, p_{\alpha}$ and $q_{\alpha}$, they do not modify the braid monodromy in any significant way.

However, as already pointed out above, conjugating (9) by an element of $B_{2 n}$ lying outside of $B_{2 n-2} \times B_{2}$ affects non-trivially the path along which $p_{\alpha}$ and $q_{\alpha}$ approach each other, and therefore yields an expression which is not Hurwitz equivalent to the original one (this can be seen directly by observing that the product of all factors in (9) is modified by the conjugation).

3.5. The assembling rule. We now study how the various elements described above fit together to provide the braid factorization for $D_{2 k}$. We will start by considering, as a toy model, a curve made up of $d$ conics and three lines, corresponding to the following diagram (drawn for $d=2$ ) : 


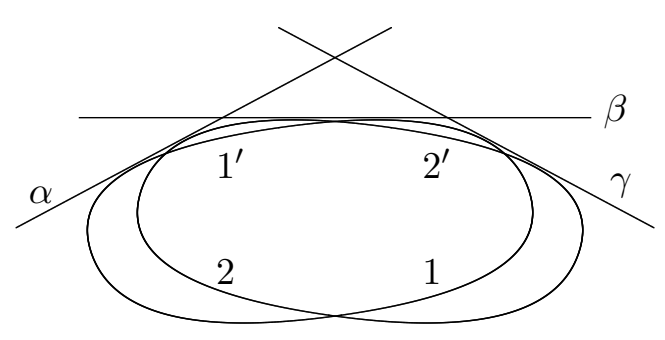

The $d$ conics play the role of $V_{2}^{\prime}\left(D_{k}\right)$, while the three lines correspond to the $C_{2}$ part. As usual, the vertical direction corresponds to the real axis in the fibers of $\pi$, and the reference fiber is to the left of the diagram ; in the reference fiber the points are placed on the real axis in the order $1, \ldots, d, 1^{\prime}, \ldots, d^{\prime}, \alpha, \beta, \gamma$. Although the space of all configurations of $d$ conics and three lines tangent to them in $\mathbb{C P}^{2}$ is connected, thus making all possible choices equally suitable, the choice of the configuration represented above is motivated by its remarkable similarity to the configurations chosen in $\S 3.2$ and $\S 3.3$ for $V_{2}^{\prime}\left(D_{k}\right)$ and $C_{2}$ respectively. In particular, one easily checks that the braid monodromy for the chosen configuration of the $d$ conics is exactly the one computed in $\S 3.2$ (equation (5) and Proposition $3)$.

The braid monodromy for this configuration of $d$ conics and three lines can be computed explicitly in coordinates. However this calculation is tedious and not very illuminating, so we first motivate the formula by deriving it by a different method : we start from a situation where the lines are in general position with respect to the conics, and we follow on the level of braid factorizations the deformation of such a generic configuration into the specific desired one. In fact, keeping track of the deformation amounts to performing a sequence of Hurwitz moves with the aim of bringing next to each other the two factors arising from the intersections of each line with each conic ; the resulting braid factorization contains consecutive identical degree 2 factors, so that merging the intersections becomes a trivial task.

Alternatively, the reader may jump ahead to the statement of Proposition 6 and the outline of proof given afterwards for a description of the direct monodromy calculation. 
The standard braid factorization assembling formula for the union of two tranversely intersecting curves of respective degrees $p$ and $q$ is given by

$$
\Delta_{p+q}^{2}=\Delta_{p}^{2} \cdot \prod_{i=1}^{p} \prod_{j=p+1}^{p+q} Z_{i j}^{2} \cdot \Delta_{q}^{2}
$$

where the points are labelled $1, \ldots, p$ for the first curve and $p+1, \ldots, p+q$ for the second, and $\Delta_{p}^{2}$ and $\Delta_{q}^{2}$ stand for the braid factorizations of the two components. The braid groups $B_{p}$ and $B_{q}$ are implicitly embedded into $B_{p+q}$ by considering two disjoint disks containing the $p$ first points and the $q$ last points respectively. The formula (11) can be easily checked by applying a suitable isotopy to the two components so that, outside of two mutually disjoint balls, they behave like respectively $p$ and $q$ mutually transverse lines.

In our case we want the three lines to be tangent to the conics, so we need to perform Hurwitz moves on this factorization so that the two intersections of each line with each conic can be brought together. Our starting point is the formula (11), together with a suitable braid monodromy factorization for a union of $d$ conics. For this we rely on Proposition 3, viewing the $d$ conics as the images of $d$ lines by a quadratic map; using the standard expression

$$
L_{d}=\prod_{i=1}^{d-1} \prod_{j=i+1}^{d} Z_{i j}^{2}
$$

for the braid monodromy of a configuration of $d$ lines, we obtain that the braid monodromy of a configuration of $d$ conics is given by substituting $L_{d}$ in the place of $F_{k}$ in equation (4). Thus the formula (11) gives the factorization

$$
\begin{aligned}
\Delta^{2}=\left(\prod_{i=1}^{d} \hat{Z}_{i i^{\prime}} \cdot L_{d} \cdot\left(L_{d}^{\prime}\right)^{3} \cdot \prod_{i=1}^{d} Z_{i i^{\prime}}\right) \cdot & \\
& \prod_{i=1}^{d}\left(Z_{i \alpha}^{2} Z_{i \beta}^{2} Z_{i \gamma}^{2}\right) \cdot \prod_{i=1}^{d}\left(Z_{i^{\prime} \alpha}^{2} Z_{i^{\prime} \beta}^{2} Z_{i^{\prime} \gamma}^{2}\right) \cdot\left(Z_{\alpha \beta}^{2} Z_{\alpha \gamma}^{2} Z_{\beta \gamma}^{2}\right) .
\end{aligned}
$$

Moving the $Z_{i i^{\prime}}$ factors to the right, one replaces the central $Z_{i \alpha}^{2} Z_{i \beta}^{2} Z_{i \gamma}^{2}$ terms by $Z_{i^{\prime} \alpha}^{2} Z_{i^{\prime} \beta}^{2} Z_{I^{\prime} \gamma}^{2}$; then, moving the rightmost terms to the left, one obtains the new expression

$$
\Delta^{2}=\left(\prod_{i=1}^{d} \hat{Z}_{i i^{\prime}} \cdot L_{d} \cdot\left(L_{d}^{\prime}\right)^{3}\right) \cdot\left(\prod_{i=1}^{d}\left(Z_{i^{\prime} \alpha}^{2} Z_{i^{\prime} \beta}^{2} Z_{i^{\prime} \gamma}^{2}\right)\right)^{2} \cdot\left(Z_{\alpha \beta}^{2} Z_{\alpha \gamma}^{2} Z_{\beta \gamma}^{2}\right) \cdot \prod_{i=1}^{d} \check{Z}_{i i^{\prime}}
$$


where $\check{Z}_{i i^{\prime}}$ is a half-twist along the following path :

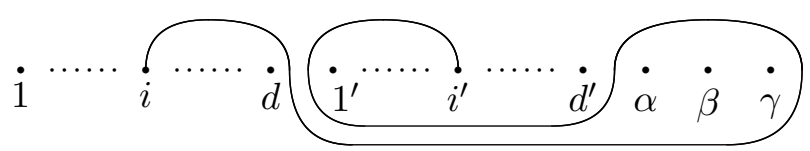

To shorten notations, we will write this factorization in the form

$$
\Delta^{2}=\prod_{i=1}^{d} \hat{Z}_{i i^{\prime}} \cdot L_{d} \cdot \Theta \cdot \prod_{i=1}^{d} \check{Z}_{i i^{\prime}}
$$

and work only with the central part $\Theta$, which geometrically corresponds to the upper half of the considered diagram. Using the commutativity rules in the central part, one can rewrite $\Theta$ as

$$
\Theta=\left(L_{d}^{\prime}\right)^{3} \cdot\left(\prod_{i=1}^{d} Z_{i^{\prime} \alpha}^{2} \prod_{i=1}^{d} Z_{i^{\prime} \beta}^{2} \prod_{i=1}^{d} Z_{i^{\prime} \gamma}^{2}\right)^{2} \cdot\left(Z_{\alpha \beta}^{2} Z_{\alpha \gamma}^{2} Z_{\beta \gamma}^{2}\right) .
$$

Moving the second set of $Z_{i^{\prime} \alpha}^{2}$ and $Z_{i^{\prime} \beta}^{2}$ factors to the left, one can rewrite this expression as

$$
\Theta=\left(L_{d}^{\prime}\right)^{3} \cdot\left(\prod_{i=1}^{d} Z_{i^{\prime} \alpha}^{2}\right)^{2} \cdot \prod_{i=1}^{d} Z_{i^{\prime} \beta ;\{\alpha\}}^{2} \cdot \prod_{i=1}^{d} Z_{i^{\prime} \beta}^{2} \cdot \prod_{i=1}^{d} Z_{i^{\prime} \gamma ;\{\beta\}}^{2} \cdot \prod_{i=1}^{d} Z_{i^{\prime} \gamma}^{2} \cdot\left(Z_{\alpha \beta}^{2} Z_{\alpha \gamma}^{2} Z_{\beta \gamma}^{2}\right)
$$

where $Z_{i^{\prime} \beta ;\{\alpha\}}$ and $Z_{i^{\prime} \gamma ;\{\beta\}}$ are half-twists along the following paths :
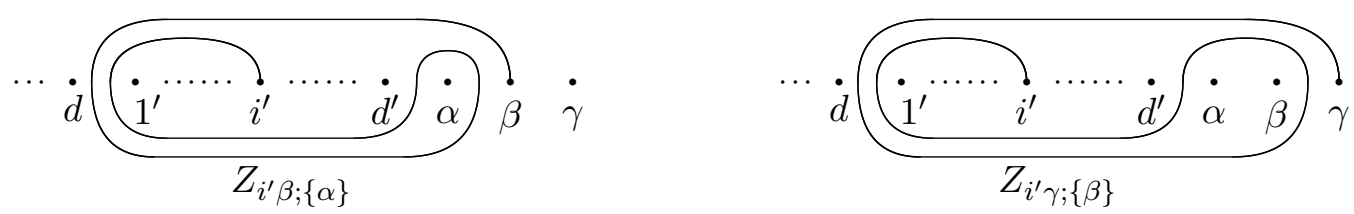

A succession of Hurwitz moves to the right makes it possible to rewrite $\Theta$ as

$$
\left(L_{d}^{\prime}\right)^{3} \cdot\left(\prod_{i=1}^{d} Z_{i^{\prime} \alpha}^{2}\right)^{2} \cdot \prod_{i=1}^{d} Z_{i^{\prime} \beta ;\{\alpha\}}^{2} \cdot \tilde{Z}_{\alpha \beta, 0}^{2} \cdot \prod_{i=1}^{d} Z_{i^{\prime} \beta}^{2} \cdot \prod_{i=1}^{d} Z_{i^{\prime} \gamma ;\{\beta\}}^{2} \cdot \tilde{Z}_{\alpha \gamma}^{2} \tilde{Z}_{\beta \gamma}^{2} \cdot \prod_{i=1}^{d} Z_{i^{\prime} \gamma}^{2}
$$

where $\tilde{Z}_{\alpha \beta, 0}, \tilde{Z}_{\alpha \gamma}$ and $\tilde{Z}_{\beta \gamma}$ are half-twists along the following paths :
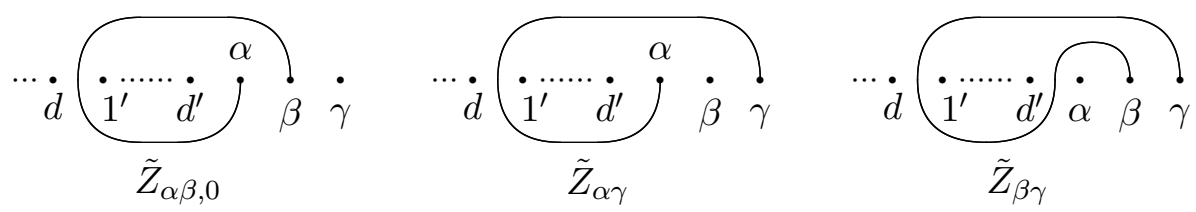
Moving $\tilde{Z}_{\alpha \beta, 0}^{2}, \tilde{Z}_{\alpha \gamma}^{2}$ and $\tilde{Z}_{\beta \gamma}^{2}$ to the left, one can rewrite $\Theta$ as

$$
\Theta=\left(L_{d}^{\prime}\right)^{3} \cdot\left(\prod_{i=1}^{d} Z_{i^{\prime} \alpha}^{2}\right)^{2} \cdot \tilde{Z}_{\alpha \beta, 0}^{2} \cdot\left(\prod_{i=1}^{d} Z_{i^{\prime} \beta}^{2}\right)^{2} \cdot \tilde{Z}_{\alpha \gamma}^{2} \tilde{Z}_{\beta \gamma}^{2} \cdot\left(\prod_{i=1}^{d} Z_{i^{\prime} \gamma}^{2}\right)^{2} .
$$

Moving the $Z_{i^{\prime} \beta}^{2}$ factors to the left, one obtains the new expression

$$
\Theta=\left(L_{d}^{\prime}\right)^{3} \cdot\left(\prod_{i=1}^{d} Z_{i^{\prime} \alpha}^{2}\right)^{2} \cdot\left(\prod_{i=1}^{d} Z_{i^{\prime} \beta}^{2}\right)^{2} \cdot \tilde{Z}_{\alpha \beta}^{2} \cdot \tilde{Z}_{\alpha \gamma}^{2} \tilde{Z}_{\beta \gamma}^{2} \cdot\left(\prod_{i=1}^{d} Z_{i^{\prime} \gamma}^{2}\right)^{2},
$$

where $\tilde{Z}_{\alpha \beta}$ is a half-twist along the path

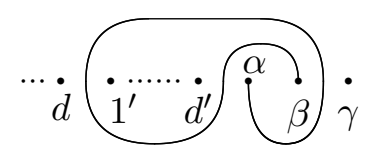

Observing that each factor $Z_{i^{\prime} j^{\prime}}^{2}$ in $L_{d}^{\prime}$ commutes with the products $\prod Z_{i^{\prime} \alpha}^{2}$ and $\prod Z_{i^{\prime} \beta}^{2}$ and also with $\tilde{Z}_{\alpha \beta}^{2}, \tilde{Z}_{\alpha \gamma}^{2}$ and $\tilde{Z}_{\beta \gamma}^{2}$, a sequence of Hurwitz moves to the left makes it possible to rewrite $\Theta$ as

$$
L_{d}^{\prime} \cdot\left(\prod_{i=1}^{d} Z_{i^{\prime} \alpha}^{2}\right)^{2} \cdot L_{d}^{\prime} \cdot\left(\prod_{i=1}^{d} Z_{i^{\prime} \beta}^{2}\right)^{2} \cdot \tilde{Z}_{\alpha \beta}^{2} \tilde{Z}_{\alpha \gamma}^{2} \tilde{Z}_{\beta \gamma}^{2} \cdot L_{d}^{\prime} \cdot\left(\prod_{i=1}^{d} Z_{i^{\prime} \gamma}^{2}\right)^{2} .
$$

We now study more in detail the first part of (14), namely

$$
\Theta_{\alpha}=L_{d}^{\prime} \cdot\left(\prod_{i=1}^{d} Z_{i^{\prime} \alpha}^{2}\right)^{2}=\prod_{i=1}^{d-1} \prod_{j=i+1}^{d} Z_{i^{\prime} j^{\prime}}^{2} \cdot\left(\prod_{i=1}^{d} Z_{i^{\prime} \alpha}^{2}\right)^{2} .
$$

A sequence of Hurwitz moves to the right makes it possible to rewrite this expression as

$$
\Theta_{\alpha}=\prod_{i=1}^{d-1} \prod_{j=i+1}^{d} Z_{i^{\prime} j^{\prime}}^{2} \cdot \prod_{i=1}^{d}\left(Z_{i^{\prime} \alpha}^{2} \hat{Z}_{i^{\prime} \alpha}^{2}\right),
$$

where $\hat{Z}_{i^{\prime} \alpha}$ is a half-twist along the path

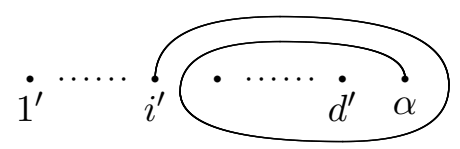


Using commutation relations, more Hurwitz moves yield the identity

$$
\Theta_{\alpha}=\prod_{i=1}^{d}\left(\prod_{j=i+1}^{d} Z_{i^{\prime} j^{\prime}}^{2} \cdot Z_{i^{\prime} \alpha}^{2} \hat{Z}_{i^{\prime} \alpha}^{2}\right) .
$$

Next we move $Z_{i^{\prime} \alpha}^{2}$ to the left and obtain

$$
\Theta_{\alpha}=\prod_{i=1}^{d}\left(Z_{i^{\prime} \alpha}^{2} \cdot \prod_{j=i+1}^{d} Z_{i^{\prime} j^{\prime} ;(\alpha)}^{2} \cdot \hat{Z}_{i^{\prime} \alpha}^{2}\right),
$$

where $Z_{i^{\prime} j^{\prime} ;(\alpha)}=Z_{i^{\prime} \alpha}^{-2} Z_{i^{\prime} j^{\prime}} Z_{i^{\prime} \alpha}^{2}$ is a twist along the path

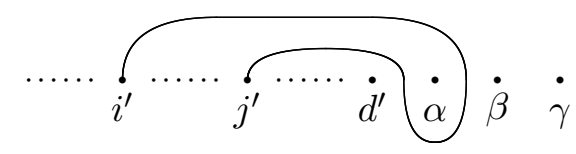

Finally, moving the $Z_{i^{\prime} j^{\prime} ;(\alpha)}^{2}$ factors to the left, one obtains the identity

$$
\Theta_{\alpha}=\prod_{i=1}^{d}\left(\left(Z_{i^{\prime} \alpha}^{2}\right)^{2} \cdot \prod_{j=i+1}^{d} Z_{i^{\prime} j^{\prime} ;(\alpha)}^{2}\right) .
$$

Geometrically this expression corresponds to the following picture :

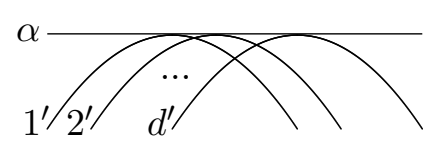

Proceeding similarly with the pieces involving $\beta$ and $\gamma$ in the expression (14), and letting $Z_{i^{\prime} j^{\prime} ;(\beta)}=Z_{i^{\prime} \beta}^{-2} Z_{i^{\prime} j^{\prime}} Z_{i^{\prime} \beta}^{2}$ and $Z_{i^{\prime} j^{\prime} ;(\gamma)}=Z_{i^{\prime} \gamma}^{-2} Z_{i^{\prime} j^{\prime}} Z_{i^{\prime} \gamma}^{2}$ (these twists correspond to the same picture as $Z_{i^{\prime} j^{\prime} ;(\alpha)}$ but going around $\beta$ or $\gamma$ instead of $\alpha$ ), the factorization (14) rewrites as

$$
\begin{aligned}
\Theta=\prod_{i=1}^{d}\left(\left(Z_{i^{\prime} \alpha}^{2}\right)^{2} \cdot \prod_{j=i+1}^{d} Z_{i^{\prime} j^{\prime} ;(\alpha)}^{2}\right) \cdot \prod_{i=1}^{d}\left(\left(Z_{i^{\prime} \beta}^{2}\right)^{2} \cdot \prod_{j=i+1}^{d} Z_{i^{\prime} j^{\prime} ;(\beta)}^{2}\right) \cdot \\
\tilde{Z}_{\alpha \beta}^{2} \tilde{Z}_{\alpha \gamma}^{2} \tilde{Z}_{\beta \gamma}^{2} \cdot \prod_{i=1}^{d}\left(\left(Z_{i^{\prime} \gamma}^{2}\right)^{2} \cdot \prod_{j=i+1}^{d} Z_{i^{\prime} j^{\prime} ;(\gamma)}^{2}\right) .
\end{aligned}
$$

We have finally achieved our goal of bringing next to each other the two intersections of each conic with each line. Therefore, going back to (13), we finally obtain : 
Proposition 6. The braid factorization corresponding to the union of $d$ conics and three lines tangent to them is given by

$$
\begin{array}{r}
\Delta^{2}=\prod_{i=1}^{d} \hat{Z}_{i i^{\prime}} \cdot L_{d} \cdot \prod_{i=1}^{d}\left(Z_{i^{\prime} \alpha}^{4} \cdot \prod_{j=i+1}^{d} Z_{i^{\prime} j^{\prime} ;(\alpha)}^{2}\right) \cdot \prod_{i=1}^{d}\left(Z_{i^{\prime} \beta}^{4} \cdot \prod_{j=i+1}^{d} Z_{i^{\prime} j^{\prime} ;(\beta)}^{2}\right) \cdot \\
\tilde{Z}_{\alpha \beta}^{2} \tilde{Z}_{\alpha \gamma}^{2} \tilde{Z}_{\beta \gamma}^{2} \cdot \prod_{i=1}^{d}\left(Z_{i^{\prime} \gamma}^{4} \cdot \prod_{j=i+1}^{d} Z_{i^{\prime} j^{\prime} ;(\gamma)}^{2}\right) \cdot \prod_{i=1}^{d} \check{Z}_{i i^{\prime}} .
\end{array}
$$

As explained above, the connectedness of the space of configurations of mutually tangent conics and lines implies that, for a different choice of the initial configuration, the braid factorization remains the same up to Hurwitz equivalence and global conjugation. For instance, using different expressions as starting points for the above geometric derivation of (15) leads to formulas in which the half-twists $\hat{Z}_{i i^{\prime}}$ and $\check{Z}_{i i^{\prime}}$ are replaced by slightly different half-twists with the same end points; these formulas are equivalent to (15) up to suitable Hurwitz moves and conjugations.

For completeness, we now describe how the reader may re-obtain the formula (15) by a direct calculation from the diagram presented at the beginning of this section (we describe the case $d=2$, the extension to all values of $d$ is trivial). We start again from the diagram representing the intersection of the configuration with $\mathbb{R}^{2} \subset \mathbb{C}^{2}$.

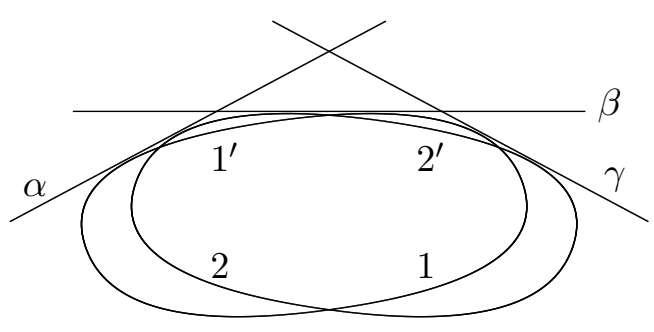

All the special points are sent to the real axis by the projection $\pi$, and labelling them in the obvious manner they are, from left to right, in the following order (after slightly deforming the projection in a manner which clearly doesn't affect the braid factorization) : $11^{\prime}, 22^{\prime}$ (tangencies), $12,1^{\prime} \alpha, 1^{\prime} 2^{\prime}, 2^{\prime} \alpha, \alpha \beta, 2^{\prime} \beta, 1^{\prime} 2^{\prime}, 1^{\prime} \beta$, $\alpha \gamma, \beta \gamma, 1^{\prime} \gamma, 1^{\prime} 2^{\prime}, 2^{\prime} \gamma$ (nodes and double nodes), 11', 22' (tangencies). (Note that, of the four intersections between the two conics, only one involves the portions 
labelled 1 and 2, while the three others involve the portions labelled $1^{\prime}$ and $2^{\prime}$; hence in the above list 12 appears only once and $1^{\prime} 2^{\prime}$ appears three times.)

The base point is placed on the real axis, immediately to the right of the first two tangencies (and to the left of all other points). The intersection with the reference fiber differs from the expected one by a permutation of the points labelled $1^{\prime}$ and $2^{\prime}$ (the points are in the order $1,2,2^{\prime}, 1^{\prime}, \alpha, \beta, \gamma$ ); this is taken care of by conjugating all computed monodromies by a half-twist, namely the point labelled $1^{\prime}$ is brought back to the left of $2^{\prime}$ by moving it counterclockwise along a half-circle passing above $2^{\prime}$.

The system of generating loops that we use to define the braid factorization is given by paths joining the base point to the various other points in the following manner (one easily checks that these paths are ordered counterclockwise around the base point). The first two paths join the base point to the points $11^{\prime}$ and $22^{\prime}$ on its left, starting below the real axis and rotating twice clockwise around $11^{\prime}$ and $22^{\prime}$ (see diagram below). The four following paths join the base point to the points $12,1^{\prime} \alpha, 1^{\prime} 2^{\prime}$ and $2^{\prime} \alpha$ on its right, passing above the real axis. The next four paths reach the points $1^{\prime} \beta, 1^{\prime} 2^{\prime}, 2^{\prime} \beta$ and $\alpha \beta$ in that order, starting above the real axis and crossing it between $1^{\prime} \beta$ and $\alpha \gamma$ to reach their end points from below, as shown on the diagram. The following two paths join the base point to $\alpha \gamma$ and $\beta \gamma$, simply passing above the real axis. The next three paths have $1^{\prime} \gamma$, $1^{\prime} 2^{\prime}$ and $2^{\prime} \gamma$ as end points, passing above the real axis but circling once clockwise around the three points before reaching them. Finally, the last two paths connect the base point to the two rightmost points $11^{\prime}$ and $22^{\prime}$, passing above the real axis and circling twice clockwise around them. The picture is as follows :

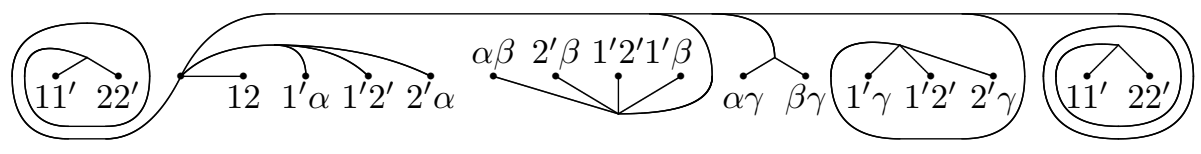

The monodromy around each point is computed using the following observation : placing oneself along the real axis, close to the image in the base of one of the special points, the intersection points of the curve with the fiber of $\pi$ all lie along the real axis (except at the outermost tangencies where some points have moved off the axis), and the two points involved in the monodromy lie next to each other. The monodromy then corresponds to a twist along a line segment between these two points ; more importantly, restricting oneself to a half-circle 
around the considered point in the base amounts to rotating the two points in the fiber around each other by half the total angle. With this understood, and decomposing each path into half-circles around the various points, the computations simply become a tedious task of careful accounting.

After suitably conjugating by a half-twist between $1^{\prime}$ and $2^{\prime}$, it turns out that the braid monodromies along the various given loops are exactly the factors appearing in (15), except in the case of the tangency points $11^{\prime}$ and $22^{\prime}$ at either extremity. In fact, the monodromies around the tangency points differ from $\hat{Z}_{i i^{\prime}}$ and $\check{Z}_{i i^{\prime}}$ by a conjugation by $Z_{12}^{4}$ (or more generally the square of $\Delta_{d}^{2}$ when $d>2$ ); a global conjugation of all factors by this braid eliminates the discrepancy and yields the desired formula.

(The choices made for the two sets of $d$ tangencies may seem rather artificial, and indeed other choices would lead in a slightly more direct manner to equally valid expressions - Hurwitz and conjugation equivalent to (15) - involving different half-twists instead of $\hat{Z}_{i i^{\prime}}$ and $\check{Z}_{i i^{\prime}}$. The choices made here are motivated by consistency with the geometric derivation outlined before the statement of Proposition 6, where these half-twists come up as a consequence of the use of Proposition 3 as a starting point for the calculation.)

3.6. The degree doubling formula. We finally turn to our main objective, computing the braid factorization for $D_{2 k}$. Recall from $\S 2.2$ that the generic covering map $f_{2 k}$ can be obtained as a small perturbation of $f_{2 k}^{\prime}=V_{2}^{\prime} \circ f_{k}$, where $V_{2}^{\prime}$ is a generic quadratic holomorphic map obtained by slightly perturbing $V_{2}^{0}:(x: y: z) \mapsto\left(x^{2}: y^{2}: z^{2}\right)$. More precisely, Proposition 2 states that, away from the intersection points of the two branch curves $R_{k}$ and $f_{k}^{-1}\left(R_{2}^{\prime}\right)$, the map $f_{2 k}^{\prime}$ satisfies almost all expected properties, the only problem for the definition of braid monodromy invariants being that its branch curve is not everywhere transverse to itself ; of course, it is also necessary to perturb $f_{2 k}^{\prime}$ near the intersection points in order to obtain a generic local model.

Recall that, by the main result of [5], $f_{2 k}^{\prime}$ can be made generic near the points of $\mathcal{I}_{k}^{\prime}=R_{k} \cap f_{k}^{-1}\left(R_{2}^{\prime}\right)$ by adding to it small perturbation terms (see also the argument at the end of $\S 2.2$ ). Provided that the perturbations are chosen small enough, the transversality properties satisfied by $f_{2 k}^{\prime}$ away from these points are not affected. Moreover, recall that for large $k$ the one-parameter argument proving the uniqueness up to isotopy of quasiholomorphic coverings also implies 
the connectedness of the space of admissible perturbations of $f_{2 k}^{\prime}$ near a given point of $\mathcal{I}_{k}^{\prime}$. Therefore, the perturbation of $f_{2 k}^{\prime}$ affects the braid monodromy near each point of $\mathcal{I}_{k}^{\prime}$ exactly as described in $\S 3.4$.

It is important to observe that these perturbations of $f_{2 k}^{\prime}$ only significantly affect the branch curve near the points of $\mathcal{I}_{k}^{\prime}$ : away from $\mathcal{I}_{k}^{\prime}$, the branch curve of the perturbed map remains $C^{1}$-close to that of $f_{2 k}^{\prime}$ (the perturbation terms are very small in comparison with the transversality estimates satisfied by $f_{2 k}^{\prime}$ ). Therefore, no unexpected changes can take place in the braid monodromy, although some pairs of nodes may be created when self-transversality is lost.

Another seemingly crucial point to be understood is the manner in which the $n$ copies of the branch curve of $V_{2}^{\prime}$ are moved into mutually transverse positions. Indeed, as explained at the end of $\S 3.4$ this information directly determines the contribution to the braid monodromy of the points of $\mathcal{I}_{k}^{\prime}$ by modifying the local configuration of vanishing cycles. Similarly, the braid monodromy arising near the points $(1: 0: 0),(0: 1: 0)$ and $(0: 0: 1)$ from the cusps and tangency points in the $n$ copies of $C_{2}$ is strongly related to the local configuration in each group of $2 n$ lines. Therefore, our lack of control over the manner in which each of the three groups of $2 n$ lines is arranged may seem rather disturbing.

Fortunately, up to $m$-equivalence this does not affect the final outcome of the calculations. Indeed, in most places the $2 n$ components labelled $1_{\alpha}, \ldots, n_{\alpha}^{\prime}$ (or similarly the two other groups of $2 n$ lines) all lift into different sheets of the covering $f_{2 k}: X \rightarrow \mathbb{C P}^{2}$; the only exceptions are near the intersection points of $C_{2}$ with $V_{2}^{\prime}\left(D_{k}\right)$, where two of the $2 n$ curves actually meet each other (e.g., those labelled $p_{\alpha}$ and $q_{\alpha}$ in $\S 3.4$ ), and similarly near the points of intersection between two groups of $2 n$ lines, where the two curves coming from the same copy of $C_{2}$ (e.g., those labelled $i_{\alpha}$ and $i_{\alpha}^{\prime}$ ) also merge. In any case, we are free to move the various lines across each other, as long as the two distinguished components are kept together ; in this process, the braid factorization only changes when pairs of intersections are created or cancelled, which always amounts to an $m$ equivalence. Observe moreover that all possible configurations can be deformed into each other in this way ; this follows e.g. from the fact that all the curves under consideration, whether self-transverse or not, are locally braided. We conclude that up to $m$-equivalence the braid monodromy does not depend on the chosen configuration. 
Another more algebraic way to express the same idea is the following. As observed at the end of $\S 3.4$, the manner in which the local braid monodromy arising from a point of $\mathcal{I}_{k}^{\prime}$ depends on the local configuration is a conjugation by an element $Q$ of $B_{2 n}$ which after multiplication by an element of $B_{2 n-2} \times B_{2}$ can easily be assumed to be a pure braid. Denoting by $\Phi$ the factorized expression corresponding to the standard configuration and by $\Phi_{Q}$ its conjugate by the braid $Q$, we have the chain of $m$-equivalences

$$
\Phi_{Q} \sim Q \cdot Q^{-1} \cdot \Phi_{Q} \sim Q \cdot \Phi \cdot Q^{-1} \sim Q \cdot Q_{\Phi}^{-1} \cdot \Phi,
$$

where the first operation is a pair creation and the two others are Hurwitz moves ; therefore, conjugating $\Phi$ by $Q$ is equivalent to inserting the two factors $Q$ and $Q_{\Phi}^{-1}$, which are both pure braids in $B_{2 n}$. A similar phenomenon occurs near the intersection points between two groups of $2 n$ lines : the choice of a specific configuration amounts to a conjugation by a pure braid in $B_{2 n} \times B_{2 n}$, which after a suitable $m$-equivalence simply amounts to inserting some pure braids into the factorization. Finally, some intersections between the $2 n$ lines also occur outside of these points, which means that, independently of the issue of the local configurations, some pure braids in $B_{2 n}$ appear as factors. Collecting all the pure braids in $B_{2 n}$ we have obtained in this description, we get that the choice of a specific configuration amounts to the choice of a set of pure braid factors in $B_{2 n}$ (or more precisely, three such sets of factors, one for each of the groups of lines labelled $\alpha, \beta$ and $\gamma$ ). The product of these factors is always the same independently of the chosen configuration, because in the end we only consider factorizations of $\Delta^{2}$. The result then follows from the following observation : given a pure braid $Q \in B_{2 n}$, any two decompositions of $Q$ into products of positive and negative twists differ from each other by Hurwitz moves and pair cancellations. This can be seen by realizing a factorization of $Q$ as the braid monodromy of a curve with $2 n$ components in $\mathbb{C}^{2}$ and by observing that any two such configurations are deformation equivalent. This is in fact a trivial case of the "symplectic isotopy problem", in which the components are sections of the projection, and the homotopy is allowed to create and cancel pairs of intersections between them. It is easy to convince oneself that for given $Q$ any two configurations are homotopic: for example, when $Q=1$ the components can be unknotted simply by translating them. See also [6] for a more general result. 
As explained in $\S 3.2$, we can deform the curve $D_{k}$ so that its image by $V_{2}^{0}$ becomes arbitrarily close to a union of $d$ conics, at which point the braid factorization for $V_{2}^{0}\left(D_{k}\right)$, or equivalently $V_{2}^{\prime}\left(D_{k}\right)$, is given by (4). First consider the singular map $V_{2}^{0} \circ f_{k}$, whose branch curve is the union of $V_{2}^{0}\left(D_{k}\right)$ with three lines (each of which has multiplicity $2 n$ ). These three lines always intersect $V_{2}^{0}\left(D_{k}\right)$ tangently. Therefore, after slightly deforming the map $V_{2}^{0}$ so that the three lines composing its branch curve avoid the pole of the projection $\pi$, the braid factorization for the branch curve of $V_{2}^{0} \circ f_{k}$ is very close to that given by Proposition 6 ; keeping in mind the result of $\S 3.2$, the only difference between the braid monodromy for $V_{2}^{0} \circ f_{k}$ and (15) is that the $L_{d}$ term in (15) should be replaced by the braid factorization $F_{k}$ for $D_{k}$.

The discussion at the beginning of this section gives a description of the modifications that occur when $V_{2}^{0}$ is replaced by $V_{2}^{\prime}$ and $f_{2 k}^{\prime}$ is perturbed into the generic map $f_{2 k}$. In this situation, the lines labelled $\alpha, \beta$ and $\gamma$ in $\S 3.5$ each need to be replaced by a set of $2 n$ lines. As we know from our study of the structure of $f_{2 k}$ near the points of $\mathcal{I}_{k}^{\prime}$, the factors $Z_{i^{\prime} \alpha}^{4}, Z_{i^{\prime} \beta}^{4}$ and $Z_{i^{\prime} \gamma}^{4}$ in (15) need to be replaced by expressions similar to $(9)$; as explained above we do not have to worry about the details of the local configurations.

Moreover, the factors $\tilde{Z}_{\alpha \beta}^{2}, \tilde{Z}_{\alpha \gamma}^{2}$ and $\tilde{Z}_{\beta \gamma}^{2}$ in (15) need to be replaced by the factorizations describing the behavior of $n$ copies of $C_{2}$ near one of the points where two groups of $2 n$ lines intersect each other. The contribution of each copy of $C_{2}$ has been computed in $\S 3.3$, but we must also take into account the mutual intersections between the various components. Fortunately, as explained above we do not have to worry about the exact local configuration, so we can choose one that simplifies calculations.

Finally, we also need consider the mutual intersections of the $2 n$ lines labelled $1_{\alpha}, \ldots, n_{\alpha}^{\prime}$ (and similarly in the two other groups) ; although the possibility of moving the lines across each other gives a lot of freedom, the manner in which they intersect is largely determined by the twisting phenomena arising at the points of intersection with $V_{2}^{\prime}\left(D_{k}\right)$ or with the other groups of $2 n$ lines. Indeed, since the total braid monodromy for the branch curve of $f_{2 k}$ has to be $\Delta^{2}$, the amount of twisting of any two lines around each other, and more precisely the product of all the degree \pm 2 factors involving $1_{\alpha}, \ldots, n_{\alpha}^{\prime}$, is entirely determined by the chosen configurations at the intersection points with $V_{2}^{\prime}\left(D_{k}\right)$ and the other 
groups of $2 n$ lines. As observed above, the various possible decompositions of this product into degree \pm 2 factors are all $m$-equivalent to each other, so that once again we can choose one freely (more geometrically, it is quite clear that any two configurations of the lines that are compatible with the local configurations chosen at the intersection points can be deformed into one another and hence yield $m$-equivalent results).

We now need to explicitly describe the geometric monodromy representation $\theta: F_{d} \rightarrow S_{n}$ for $f_{k}$. Recalling from $\S 3.1$ that all geometric morphisms $\theta: F_{d} \rightarrow S_{n}$ are equivalent to each other up to conjugation, we are free to choose the one most suited to our purposes ; since the choice that we now make is in some particular cases not the most practical one, we will also explain how to adapt the formula for a different choice of $\theta$.

Let us assume from now on that $n=\operatorname{deg} f_{k}$ and $d=\operatorname{deg} D_{k}$ satisfy the inequality $d \leq n(n-1)$. This inequality is satisfied in almost all examples ; in particular, given any symplectic 4 -manifold, it is satisfied as soon as $k$ is large enough. Consider geometric generators $\gamma_{1}, \ldots, \gamma_{d}$ of $\pi_{1}\left(\mathbb{C P}^{2}-D_{k}\right)$ : the loops $\gamma_{i}$ are contained in the reference fiber of the projection to $\mathbb{C P}^{1}$, in which, assuming that the base point and the $d$ intersection points with $D_{k}$ all lie on the real axis, they join the base point to the $i$-th intersection point by passing above the real axis, circle once counterclockwise around the intersection point, and return to the base point along the same path.

Performing a suitable global conjugation of the braid monodromy of $f_{k}$ if necessary, we can assume that the geometric monodromy representation is such that the transpositions $\theta\left(\gamma_{1}\right), \ldots, \theta\left(\gamma_{d}\right)$ are respectively equal to the $d$ first terms of the factorized expression

$$
\mathrm{Id}=\prod_{i=1}^{n-1} \prod_{j=i+1}^{n}(i j)(i j)
$$

in the symmetric group $S_{n}$. This choice is legal because $d$ is even and $d \geq 2 n-2$. For each $1 \leq i \leq n(n-1)$ we define the two indices $1 \leq p(i)<q(i) \leq n$ such that the $i$-th factor of this expression in $S_{n}$ is the transposition $(p(i) q(i))$; in particular $\theta\left(\gamma_{i}\right)=(p(i) q(i))$ for all $i \leq d$.

We first consider the contribution of the intersection points of $V_{2}^{\prime}\left(D_{k}\right)$ with $C_{2}$. Making the same choice of local configurations as in $\S 3.4$, each factor $Z_{i^{\prime} \alpha}^{4}$ in (15) 
needs to be replaced by

(16) $\begin{aligned} \prod_{j=n}^{1}\left(\dot{Z}_{i^{\prime} j_{\alpha}^{\prime}}^{2}\left[\dot{Z}_{i^{\prime} j_{\alpha}}^{2}\right]_{j \notin\{p(i), q(i)\}}\right) \cdot Z_{i^{\prime} p(i)_{\alpha}}^{3} \cdot Z_{i^{\prime} q(i)_{\alpha}}^{3} \cdot Z_{i^{\prime} p(i)_{\alpha} ;\left(q(i)_{\alpha}\right)^{3}}^{3} \\ \prod_{j=n}^{1}\left(\grave{Z}_{i^{\prime} j_{\alpha}^{\prime}}^{2}\left[\grave{Z}_{i^{\prime} j_{\alpha}}^{2}\right]_{j \notin\{p(i), q(i)\}}\right),\end{aligned}$

and similarly for the $Z_{i^{\prime} \beta}^{4}$ and $Z_{i^{\prime} \gamma}^{4}$ factors.

We next consider the intersections of the $2 n$ lines labelled $1_{\alpha}, \ldots, n_{\alpha}^{\prime}$ with the $2 n$ lines labelled $1_{\beta}, \ldots, n_{\beta}^{\prime}$. We choose as local configuration a situation consisting of $n$ identical copies of $C_{2}$ shifted away from each other by generic translations. The amounts by which the various copies are translated away from each other are assumed to be much larger than the distance between the two lines in a pair (e.g., $i_{\alpha}$ and $i_{\alpha}^{\prime}$ ); although this configuration can no longer be considered as a very small perturbation of $f_{2 k}^{\prime}$, it is quite clear that the translation process preserves the property of being locally braided, so that in terms of braid monodromy this configuration is $m$-equivalent to that obtained by a small perturbation of $f_{2 k}^{\prime}$. This choice of configuration can be represented on the following diagram :

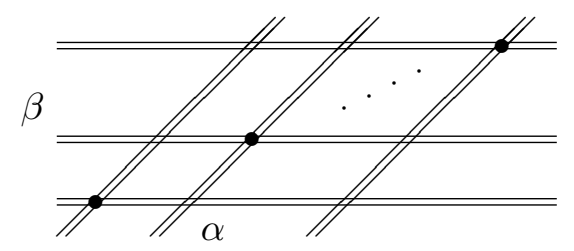

In this picture each intersection along the diagonal corresponds to a copy of $C_{2}$, yielding an expression similar to that in (7), while all other intersections occur between different copies of $C_{2}$ and simply yield nodes. However, recall from the computations in $\S 3.5$ that, when inserted into the expression for the global braid monodromy, all local braid monodromy contributions need to be conjugated in such a way that the various twists are performed along paths similar to the one appearing in the definition of $\tilde{Z}_{\alpha \beta}$. Therefore, if we momentarily ignore the specificities of the intersections along the diagonal, the braid monodromy for nodal intersections between the two sets of $2 n$ lines should be given by

$$
\prod_{i=1}^{n} \prod_{j=1}^{n}\left(\tilde{Z}_{i_{\alpha} j_{\beta}}^{2} \tilde{Z}_{i_{\alpha} j_{\beta}^{\prime}}^{2} \tilde{Z}_{i_{\alpha}^{\prime} j_{\beta}}^{2} \tilde{Z}_{i_{\alpha}^{\prime} j_{\beta}^{\prime}}^{2}\right) \text {, }
$$


where for any $\tau \in\left\{1_{\alpha}, 1_{\alpha}^{\prime}, \ldots, n_{\alpha}, n_{\alpha}^{\prime}\right\}$ and $v \in\left\{1_{\beta}, 1_{\beta}^{\prime}, \ldots, n_{\alpha}, n_{\beta}^{\prime}\right\}$ the notation $\tilde{Z}_{\tau v}$ represents a half-twist along the path

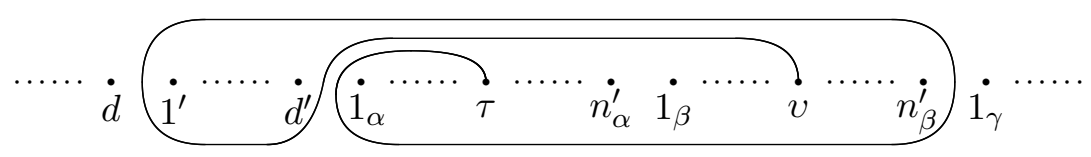

However, according to the calculations performed in $\S 3.3$, the intersections corresponding to $i=j$ consist of three cusps and one tangency point set up as in (7) rather than four nodes. Therefore, the correct contribution to the braid factorization of $f_{2 k}$ is given by the expression

$$
\begin{aligned}
\prod_{i=1}^{n}\left(\prod_{j=1}^{i-1}\left(\tilde{Z}_{i_{\alpha} j_{\beta}}^{2} \tilde{Z}_{i_{\alpha} j_{\beta}^{\prime}}^{2} \tilde{Z}_{i_{\alpha}^{\prime} j_{\beta}}^{2} \tilde{Z}_{i_{\alpha}^{\prime} j_{\beta}^{\prime}}^{2}\right) \cdot \tilde{Z}_{i_{\alpha} i_{\beta}}^{3} \tilde{Z}_{i_{\alpha} i_{\beta}^{\prime}}^{3} \tilde{Z}_{i_{\alpha} i_{\alpha}^{\prime} ;\left(i_{\beta} i_{\beta}^{\prime}\right)} \tilde{Z}_{i_{\alpha}^{\prime} i_{\beta}}^{3} \cdot\right. \\
\left.\prod_{j=i+1}^{n}\left(\tilde{Z}_{i_{\alpha} j_{\beta}}^{2} \tilde{Z}_{i_{\alpha} j_{\beta}^{\prime}}^{2} \tilde{Z}_{i_{\alpha}^{\prime} j_{\beta}}^{2} \tilde{Z}_{i_{\alpha}^{\prime} j_{\beta}^{\prime}}^{2}\right)\right),
\end{aligned}
$$

where $\tilde{Z}_{i_{\alpha} i_{\alpha}^{\prime} ;\left(i_{\beta} i_{\beta}^{\prime}\right)}=\left(\tilde{Z}_{i_{\alpha}^{\prime} i_{\beta}}^{2} \tilde{Z}_{i_{\alpha}^{\prime} i_{\beta}^{\prime}}^{2}\right) Z_{i_{\alpha} i_{\alpha}^{\prime}}\left(\tilde{Z}_{i_{\alpha}^{\prime} i_{\beta}}^{2} \tilde{Z}_{i_{\alpha}^{\prime} i_{\beta}^{\prime}}^{2}\right)^{-1}$ is a half-twist exchanging $i_{\alpha}$ and $i_{\alpha}^{\prime}$ along a path that goes around $i_{\beta}$ and $i_{\beta}^{\prime}$ (the $\alpha$ points being connected to the $\beta$ points along the same type of path described above).

The factors $\tilde{Z}_{\alpha \gamma}^{2}$ and $\tilde{Z}_{\beta \gamma}^{2}$ in (15) are treated similarly, and give rise to expressions similar to (17), except that the paths along which the $\tilde{Z}_{\tau v}^{2}$ factors twist now follow the model of $\tilde{Z}_{\alpha \gamma}^{2}$ or $\tilde{Z}_{\beta \gamma}^{2}$ instead of $\tilde{Z}_{\alpha \beta}^{2}$.

Our choice of local configuration for the $\alpha-\beta$ intersection is rather arbitrary ; however, a different choice would only affect the braid factorization by conjugation by a pure braid in $B_{2 n} \times B_{2 n}$ (each factor acting on one group of lines, while the path along which the groups are connected to each other necessarily remains that of $\tilde{Z}_{\alpha \beta}$ ). By the argument at the beginning of this section, such a conjugation amounts up to $m$-equivalence to inserting some pure braid factors in $B_{2 n} \times B_{2 n}$ into the global braid monodromy, which has been shown not to affect the outcome of the computations, so that we can safely ignore this issue.

We now look at the remaining nodal intersections between the $2 n$ lines $1_{\alpha}, 1_{\alpha}^{\prime}$, $\ldots, n_{\alpha}, n_{\alpha}^{\prime}$. The product of all these contributions to the braid monodromy is determined in the following manner by the previously chosen configurations at intersection points with $V_{2}^{\prime}\left(D_{k}\right)$ and with the other groups of $2 n$ lines. If we 
consider only the relative motions of the $2 n$ points labelled $1_{\alpha}, \ldots, n_{\alpha}^{\prime}$ induced by the various braids in the factorization, it is easy to check from the above formulas that the tangent intersection with the line labelled $i^{\prime}$ in $V_{2}^{\prime}\left(D_{k}\right)$ contributes a halftwist $Z_{p(i)_{\alpha} q(i)_{\alpha}}$ for all $1 \leq i \leq d$, while the intersection of $i_{\alpha}$ and $i_{\alpha}^{\prime}$ with $i_{\beta}$ and $i_{\beta}^{\prime}$ (or similarly $i_{\gamma}$ and $i_{\gamma}^{\prime}$ ) contributes the half-twist $Z_{i_{\alpha} i_{\alpha}^{\prime}}$. Therefore, the total contribution of intersection points is equal to $\prod_{i=1}^{d} Z_{p(i)_{\alpha} q(i)_{\alpha}} \cdot\left(\prod_{i=1}^{n} Z_{i_{\alpha} i_{\alpha}^{\prime}}\right)^{2}$.

On the other hand, recalling that we are looking for the braid factorization of a curve in $\mathbb{C P}^{2}$, the overall relative motions of the $2 n$ points $1_{\alpha}, \ldots, n_{\alpha}^{\prime}$ around each other must amount exactly to the central element $\Delta_{2 n}^{2}$ in $B_{2 n}$; the contribution of the additional nodal intersections is therefore exactly the difference between the contribution of intersection points and $\Delta_{2 n}^{2}$. Moreover, recall from the discussion at the beginning of this section that the decomposition of this contribution into a product of positive and negative twists is unique up to $m$-equivalence. In order to explicitly compute this decomposition, we first derive a suitable expression of $\Delta_{2 n}^{2}$. Viewing the $2 n$ points $1_{\alpha}, 1_{\alpha}^{\prime}, \ldots, n_{\alpha}, n_{\alpha}^{\prime}$ as $n$ groups of two points, it is easy to check that the full twist $\Delta_{2 n}^{2}$ can be expressed as

$$
\Delta_{2 n}^{2}=\prod_{i=1}^{n-1} \prod_{j=i+1}^{n}\left(Z_{i_{\alpha} j_{\alpha}}^{2} Z_{i_{\alpha} j_{\alpha}^{\prime}}^{2} Z_{i_{\alpha}^{\prime} j_{\alpha}}^{2} Z_{i_{\alpha}^{\prime} j_{\alpha}^{\prime}}^{2}\right) \cdot \prod_{i=1}^{n} Z_{i_{\alpha} i_{\alpha}^{\prime}}^{2} .
$$

Note that the two parts of this expression can be exchanged by Hurwitz moves. The second part of (18) corresponds exactly to the contribution of the intersection points with the two other groups of $2 n$ lines ; meanwhile, the first $d / 2$ factors $Z_{i_{\alpha} j_{\alpha}}^{2}$ correspond to the contribution of the points of $\mathcal{I}_{k}^{\prime}$ (recall the choice of geometric monodromy representation made above). Therefore, the nodal intersections correspond exactly to the remaining factors in (18). Inserting these braids at their respective positions in the factorization, and bringing the $\check{Z}_{i i^{\prime}}$ factors back to the beginning of the factorization by Hurwitz moves, we finally obtain the following result :

Theorem 3. Let $X$ be a compact symplectic 4-manifold, and let $f_{k}: X \rightarrow \mathbb{C P}^{2}$ be an approximately holomorphic branched covering given by three sections of $L^{\otimes k}$. Denote by $D_{k}$ the branch curve of $f_{k}$, and let $d=\operatorname{deg} D_{k}$ and $n=\operatorname{deg} f_{k}$. Assume that $d \leq n(n-1)$. Denote by $F_{k}$ the braid factorization corresponding to $D_{k}$, and assume that the geometric monodromy representation $\theta: \pi_{1}\left(\mathbb{C P}^{2}-D_{k}\right) \rightarrow S_{n}$ is as described at the beginning of §3.6, i.e. $\theta\left(\gamma_{i}\right)=(p(i) q(i))$. Then, with the notations described in $\S 3.4$, the braid factorization corresponding to the branch 
curve $D_{2 k}$ of $f_{2 k}$ is given up to $m$-equivalence by the following formula, provided that $k$ is large enough:

$$
\Delta_{2 d+6 n}^{2}=\mathbf{T}_{d} \cdot \iota\left(F_{k}\right) \cdot \mathbf{I}_{d, n, \theta}^{\alpha} \cdot \mathbf{I}_{d, n, \theta}^{\beta} \cdot \mathbf{V}_{n}^{\alpha \beta} \cdot \mathbf{V}_{n}^{\alpha \gamma} \cdot \mathbf{V}_{n}^{\beta \gamma} \cdot \mathbf{I}_{d, n, \theta}^{\gamma},
$$

where $\iota: B_{d} \hookrightarrow B_{2 d+6 n}$ is the natural embedding given by considering a disc containing the d leftmost points (labelled $1, \ldots, d$ ),

$$
\begin{aligned}
& \mathbf{T}_{d}=\prod_{i=1}^{d} \check{Z}_{i i^{\prime}} \cdot \prod_{i=1}^{d} \hat{Z}_{i i^{\prime}} \\
& \mathbf{I}_{d, n, \theta}^{\alpha}=\prod_{i=1}^{d}\left(\prod_{j=n}^{1}\left(\dot{Z}_{i^{\prime} j_{\alpha}^{\prime}}^{2}\left[Z_{i^{\prime} j_{\alpha}}^{2}\right]_{j \notin\{p(i), q(i)\}}\right) \cdot Z_{i^{\prime} p(i)_{\alpha}}^{3} \cdot Z_{i^{\prime} q(i)_{\alpha}}^{3} .\right. \\
& Z_{i^{\prime} p(i)_{\alpha} ;\left(q(i)_{\alpha}\right)}^{3} \cdot \prod_{j=n}^{1}\left(\grave{Z}_{i^{\prime} j_{\alpha}^{\prime}}^{2}\left[\grave{Z}_{i^{\prime} j_{\alpha}}^{2}\right]_{j \notin\{p(i), q(i)\}}\right) \cdot \prod_{j=i+1}^{d} Z_{i^{\prime} j^{\prime} ;(\alpha)}^{2} . \\
& \left.\left[Z_{p(i)_{\alpha} q(i)_{\alpha}^{\prime}}^{2} Z_{p(i)_{\alpha}^{\prime} q(i)_{\alpha}}^{2} Z_{p(i)_{\alpha}^{\prime} q(i)_{\alpha}^{\prime}}^{2}\right]_{i \equiv 0 \bmod 2}\right) \text {. } \\
& \prod_{i=(d / 2)+1}^{n(n-1) / 2}\left(Z_{p(2 i)_{\alpha} q(2 i)_{\alpha}}^{2} Z_{p(2 i)_{\alpha} q(2 i)_{\alpha}^{\prime}}^{2} Z_{p(2 i)_{\alpha}^{\prime} q(2 i)_{\alpha}}^{2} Z_{p(2 i)_{\alpha}^{\prime} q(2 i)_{\alpha}^{\prime}}^{2}\right) \text {, } \\
& \mathbf{I}_{d, n, \theta}^{\beta}=\prod_{i=1}^{d}\left(\prod_{j=n}^{1}\left(\dot{Z}_{i^{\prime} j_{\beta}^{\prime}}^{2}\left[\dot{Z}_{i^{\prime} j_{\beta}}^{2}\right]_{j \notin\{p(i), q(i)\}}\right) \cdot Z_{i^{\prime} p(i)_{\beta}}^{3} \cdot Z_{i^{\prime} q(i)_{\beta}}^{3} .\right. \\
& Z_{i^{\prime} p(i)_{\beta} ;\left(q(i)_{\beta}\right)}^{3} \cdot \prod_{j=n}^{1}\left(\grave{Z}_{i^{\prime} j_{\beta}^{\prime}}^{2}\left[\grave{Z}_{i^{\prime} j_{\beta}}^{2}\right]_{j \notin\{p(i), q(i)\}}\right) \cdot \prod_{j=i+1}^{d} Z_{i^{\prime} j^{\prime} ;(\beta)}^{2} . \\
& \left.\left[Z_{p(i)_{\beta} q(i)_{\beta}^{\prime}}^{2} Z_{p(i)_{\beta}^{\prime} q(i)_{\beta}}^{2} Z_{p(i)_{\beta}^{\prime} q(i)_{\beta}^{\prime}}^{2}\right]_{i \equiv 0 \bmod 2}\right) \text {. } \\
& \prod_{i=(d / 2)+1}^{n(n-1) / 2}\left(Z_{p(2 i)_{\beta} q(2 i)_{\beta}}^{2} Z_{p(2 i)_{\beta} q(2 i)_{\beta}^{\prime}}^{2} Z_{p(2 i)_{\beta}^{\prime} q(2 i)_{\beta}}^{2} Z_{p(2 i)_{\beta}^{\prime} q(2 i)_{\beta}^{\prime}}^{2}\right) \text {, }
\end{aligned}
$$




$$
\begin{aligned}
& \mathbf{I}_{d, n, \theta}^{\gamma}=\prod_{i=1}^{d}\left(\prod_{j=n}^{1}\left(\dot{Z}_{i^{\prime} j_{\gamma}^{\prime}}^{2}\left[\dot{Z}_{i^{\prime} j_{\gamma}}^{2}\right]_{j \notin\{p(i), q(i)\}}\right) \cdot Z_{i^{\prime} p(i)_{\gamma}}^{3} \cdot Z_{i^{\prime} q(i)_{\gamma}}^{3} .\right. \\
& Z_{i^{\prime} p(i)_{\gamma} ;\left(q(i)_{\gamma}\right)}^{3} \cdot \prod_{j=n}^{1}\left(\grave{Z}_{i^{\prime} j_{\gamma}^{\prime}}^{2}\left[\grave{Z}_{i^{\prime} j_{\gamma}}^{2}\right]_{j \notin\{p(i), q(i)\}}\right) \cdot \prod_{j=i+1}^{d} Z_{i^{\prime} j^{\prime} ;(\gamma)}^{2} . \\
& \left.\left[Z_{p(i)_{\gamma} q(i)_{\gamma}^{\prime}}^{2} Z_{p(i)_{\gamma}^{\prime} q(i)_{\gamma}}^{2} Z_{p(i)_{\gamma}^{\prime} q(i)_{\gamma}^{\prime}}^{2}\right]_{i \equiv 0 \bmod 2}\right) . \\
& \prod_{i=(d / 2)+1}^{n(n-1) / 2}\left(Z_{p(2 i)_{\gamma} q(2 i)_{\gamma}}^{2} Z_{p(2 i)_{\gamma} q(2 i)_{\gamma}^{\prime}}^{2} Z_{p(2 i)_{\gamma}^{\prime} q(2 i)_{\gamma}}^{2} Z_{p(2 i)_{\gamma}^{\prime} q(2 i)_{\gamma}^{\prime}}^{2}\right), \\
& \mathbf{V}_{n}^{\alpha \beta}=\prod_{i=1}^{n}\left(\prod_{j=1}^{i-1}\left(\tilde{Z}_{i_{\alpha} j_{\beta}}^{2} \tilde{Z}_{i_{\alpha} j_{\beta}^{\prime}}^{2} \tilde{Z}_{i_{\alpha}^{\prime} j_{\beta}}^{2} \tilde{Z}_{i_{\alpha}^{\prime} j_{\beta}^{\prime}}^{2}\right) \cdot \tilde{Z}_{i_{\alpha} i_{\beta}}^{3} \tilde{Z}_{i_{\alpha} i_{\beta}^{\prime}}^{3} \tilde{Z}_{\left.i_{\alpha} i_{\alpha}^{\prime} ; i_{\beta} i_{\beta}^{\prime}\right)} \tilde{Z}_{i_{\alpha}^{\prime} i_{\beta}}^{3} \cdot\right. \\
& \left.\prod_{j=i+1}^{n}\left(\tilde{Z}_{i_{\alpha} j_{\beta}}^{2} \tilde{Z}_{i_{\alpha} j_{\beta}^{\prime}}^{2} \tilde{Z}_{i_{\alpha}^{\prime} j_{\beta}}^{2} \tilde{Z}_{i_{\alpha}^{\prime} j_{\beta}^{\prime}}^{2}\right)\right) \\
& \mathbf{V}_{n}^{\alpha \gamma}=\prod_{i=1}^{n}\left(\prod_{j=1}^{i-1}\left(\tilde{Z}_{i_{\alpha} j_{\gamma}}^{2} \tilde{Z}_{i_{\alpha} j_{\gamma}^{\prime}}^{2} \tilde{Z}_{i_{\alpha}^{\prime} j_{\gamma}}^{2} \tilde{Z}_{i_{\alpha}^{\prime} j_{\gamma}^{\prime}}^{2}\right) \cdot \tilde{Z}_{i_{\alpha} i_{\gamma}}^{3} \tilde{Z}_{i_{\alpha} i_{\gamma}^{\prime}}^{3} \tilde{Z}_{i_{\alpha} i_{\alpha}^{\prime} ;\left(i_{\gamma} i_{\gamma}^{\prime}\right)} \tilde{Z}_{i_{\alpha}^{\prime} i_{\gamma}}^{3} .\right. \\
& \left.\prod_{j=i+1}^{n}\left(\tilde{Z}_{i_{\alpha} j_{\gamma}}^{2} \tilde{Z}_{i_{\alpha} j_{\gamma}^{\prime}}^{2} \tilde{Z}_{i_{\alpha}^{\prime} j_{\gamma}}^{2} \tilde{Z}_{i_{\alpha}^{\prime} j_{\gamma}^{\prime}}^{2}\right)\right) \\
& \mathbf{V}_{n}^{\beta \gamma}=\prod_{i=1}^{n}\left(\prod_{j=1}^{i-1}\left(\tilde{Z}_{i_{\beta} j_{\gamma}}^{2} \tilde{Z}_{i_{\beta} j_{\gamma}^{\prime}}^{2} \tilde{Z}_{i_{\beta}^{\prime} j_{\gamma}}^{2} \tilde{Z}_{i_{\beta}^{\prime} j_{\gamma}^{\prime}}^{2}\right) \cdot \tilde{Z}_{i_{\beta} i_{\gamma}}^{3} \tilde{Z}_{i_{\beta} i_{\gamma}^{\prime}}^{3} \tilde{Z}_{i_{\beta} i_{\beta}^{\prime} ;\left(i_{\gamma} i_{\gamma}^{\prime}\right)} \tilde{Z}_{i_{\beta}^{\prime} i_{\gamma}}^{3}\right. \\
& \left.\prod_{j=i+1}^{n}\left(\tilde{Z}_{i_{\beta} j_{\gamma}}^{2} \tilde{Z}_{i_{\beta} j_{\gamma}^{\prime}}^{2} \tilde{Z}_{i_{\beta}^{\prime} j_{\gamma}}^{2} \tilde{Z}_{i_{\beta}^{\prime} j_{\gamma}^{\prime}}^{2}\right)\right)
\end{aligned}
$$

In these expressions, the notation $[\ldots]_{i \equiv 0}$ mod 2 means that the enclosed factors are only present for even values of $i$; the notations $p(i)_{\alpha}, p(i)_{\alpha}^{\prime}$, and so on represent the labels $j_{\alpha}, j_{\alpha}^{\prime}$, etc where $j=p(i) \in\{1, \ldots, n\}$; and the various notations for braids correspond to half-twists along the following paths : 


$$
\begin{aligned}
& Z_{\tau v} \ldots \ldots \overbrace{\tau} \ldots \ldots \downarrow_{v} \ldots \ldots
\end{aligned}
$$

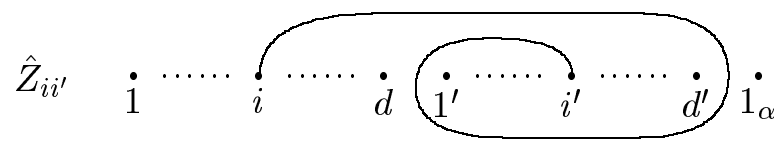

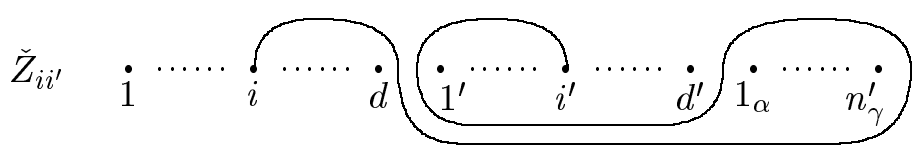

$$
\begin{aligned}
& Z_{i^{\prime} \tau ;(v)} \cdots \cdots \cdots \overbrace{i^{\prime}} \cdots \cdots+\overbrace{\tau} \ldots \cdots \cdot
\end{aligned}
$$

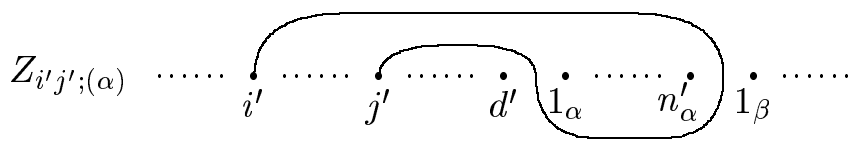

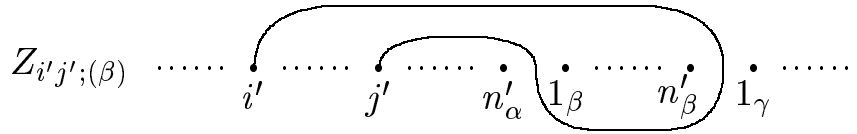

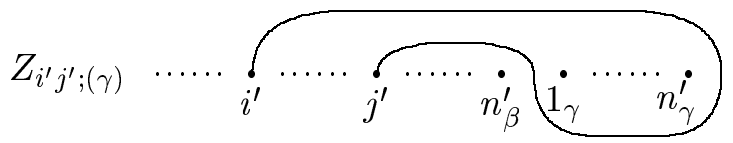

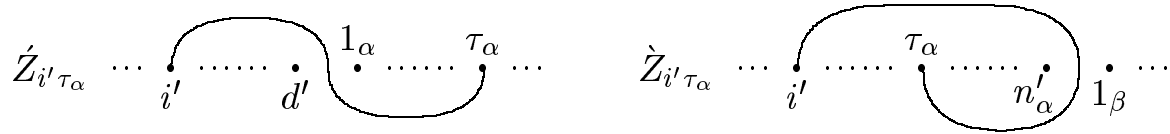

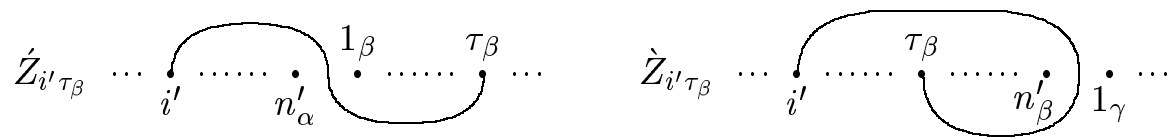

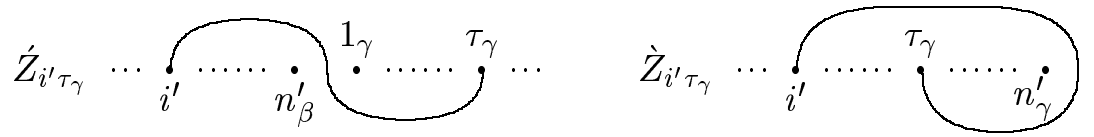




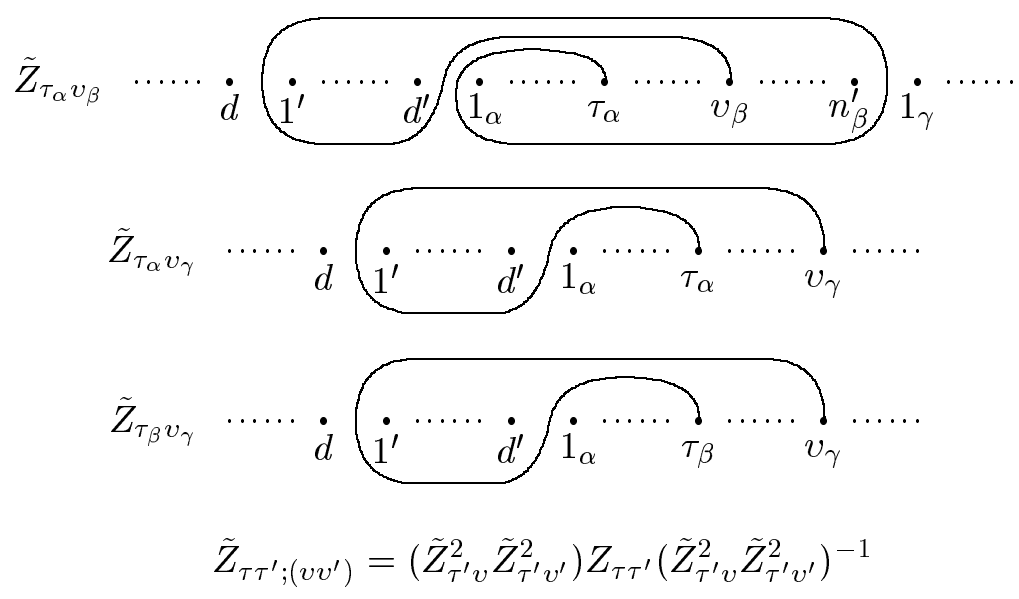

Remark : in the expression (19) we have made use of our specific choice of geometric monodromy representation for $f_{k}$, which requires the inequality $d \leq$ $n(n-1)$ to hold in counterpart for the relative simplicity of the resulting factors. Also, we have chosen to insert some of the pure braid factors involving the $2 n$ lines $1_{\alpha}, \ldots, n_{\alpha}^{\prime}$ amid the contributions of the intersection points of these lines with $V_{2}^{\prime}\left(D_{k}\right)$, in order to avoid the need for a rewriting of (18) using Hurwitz moves to isolate these contributions.

In general, if one wishes to get rid of the assumption made on the structure of the geometric monodromy representation $\theta$ and to remove the constraint $d \leq$ $n(n-1)$, the necessary modifications are rather easy and only involve finding a different expression of $\Delta_{2 n}^{2}$ to replace (18). Namely, denote by $(\tau(i) v(i)) \in S_{n}$ the image by $\theta$ of the $i$-th geometric generator of $\pi_{1}\left(\mathbb{C P}^{2}-D_{k}\right)$ (in the standard situation of (19) one has $\tau(i)=p(i)$ and $v(i)=q(i)$ but we now want to lift this assumption). Then, if we keep our choice of the simplest local geometric configurations at points of $\mathcal{I}_{k}^{\prime}$, the contribution of these points to the twisting among the lines $1_{\alpha}, \ldots, n_{\alpha}^{\prime}$ is given by the pure braid $\prod_{i=1}^{d} Z_{\tau(i)_{\alpha} v(i)_{\alpha}}$. We know that the total contribution of nodal intersections between the $2 n$ lines must be equal to

$$
Q_{\alpha}=\left(\prod_{i=1}^{d} Z_{\tau(i)_{\alpha} v(i)_{\alpha}}\right)^{-1} \cdot \Delta_{2 n}^{2} \cdot\left(\prod_{i=1}^{n} Z_{i_{\alpha} i_{\alpha}^{\prime}}^{2}\right)^{-1} .
$$

Since $Q_{\alpha}$ is a pure braid it can be decomposed into a product of positive and negative twists involving $1_{\alpha}, \ldots, n_{\alpha}^{\prime}$. The resulting modification of the factors in $\mathbf{I}_{d, n, \theta}^{\alpha}$ is as follows : in the first two lines, $p(i)$ and $q(i)$ should be replaced by 
$\tau(i)$ and $v(i)$ respectively ; the third line, consisting only of nodal intersections inserted amid the other contributions, should be deleted ; and the last line, containing the main group of nodal intersections, should be replaced by the chosen factorization of $Q_{\alpha}$. Similar modifications are also required in $\mathbf{I}_{d, n, \theta}^{\beta}$ and $\mathbf{I}_{d, n, \theta}^{\gamma}$.

As explained previously, the independence of the braid factorization upon the choice of local configurations and the fact that any two geometric monodromy representations differ from each other by a global conjugation imply that the expression obtained for a non-standard choice of $\theta$ is m-equivalent to the standard one. In particular, the possible presence of negative twists in the factorization of $Q_{\alpha}$ should not be considered as an indication of the existence of non-removable negative nodes.

Remark : when $X$ is a complex projective manifold, braid monodromy becomes well-defined up to Hurwitz equivalence and global conjugation only, since no negative nodes may appear in the (holomorphic) branch curve. However, (19) only gives the answer up to $m$-equivalence even in this case. If one looks more closely, the deformation process described in $\S 3.2$ can be performed algebraically provided that $L^{\otimes k}$ is sufficiently positive, and therefore remains valid in the complex setting ; in fact, all the braid monodromy computations described in $\S \S 3.2-$ 3.5 are valid not only up to $m$-equivalence but also up to Hurwitz equivalence and conjugation. However, what is not clear from an algebraic point of view is the exact configuration in which the lines $1_{\alpha}, \ldots, n_{\alpha}^{\prime}$ are placed by a generic algebraic perturbation performed near the points of $\mathcal{I}_{k}^{\prime}$. Determining this information now becomes an important matter, since our argument to show that all possible configurations are $m$-equivalent involves cancelling pairs of nodal intersections.

More precisely, provided that $d \leq n(n-1)$, by applying formula (19) we obtain a braid factorization without negative twists, which is $m$-equivalent to the braid factorization describing a generic algebraic map in degree $2 k$, but we don't know for sure whether the $m$-equivalence can be realized without creating pairs of nodal intersections between the $2 n$ lines $1_{\alpha}, \ldots, n_{\alpha}^{\prime}$ (resp. $\beta, \gamma$ ). In fact, the perturbation of $V_{2}^{\prime} \circ f_{k}$ that we perform near the points of $\mathcal{I}_{k}^{\prime}$ is isotopic through $m$-equivalence to a generic algebraic perturbation of $V_{2}^{\prime} \circ f_{k}$, which itself would yield the usual algebraic braid monodromy invariants as defined by Moishezon and Teicher. 
Still, it seems very unlikely that such pair creation operations are ever needed, and it is reasonable to formulate the following conjecture :

Conjecture. When $X$ is a complex algebraic manifold, the degree doubling formula (19) is valid up to Hurwitz equivalence and global conjugation.

Motivation for this conjecture comes from the following observation. Assume that identifying the braid monodromy given by (19) with that of a generic algebraic map requires the creation of pairs of nodes. Then, considering only the relative motions of the $2 n$ points labelled $1_{\alpha}, \ldots, n_{\alpha}^{\prime}$ (resp. $\beta, \gamma$ ), we obtain two factorizations of $\Delta_{2 n}^{2}$ as a product of positive twists and half-twists in $B_{2 n}$ which are inequivalent in a certain sense. These two factorizations can be thought of as describing the braid monodromy of two symplectic nodal curves in $\mathbb{C P}^{2}$, both irreducible and of identical degree and genus. The braid factorization in $B_{2 n}$ arising from (19) is easily checked to be that of an algebraic nodal curve. Therefore, the inequivalence of the two factorizations would be a strong indication of the possibility of constructing by purely complex algebraic methods a counterexample to the nodal symplectic isotopy conjecture ; this would be extremely surprising.

\section{The Degree Doubling formula for Lefschetz Pencils}

4.1. Braid groups and mapping class groups. We now expand on the ideas in $\S 5$ of [5] to provide a description of the relations between the braid monodromy of a branch curve and the monodromy of the corresponding Lefschetz pencil.

Recall that the Lefschetz pencils determined by approximately holomorphic sections of $L^{\otimes k}$ are obtained from the corresponding branched coverings simply by forgetting one of the three sections, or equivalently by composing the covering map with the projection $\pi: \mathbb{C P}^{2}-\{p t\} \rightarrow \mathbb{C P}^{1}$. In particular the curves making up the pencil are precisely the preimages of the fibers of $\pi$ by the branched covering, and the base points of the pencil are the preimages of the pole of the projection $\pi$.

Consider as previously the branched covering $f_{k}: X \rightarrow \mathbb{C P}^{2}$. Call $n$ its degree and $d$ the degree of its branch curve $D_{k}$, and let $\theta: F_{d}=\pi_{1}\left(\mathbb{C}-\left\{q_{1}, \ldots, q_{d}\right\}\right) \rightarrow S_{n}$ 
be the corresponding geometric monodromy representation. The map $\theta$ determines a simple $n$-fold covering of $\mathbb{C P}^{1}$ branched at $q_{1}, \ldots, q_{d}$; we will denote this covering by $u: \Sigma_{g} \rightarrow \mathbb{C P}^{1}$, where $\Sigma_{g}$ is a Riemann surface of genus $g=1-n+(d / 2)$.

It is important for our purposes to observe that the Riemann surface $\Sigma_{g}$ naturally comes with $n$ marked points, corresponding to the base points of the Lefschetz pencil : these $n$ points are precisely the preimages by $u$ of the point at infinity in $\mathbb{C P}^{1}$. In particular, rather than simply working in the mapping class group $M_{g}$ of $\Sigma_{g}$ in the usual way, we will consider the mapping class group $M_{g, n}$ of a Riemann surface of genus $g$ with $n$ boundary components, i.e. the set of isotopy classes of diffeomorphisms of the complement of $n$ discs centered at the given points in $\Sigma_{g}$ which fix each of the $n$ boundary components (or equivalently, diffeomorphisms of $\Sigma_{g}$ which fix the $n$ marked points and whose tangent map at each of these points is the identity). Describing a Lefschetz pencil by a word in $M_{g, n}$ provides a more complete picture than the usual description using $M_{g}$, as it also accounts for the relative positions of the base points of the pencil with respect to the various vanishing cycles.

Recall the following construction from $[5]:$ let $\mathcal{C}_{n}\left(q_{1}, \ldots, q_{d}\right)$ be the (finite) set of all surjective group homomorphisms $F_{d} \rightarrow S_{n}$ which map each of the geometric generators $\gamma_{1}, \ldots, \gamma_{d}$ of $F_{d}$ to a transposition and map their product $\gamma_{1} \cdots \gamma_{d}$ to the identity element in $S_{n}$. Each element of $\mathcal{C}_{n}\left(q_{1}, \ldots, q_{d}\right)$ determines a simple $n$-fold covering of $\mathbb{C P}^{1}$ branched at $q_{1}, \ldots, q_{d}$.

Denote by $\mathcal{X}_{d}$ the space of configurations of $d$ distinct points in the plane. The set of all simple $n$-fold coverings of $\mathbb{C P}^{1}$ with $d$ branch points and such that no branching occurs above the point at infinity can be thought of as a covering $\tilde{\mathcal{X}}_{d, n}$ above $\mathcal{X}_{d}$, whose fiber above the configuration $\left\{q_{1}, \ldots, q_{d}\right\}$ identifies with $\mathcal{C}_{n}\left(q_{1}, \ldots, q_{d}\right)$. The braid group $B_{d}$ identifies with the fundamental group of $\mathcal{X}_{d}$, and therefore $B_{d}$ acts on the fiber $\mathcal{C}_{n}\left(q_{1}, \ldots, q_{d}\right)$ by deck transformations of the covering $\tilde{\mathcal{X}}_{d, n}$.

Define the subgroup $B_{d}^{0}(\theta)$ as the set of all the loops in $\mathcal{X}_{d}$ whose lift at the point $p_{\theta} \in \tilde{\mathcal{X}}_{d, n}$ corresponding to the covering described by $\theta$ is a closed loop in $\tilde{\mathcal{X}}_{d, n}$, i.e. the set of all braids which act on $F_{d}=\pi_{1}\left(\mathbb{C}-\left\{q_{1}, \ldots, q_{d}\right\}\right)$ in a manner compatible with the covering structure defined by $\theta$. Denoting by $Q_{*}$ the action 
of a braid $Q$ on $F_{d}$, it is easy to check that $B_{d}^{0}(\theta)$ is the set of all braids $Q$ such that $\theta \circ Q_{*}=\theta$.

There exists a natural (tautologically defined) bundle $\mathcal{Y}_{d, n}$ over $\tilde{\mathcal{X}}_{d, n}$ whose fiber is a Riemann surface of genus $g$. Each of these Riemann surfaces comes naturally as a branched covering of $\mathbb{C P}^{1}$, and carries $n$ distinct marked points the preimages of the point at infinity in $\mathbb{C P}^{1}$ by the covering.

Given an element $Q$ of $B_{d}^{0}(\theta) \subset B_{d}$, it can be lifted to $\tilde{\mathcal{X}}_{d, n}$ as a loop based at the point $p_{\theta}$, and the monodromy of the fibration $\mathcal{Y}_{d, n}$ around this loop defines an element of the mapping class group $M_{g, n}$ of a Riemann surface of genus $g$ with $n$ boundary components, which we will call $\theta_{*}(Q)$. More intuitively, viewing $Q$ as a compactly supported diffeomorphism of the plane preserving $\left\{q_{1}, \ldots, q_{d}\right\}$, the fact that $Q \in B_{d}^{0}(\theta)$ means that the diffeomorphism representing $Q$ can be lifted via the covering $u: \Sigma_{g} \rightarrow \mathbb{C P}^{1}$ to a diffeomorphism of $\Sigma_{g}$, whose class in the mapping class group is $\theta_{*}(Q)$.

It is easy to check that the image of the braid monodromy homomorphism is contained in $B_{d}^{0}(\theta)$ : this is because the geometric monodromy representation $\theta$ factors through $\pi_{1}\left(\mathbb{C P}^{2}-D_{k}\right)$, on which the action of the braids arising in the monodromy is clearly trivial. Therefore, we can take the image of the braid factorization by the map $\theta_{*}$ and obtain a factorization in the mapping class group $M_{g, n}$. As observed in [5], all the factors of degree \pm 2 or 3 in the factorization lie in the kernel of $\theta_{*}$; therefore, the only remaining terms are those corresponding to the tangency points of the branch curve $D_{k}$, and each of these is a Dehn twist.

Recall from [5] that the image in the mapping class group $M_{g, n}$ of a half-twist $Q \in B_{d}^{0}(\theta)$ can be constructed as follows. Call $\gamma$ the path in $\mathbb{C}$ joining two of the branch points (say $q_{i}$ and $q_{j}$ ) which describes the half-twist $Q$ ( $\gamma$ is the path along which the twisting occurs). Among the $n$ lifts of $\gamma$ to $\Sigma_{g}$, only two hit the branch points of the covering ; these two lifts have common end points, and together they define a loop $\delta$ in $\Sigma_{g}$. Equivalently, one may also define $\delta$ as one of the two non-trivial lifts of the boundary of a small tubular neighborhood of $\gamma$ in $\mathbb{C}$. In any case, one easily checks that the element $\theta_{*}(Q)$ in $M_{g, n}$ is a positive Dehn twist along the loop $\delta$ (see Proposition 4 of [5]).

As a consequence, one obtains the usual description of the monodromy of the Lefschetz pencil as a word in the mapping class group whose factors are positive 
Dehn twists. However, as observed by Smith in [19], the product of all these Dehn twists is not the identity element in $M_{g, n}$, because after blowing up the pencil at its base points one obtains a Lefschetz fibration in which the exceptional sections have the non-trivial normal bundle $O(-1)$. Instead, the product of all the factors is equal to $\theta_{*}\left(\Delta_{d}^{2}\right)$, which is itself equal to the product of $n$ positive Dehn twists, one along a small loop around each of the $n$ base points of the pencil.

It follows from the above considerations that we can lift the degree doubling formula for braid monodromies obtained in $\S 3$ and obtain a similar formula for Lefschetz pencils. The task is made even easier by the fact that we only need to consider the tangency points of the branch curves.

We now introduce the general setup for the degree doubling formula. To start with, recall that the branch curve $D_{2 k}$ is of degree $\bar{d}=2 d+6 n$, while the degree of the covering $f_{2 k}$ is $4 n$. Recall from $\S 3.4$ the relation between the geometric monodromy factorizations $\theta_{2 k}: F_{\bar{d}} \rightarrow S_{4 n}$ and $\theta_{k}: F_{d} \rightarrow S_{n}:$ as previously, view the $4 n$ sheets of $f_{2 k}$ as four groups of $n$ sheets labelled $i_{a}, i_{b}, i_{c}, i_{d}, 1 \leq i \leq n$, and use the same labelling of the branch points as in $\S 3$. With these notations, the transpositions in $S_{4 n}$ corresponding to the geometric generators around $1, \ldots, d, 1^{\prime}, \ldots, d^{\prime}$ are directly given by the geometric monodromy representation $\theta_{k}$ associated to $D_{k}$ : given $1 \leq r \leq d$, if $\theta_{k}$ maps the $r$-th geometric generator to the transposition $(i j)$ in $S_{n}$ then, calling $\gamma_{r}$ and $\gamma_{r^{\prime}}$ the geometric generators in $F_{\bar{d}}$ corresponding to $r$ and $r^{\prime}$, one gets $\theta_{2 k}\left(\gamma_{r}\right)=\theta_{2 k}\left(\gamma_{r^{\prime}}\right)=\left(i_{a} j_{a}\right)$. Moreover, each of the $n$ copies of $V_{2}$ connects four sheets to each other, one in each group of $n$ : the geometric generators around $i_{\alpha}, i_{\alpha}^{\prime}, i_{\beta}, i_{\beta}^{\prime}, i_{\gamma}$ and $i_{\gamma}^{\prime}$ are mapped by $\theta_{2 k}$ to $\left(i_{a} i_{b}\right),\left(i_{c} i_{d}\right),\left(i_{a} i_{c}\right),\left(i_{b} i_{d}\right),\left(i_{a} i_{d}\right)$ and $\left(i_{b} i_{c}\right)$ respectively, for all $1 \leq i \leq n$.

As a consequence, $\theta_{2 k}$ determines a $4 n$-fold branched covering $\bar{u}: \Sigma_{\bar{g}} \rightarrow \mathbb{C P}^{1}$, with $\bar{g}=2 g+n-1$, whose structure is as follows. First, the preimage of a disc $\mathcal{D}$ containing the $d$ points labelled $1, \ldots, d$ consists of $3 n+1$ components. One of these components (the sheets $1_{a}, \ldots, n_{a}$ ) is a $n$-fold covering identical to the one described by $\theta_{k}$, i.e. it naturally identifies with the fiber $\Sigma_{g}$ of the Lefschetz pencil associated to $f_{k}$, with $n$ small discs removed. These punctures correspond to the preimages of a small disc around the point at infinity in the covering $u: \Sigma_{g} \rightarrow \mathbb{C P}^{1}$, i.e. they correspond to small discs around the base points in $\Sigma_{g}$. The other $3 n$ components of $\bar{u}^{-1}(\mathcal{D})$, in which no branching occurs, are topologically trivial. 
The same picture also describes the preimage of a disc $\mathcal{D}^{\prime}$ containing the $d$ points labelled $1^{\prime}, \ldots, d^{\prime}$ : there is one non-trivial component which can be identified with $\Sigma_{g}$ punctured at its base points, and the other $3 n$ components are just plain discs.

Finally, the preimage by $\bar{u}$ of the cylinder $\mathbb{C P}^{1}-\left(\mathcal{D} \cup \mathcal{D}^{\prime}\right)$ consists of $n$ components, each of which is a four-sheeted covering branched at six points, i.e. topologically a sphere with eight punctures. Actually, each of these $n$ components may be thought of as the fiber of the Lefschetz pencil corresponding to the covering $V_{2}$ (since we restrict ourselves to a cylinder we get eight punctures). For each $i \in\{1, \ldots, n\}$ the corresponding component of $\bar{u}^{-1}\left(\mathbb{C P}^{1}-\left(\mathcal{D} \cup \mathcal{D}^{\prime}\right)\right)$ connects together the non-trivial components of $\bar{u}^{-1}(\mathcal{D})$ and $\bar{u}^{-1}\left(\mathcal{D}^{\prime}\right)$ with the trivial components corresponding to the sheets $i_{b}, i_{c}$ and $i_{d}$.

In the end the Riemann surface $\Sigma_{\bar{g}}$ can be thought of as two copies of $\Sigma_{g}$ glued together at the $n$ base points. This description coincides exactly with the one obtained by Smith in [19] via more direct methods.

4.2. The degree doubling formula for Lefschetz pencils. In order to simplify the description of the degree doubling formula for Lefschetz pencils, we want to slightly modify the setup of $\S 3$.

First, we want to choose a different picture for $\theta_{k}$ : recall that global conjugations in $B_{d}$ make it possible to choose the most convenient geometric monodromy representation $\theta_{k}: F_{d} \rightarrow S_{n}$. As a consequence we chose in $\S 3$ a setup that made the final answer (19) relatively easy to express, but as observed in the remark at the end of $\S 3.6$ we could just as well have worked with any other choice of $\theta_{k}$, the only price being a slightly more complicated expression for the degree doubling formula. Note that the change of $\theta_{k}$ only affects factors of degree \pm 2 in the formula, and therefore the half-twists which are relevant for our purposes are not affected.

Here we want to choose $\theta_{k}$ in such a way that the $i$-th geometric generator $\gamma_{i}$ is mapped to the transposition $(1,2)$ if $i \leq d-2(n-1)=2 g$, and $\theta_{k}\left(\gamma_{d-2 j}\right)=$ $\theta\left(\gamma_{d-2 j-1}\right)=(n-j-1, n-j)$ for all $j \leq n-2$. In other words, the transpositions $\theta_{k}\left(\gamma_{i}\right)$ correspond to the factorization

$$
\mathrm{Id}=(1,2)^{2 g} \cdot \prod_{i=1}^{n-1}(i, i+1)^{2}
$$


in $S_{n}$. Another change that we want to make is in the ordering of the $\bar{d}=2 d+6 n$ points that appear in the diagrams of $\S 3$ along the real axis. Namely, we want to replace the ordering $1, \ldots, d, 1^{\prime}, \ldots, d^{\prime}, 1_{\alpha}, \ldots, n_{\gamma}^{\prime}$ used in $\S 3$ by the new ordering $1, \ldots, d, 1_{\alpha}, \ldots, n_{\gamma}^{\prime}, d^{\prime}, \ldots, 1^{\prime}$. This is done by first moving the $d$ points $1^{\prime}, \ldots, d^{\prime}$ clockwise around the points $1_{\alpha}, \ldots, n_{\gamma}^{\prime}$ by a half-turn, and then by rotating a disc containing the $d$ points $1^{\prime}, \ldots, d^{\prime}$ counterclockwise by a half-turn.

Finally, in order to better visualize the positions of the base points of the pencil (the $4 n$ marked points on $\Sigma_{\bar{g}}$ ), we want to move the fiber in which they lie from the point at infinity in $\mathbb{C P}^{1}$ back into our picture. We choose to move the base points so that they correspond to the preimages of a point $b$ on the real axis lying inbetween the point labelled $d$ and the point labelled $1_{\alpha}$. The motion bringing the point at infinity to $b$ is performed along a vertical line in the upper half-plane (this motion of course affects some of the braids, but it was chosen in such a way that the resulting changes are minimal).

The effect of all these changes is to make the covering $\bar{u}: \Sigma_{\bar{g}} \rightarrow \mathbb{C P}^{1}$ easier to visualize, while simplifying the paths corresponding to the half-twists in (19). The picture is the following :

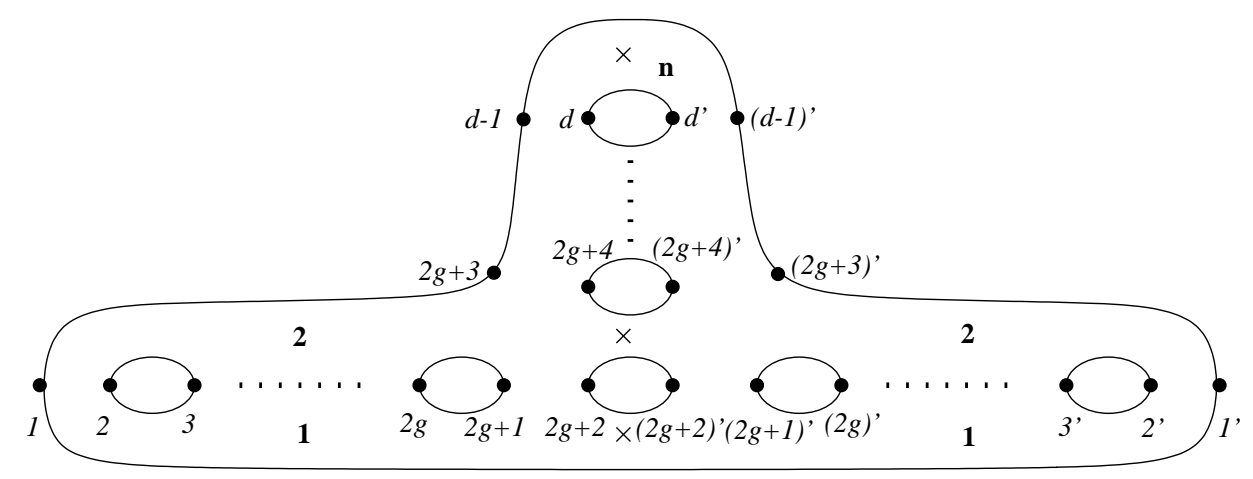

In this picture, the labels in italics correspond to branch points and those in boldface correspond to the sheets of the covering ; for simplicity we have omitted the branch points $1_{\alpha}, \ldots, n_{\gamma}^{\prime}$, which should be placed in the necks joining the two halves, and the $3 n$ other sheets which do not contribute to the topology. When the $3 n$ sheets $1_{b}, \ldots, n_{d}$ are collapsed, the corresponding base points are brought back to the sheets $1_{a}, \ldots, n_{a}$ near the branch points $1_{\alpha}, \ldots, n_{\gamma}^{\prime}$; therefore, on the picture each $\times$ mark corresponds to four base points. 
In order to understand the Lefschetz pencil corresponding to $f_{2 k}$, we need to place the various half-twists appearing in the braid factorization of $D_{2 k}$ on this picture. A first set of half-twists comes from the braid factorization of $D_{k}$. These half-twists correspond exactly to the Dehn twists appearing in monodromy of the Lefschetz pencil for $f_{k}$, after a suitable embedding of $M_{g, n}$ into the mapping class group $M_{\bar{g}, 4 n}$. Recall that the braid factorization in $B_{d}$ corresponding to $D_{k}$ is embedded into $B_{\bar{d}}$ by considering a disc $\mathcal{D}$ containing the $d$ points labelled $1, \ldots, d$. Therefore, the corresponding embedding of the mapping class group $M_{g, n}$ into the larger mapping class group $M_{\bar{g}, 4 n}$ is geometrically realized by the embedding into $\Sigma_{\bar{g}}$ of the main connected component of $\bar{u}^{-1}(\mathcal{D})$, which as we know from $\S 4.1$ naturally identifies with the Riemann surface $\Sigma_{g}$ punctured at each of the $n$ base points. On the above picture of $\Sigma_{\bar{g}}$ this corresponds to the left half of the diagram.

Observe that all the other half-twists appearing in the braid factorization for $D_{2 k}$ are completely standard and depend only on $d$ and $n$ rather than on the actual topology of the manifold $X$. Therefore, the degree doubling formula for Lefschetz pencils is once again a universal formula : the word in $M_{\bar{g}, 4 n}$ describing the Lefschetz pencil in degree $2 k$ is obtained by embedding the word describing the pencil in degree $k$ via the above-described map from $M_{g, n}$ into $M_{\bar{g}, 4 n}$ and adding to it a completely standard set of Dehn twists which depends only on $g$ and $n$ but not on the actual topology of the manifold $X$. This observation was already made by Ivan Smith in [19].

The extra half-twists appearing in the degree doubling formula for braid monodromies are $\check{Z}_{i i^{\prime}}$ and $\hat{Z}_{i i^{\prime}}$ for $1 \leq i \leq d$, and $\tilde{Z}_{i_{\alpha} i_{\alpha}^{\prime} ;\left(i_{\beta} i_{\beta}^{\prime}\right)}, \tilde{Z}_{i_{\alpha} i_{\alpha}^{\prime} ;\left(i_{\gamma} i_{\gamma}^{\prime}\right)}$ and $\tilde{Z}_{i_{\beta} i_{\beta}^{\prime} ;\left(i_{\gamma} i_{\gamma}^{\prime}\right)}$ for $1 \leq i \leq n$, as described in $\S 3.6$ (their total number $2 d+3 n$ is in agreement with an easy calculation of Euler-Poincaré characteristics). We will now describe the Dehn twists corresponding to these half-twists.

After the global conjugation described above, $\check{Z}_{i i^{\prime}}$ becomes a half-twist along the following path :

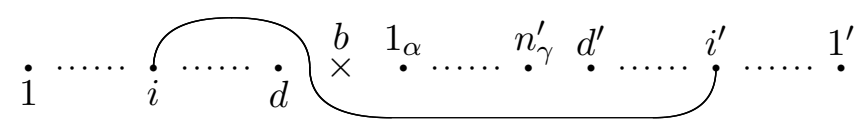


Its lift to the mapping class group $M_{\bar{g}, 4 n}$ is a Dehn twist that we will call $\check{\tau}_{i}$, and which can be represented as follows when $i$ is even and $i \leq 2 g$ :

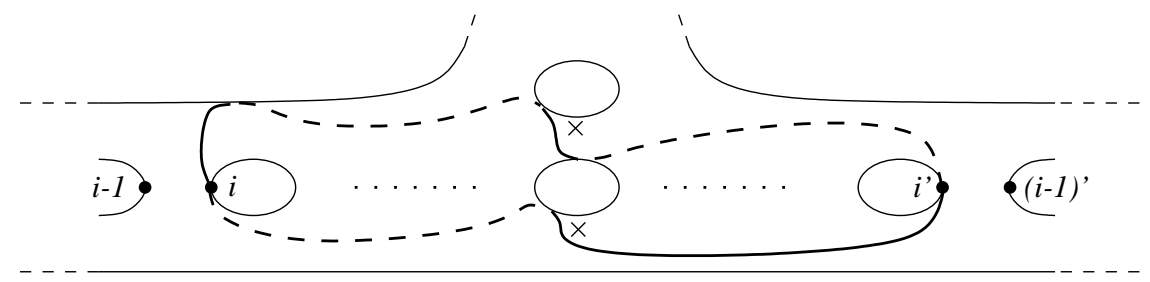

For $i$ odd and $i \leq 2 g+1$, the picture describing $\check{\tau}_{i}$ becomes the following :

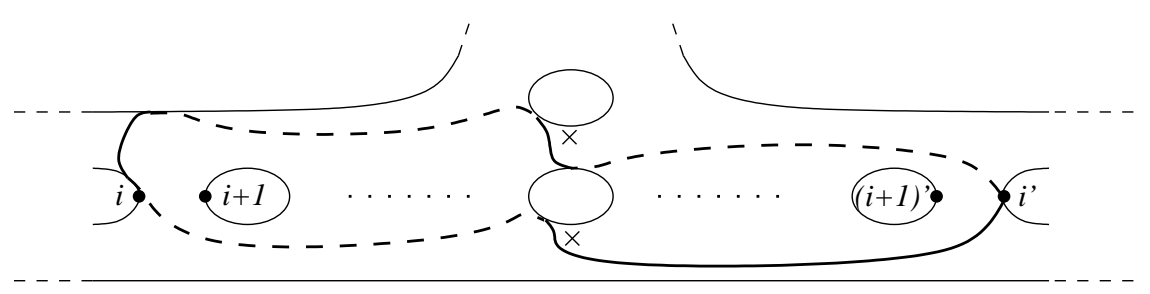

When $i=1$ the undrawn parts on both sides of the picture are just discs and the picture can therefore be slightly simplified ; conversely, when $i=2 g+1$ the points labelled $(i+1)$ and $(i+1)^{\prime}$ are immediately on both sides of the central neck rather than as pictured.

For $i$ even and $i \geq 2 g+2, \check{\tau}_{i}$ is described by the following picture (the two necks shown correspond to the sheets numbered $s$ and $s+1$, where $\left.s=\frac{1}{2}(i-2 g) \geq 1\right)$ :

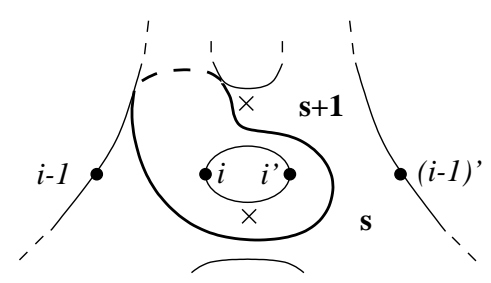

Finally, when $i$ is odd and $i \geq 2 g+3$, the picture describing $\check{\tau}_{i}$ becomes the following (the two necks shown correspond to the sheets numbered $s$ and $s+1$, where $\left.s=\frac{1}{2}(i+1-2 g) \geq 2\right)$ : 


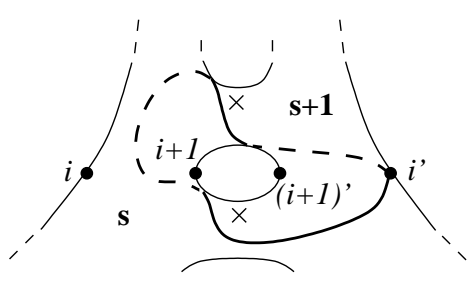

We now turn to $\hat{Z}_{i i^{\prime}}$, which after the above-described global conjugation becomes a half-twist along the following path :

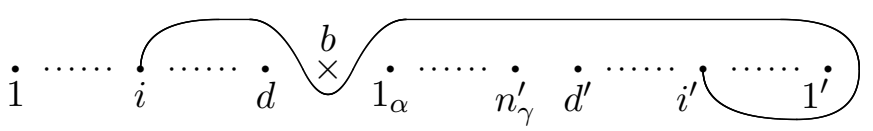

This path can be homotoped into the following one, which goes through the point at infinity in $\mathbb{C P}^{1}$ :

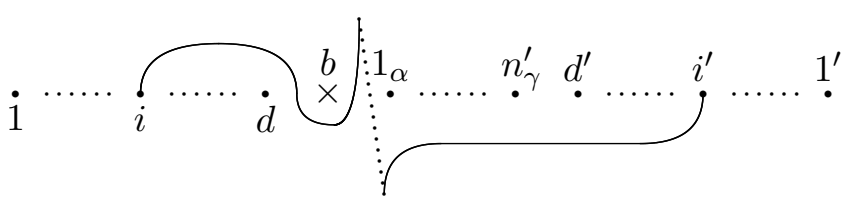

Therefore, the Dehn twists $\hat{\tau}_{i} \in M_{\bar{g}, 4 n}$ obtained by lifting $\hat{Z}_{i i^{\prime}}$ only differ from $\check{\tau}_{i}$ by a twisting in each of the necks joining the two halves of $\Sigma_{\bar{g}}$. As a result, we get the following pictures (using the same notations as for $\check{\tau}_{i}$ ) :
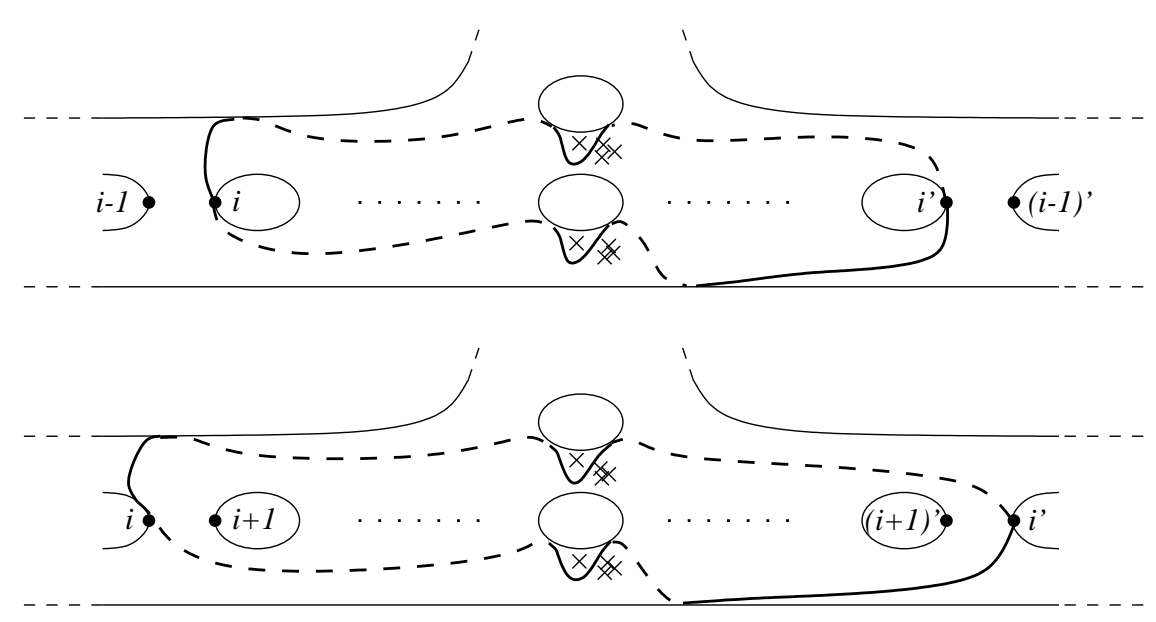
The first picture corresponds to the case $i$ even, $i \leq 2 g$; the second one to $i$ odd, $i \leq 2 g+1$. In each of the two necks, the vanishing loop circles around the base point corresponding to the sheet labelled $1_{a}$ (resp. $2_{a}$ ), but not around those corresponding to sheets $1_{b}, 1_{c}$ and $1_{d}$ (resp. $2_{b}, 2_{c}, 2_{d}$ ). When $i \geq 2 g+2$, the pictures become the following (the left one is for even $i$, the right one for odd $i$ ) :
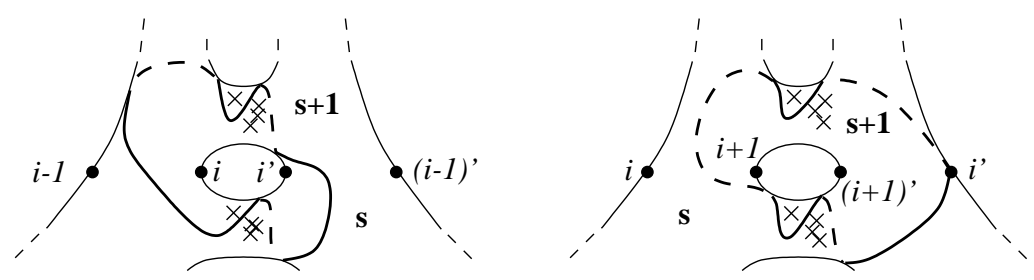

We now turn to the half-twists $\tilde{Z}_{i_{\alpha} i_{\alpha}^{\prime} ;\left(i_{\beta} i_{\beta}^{\prime}\right)}, \tilde{Z}_{i_{\alpha} i_{\alpha}^{\prime} ;\left(i_{\gamma} i_{\gamma}^{\prime}\right)}$ and $\tilde{Z}_{i_{\beta} i_{\beta}^{\prime} ;\left(i_{\gamma} i_{\gamma}^{\prime}\right)}(1 \leq i \leq$ $n)$. To simplify the diagrams we only represent the relevant points, i.e. we forget $j_{\alpha}, j_{\alpha}^{\prime}, j_{\beta}, j_{\beta}^{\prime}, j_{\gamma}, j_{\gamma}^{\prime}$ for $j \neq i$ as these points do not play any role. Moreover, we use the observation that, for the purposes of computing the corresponding Dehn twists, we are allowed to move a path across a branch point if the corresponding sheets of the covering are distinct. Finally, we further simplify the diagrams by allowing ourselves to draw paths which go through the point at infinity in $\mathbb{C P}^{1}$. With all these simplifications, we get the following diagrams :

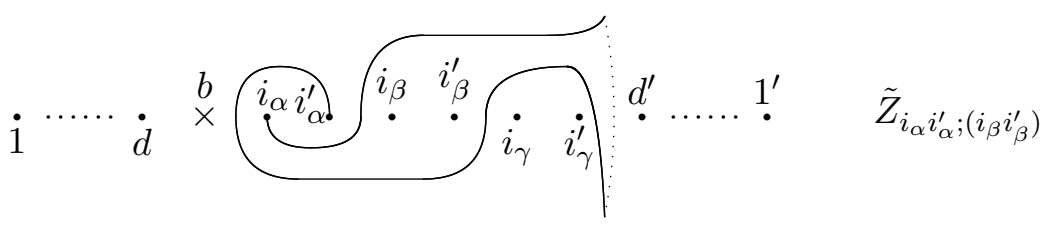

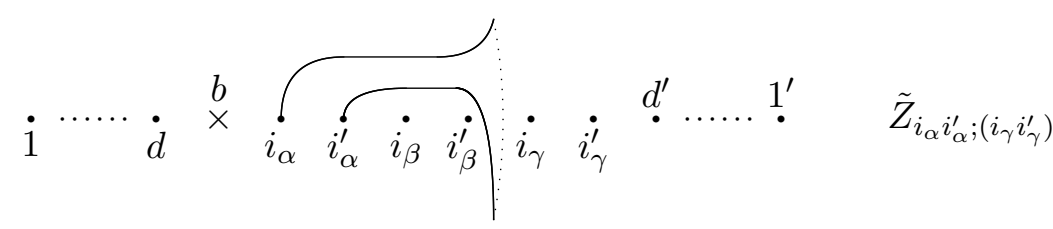

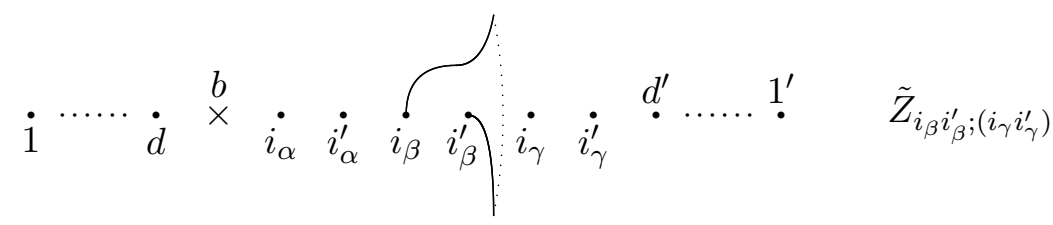


It is now clear that the only relevant parts of $\Sigma_{\bar{g}}$ are the sheets labelled $i_{b}, i_{c}$, $i_{d}$ of the covering, as well as the part of the sheet labelled $i_{a}$ that lies inbetween the points $1, \ldots, d$ and $d^{\prime}, \ldots, 1^{\prime}$. In particular, the loops we obtain are entirely located in the $i$-th neck joining the two halves of $\Sigma_{\bar{g}}$; if we forget about the base points, the Dehn twists $\tau_{i, \alpha \beta}, \tau_{i, \alpha \gamma}$ and $\tau_{i, \beta \gamma}$ corresponding to the half-twists $\tilde{Z}_{i_{\alpha} i_{\alpha}^{\prime} ;\left(i_{\beta} i_{\beta}^{\prime}\right)}, \tilde{Z}_{i_{\alpha} i_{\alpha}^{\prime} ;\left(i_{\gamma} i_{\gamma}^{\prime}\right)}$ and $\tilde{Z}_{i_{\beta} i_{\beta}^{\prime} ;\left(i_{\gamma} i_{\gamma}^{\prime}\right)}$ are equal to each other, and are twists along a loop that simply goes around the $i$-th neck joining the two halves of $\Sigma_{\bar{g}}$.

In the presence of the four base points lying in the sheets $i_{a}, i_{b}, i_{c}$ and $i_{d}$ of the covering, we have to be more careful, but it can be checked that the Dehn twists $\tau_{i, \alpha \beta}, \tau_{i, \alpha \gamma}$ and $\tau_{i, \beta \gamma}$ are respectively given by the following diagrams (only the $i$-th neck is shown; the base points are labelled $a, b, c$, and $d)$ :

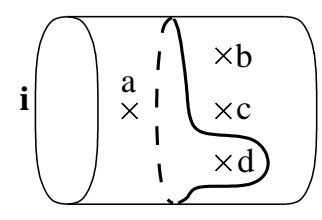

$\tau_{i, \alpha \beta}$

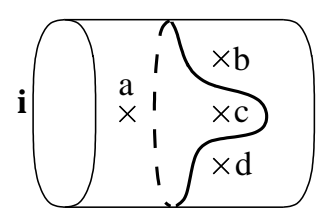

$\tau_{i, \alpha \gamma}$

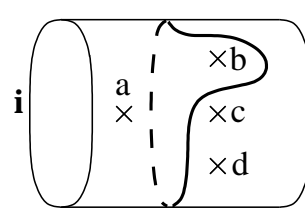

$\tau_{i, \beta \gamma}$

Summarizing, we get the following result :

Theorem 4. Let $X$ be a compact symplectic 4-manifold, and consider the structure of symplectic Lefschetz pencil on $X$ given by two sections of $L^{\otimes k}$. Let $g$ be the genus of the fiber $\Sigma_{g}$, and let $n$ be the number of base points. Let $d=2 g-2+2 n$, and call $\Psi_{g}$ the word in the mapping class group $M_{g, n}$ describing the monodromy of this pencil.

Let $\bar{g}=2 g+n-1$, and view a Riemann surface $\Sigma_{\bar{g}}$ of genus $\bar{g}$ as obtained by gluing together two copies of $\Sigma_{g}$ at the base points. Call $\iota: M_{g, n} \rightarrow M_{\bar{g}, 4 n}$ the inclusion map discussed above.

Then, provided that $k$ is large enough and using the notations described above, the monodromy of the symplectic Lefschetz pencil structure obtained on $X$ from sections of $L^{\otimes 2 k}$ is given by the word $\Psi_{\bar{g}}$ in the mapping class group $M_{\bar{g}, 4 n}$, where

$$
\Psi_{\bar{g}}=\prod_{i=1}^{d} \check{\tau}_{i} \cdot \prod_{i=1}^{d} \hat{\tau}_{i} \cdot \iota\left(\Psi_{g}\right) \cdot \prod_{i=1}^{n} \tau_{i, \alpha \beta} \cdot \prod_{i=1}^{n} \tau_{i, \alpha \gamma} \cdot \prod_{i=1}^{n} \tau_{i, \beta \gamma},
$$

and the Dehn twists $\check{\tau}_{i}, \hat{\tau}_{i}, \tau_{i, \alpha \beta}, \tau_{i, \alpha \gamma}$ and $\tau_{i, \beta \gamma}$ are as described above. 
Remark. One must be aware of the fact that, in the formula (20), composition products are written from left to right. This convention, which is the usual one for braid groups, is the opposite of the usual notation for composition products when working with diffeomorphisms (the order of the factors then needs to be reversed).

It is also worth observing that the product of the factors in $\iota\left(\Psi_{g}\right)$ is almost exactly the twist by which $\hat{\tau}_{i}$ differs from $\check{\tau}_{i}$, the only difference being in the position of the base points with respect to the vanishing cycle. Therefore, if we forget about the base points, a sequence of Hurwitz moves in (20) yields the following slightly simpler formula (in $M_{\bar{g}, 0}$ instead of $M_{\bar{g}, 4 n}$, and observing that $\tau_{i, \alpha \beta}, \tau_{i, \alpha \gamma}$ and $\tau_{i, \beta \gamma}$ are equal in $\left.M_{\bar{g}, 0}\right)$ :

$$
\Psi_{\bar{g}}=\prod_{i=1}^{d} \check{\tau}_{i} \cdot \iota\left(\Psi_{g}\right) \cdot \prod_{i=1}^{d} \check{\tau}_{i} \cdot \prod_{i=1}^{n} \tau_{i, \alpha \beta}^{3} .
$$

This expression contains many pairs of identical factors; by a result of Seidel (cf. section (16g) of [18], see also $\S 8$ of [7]), this means that the Lefschetz fibration with total space a blow-up of $X$ and monodromy $\Psi_{\bar{g}}$ contains many Lagrangian $(-2)$ spheres, obtained by connecting pairs of isotopic vanishing cycles (among those introduced by the degree doubling procedure). However these spheres collapse when the Lefschetz fibration is blown down along its exceptional sections, as they intersect non-trivially two such sections.

The correctness of the formula (20) can be checked easily in some simple examples : for instance, a generic pencil of conics on $\mathbb{C P}^{2}$ has three singular fibers, and can be considered as obtained from a pencil of lines by the procedure described above. This corresponds to the limit case where $n=1, d=0, g=0$ and the word $\Psi_{g}$ is empty. The three Dehn twists $\tau_{1, \alpha \beta}, \tau_{1, \alpha \gamma}$ and $\tau_{1, \beta \gamma}$ in $M_{0,4}$ then coincide with the well-known picture.

Another simple example that can be considered is the case of a pencil of curves of degree $(1,1)$ on $\mathbb{C P}^{1} \times \mathbb{C P}^{1}$. The generic fiber of this pencil is a rational curve $(d=2, n=2, g=0)$, and there are two singular fibers. The corresponding word in $M_{0,2}$ is $\tau \cdot \tau$, where $\tau$ is a positive Dehn twist along a simple curve separating the two base points. The degree doubling procedure yields a word in $M_{1,8}$ consisting of 12 Dehn twists. Forgetting the positions of the base points, one easily checks that the reduction of this word to $M_{1,0} \simeq S L(2, \mathbb{Z})$ is Hurwitz equivalent to the 
well-known monodromy of the elliptic surface $E(1)$, which is exactly what one obtains by blowing up the eight base points of a pencil of curves of degree $(2,2)$ on $\mathbb{C P}^{1} \times \mathbb{C P}^{1}$.

\section{REFERENCES}

[1] D. Auroux, Symplectic 4-manifolds as Branched Coverings of $\mathbb{C P}^{2}$, Inventiones Math. 139 (2000), 551-602.

[2] D. Auroux, Symplectic maps to projective spaces and symplectic invariants, Proc. 7th Gökova Geometry-Topology Conference (2000), International Press, 2001, 1-42 (math.GT/0007130).

[3] D. Auroux, Estimated transversality in symplectic geometry and projective maps, in "Symplectic Geometry and Mirror Symmetry", Proc. 4th KIAS International Conference, Seoul (2000), World Scientific, 2001, 1-30 (math.SG/0010052).

[4] D. Auroux, S. K. Donaldson, L. Katzarkov, M. Yotov, Fundamental groups of complements of plane curves and symplectic invariants, Topology 43 (2004), 1285-1318 (math.GT/0203183).

[5] D. Auroux, L. Katzarkov, Branched Coverings of $\mathbb{C P}^{2}$ and Invariants of Symplectic 4manifolds, Inventiones Math. 142 (2000), 631-673.

[6] D. Auroux, V. S. Kulikov, V. V. Shevchishin, Regular homotopy of Hurwitz curves, Izv. Math. 68 (2004), 521-542 (math.SG/0401172).

[7] D. Auroux, V. Muñoz, F. Presas, Lagrangian submanifolds and Lefschetz pencils, J. Symplectic Geom. 3 (2005), 171-219.

[8] S.K. Donaldson, Symplectic Submanifolds and Almost-complex Geometry, J. Differential Geom. 44 (1996), 666-705.

[9] S.K. Donaldson, Lefschetz Fibrations in Symplectic Geometry, Documenta Math., Extra Volume ICM 1998, II, 309-314.

[10] S.K. Donaldson, Lefschetz Pencils on Symplectic Manifolds, J. Diff. Geom. 53 (1999), 205236.

[11] E. Liberman, M. Teicher, The Hurwitz equivalence problem is undecidable, preprint (math.LO/0511153).

[12] B. Moishezon, Stable Branch Curves and Braid Monodromies, Algebraic Geometry (Chicago, 1980), Lecture Notes in Math. 862, Springer, 1981, 107-192.

[13] B. Moishezon, On cuspidal branch curves, J. Algebraic Geom. 2 (1993), 309-384.

[14] B. Moishezon, The Arithmetic of Braids and a Statement of Chisini, Geometric Topology (Haifa, 1992), Contemp. Math. 164, Amer. Math. Soc., Providence, 1994, 151-175.

[15] B. Moishezon, Topology of Generic Polynomial Maps in Complex Dimension Two, preprint.

[16] B. Moishezon, M. Teicher, Braid Group Technique in Complex Geometry V. The fundamental group of a complement of a branch curve of a Veronese generic projection, Comm. Anal. Geom. 4 (1996), 1-120. 
[17] P. Seidel, Vanishing cycles and mutation, Proc. 3rd European Congress of Mathematics (Barcelona, 2000), Vol. II, Progr. Math. 202, Birkhäuser, Basel, 2001, pp. 65-85 (math.SG/0007115).

[18] P. Seidel, Fukaya categories and Picard-Lefschetz theory, to appear in ETH Lecture Notes series, European Math. Soc.

[19] I. Smith, Lefschetz Fibrations and Divisors in Moduli Space, Geom. Topol. 5 (2001), 579608.

[20] M. Teicher, Braid groups, algebraic surfaces and fundamental groups of complements of branch curves, Algebraic Geometry (Santa Cruz, 1995), Proc. Sympos. Pure Math., 62 (part 1), Amer. Math. Soc., 1997, 127-150.

Denis Auroux

Department of Mathematics

M.I.T., Cambridge, MA 02139

E-mail: auroux@math.mit.edu

Ludmil Katzarkov

Department of Mathematics

University of Miami, Coral Gables, FL33124

E-mail: 1.katzark@math.miami.edu 